\title{
WATER QUALITY MODELING: A REVIEW OF THE ANALYSIS OF UNCERTAINTY
}

\author{
M. Bruce Beck \\ Imperial College of Science and Technology \\ London, UK
}

RR-88-3

April 1988

Reprinted from Water Resources Research, volume 23, No. 8, August 1987.

INTERNATIONAL INSTITUTE FOR APPLIED SYSTEMS ANALYSIS

Laxenburg, Austria 
Research Reports, which record research conducted at IIASA, are independently reviewed before publication. However, the views and opinions they express are not necessarily those of the Institute or the National Member Organizations that support it.

Reprinted with permission from Water Resources Research, 23(8), August 1987, 1393-1442.

Copyright (C) 1987, American Geophysical Union (Washington, DC).

All rights reserved. No part of this publication may be reproduced or transmitted in any form or by any means, electronic or mechanical, including photocopy, recording, or any information storage or retrieval system, without permission in writing from the copyright holder.

Printed by Novographic, Vienna, Austria 


\section{FOREWORD}

Problems of water quality management and protection have been the subject of continuous interest in IIASA research activities for many years, because of their importance both for science and practice. There have been different ways in which these problems have been explored and studied in the framework of various research projects conducted here, but one thing cannot be questioned: the need for deep understanding of phenomena and mathematical methods used to describe them.

The paper by M.B. Beck, an IIASA alumnus, addresses very important issues of uncertainty in water quality modeling. However, the issue of uncertainty is important not only for those who are interested in developing or using water quality models, but also for a wide audience of researchers involved in environmental modeling. Although Beck discusses issues which are not investigated in the framework of the project Decision Support Systems for Managing Large International Rivers, this does not mean that the problems discussed in the paper are irrelevant to the scope of the project. His comprehensive review provides interesting and important information and may stimulate a critical evaluation of the concepts and opinions presented.

K.A. SALEWICZ

Project Leader

Large International Rivers 


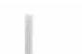




\title{
Water Quality Modeling: A Review of the Analysis of Uncertainty
}

\author{
M. B. BECK
}

\author{
Department of Civil Engineering, Imperial College, London, England
}

\begin{abstract}
This paper reviews the role of uncertainty in the identification of mathematical models of water quality and in the application of these models to problems of prediction. More specifically, four problem areas are examined in detail: uncertainty about model structure, uncertainty in the estimated model parameter values, the propagation of prediction errors, and the design of experiments in order to reduce the critical uncertainties associated with a model. The review is rather lengthy, and it has therefore been prepared in effect as two papers. There is a shorter, largely nontechnical version, which gives a quick impression of the current and future issues in the analysis of uncertainty in water quality modeling. Enclosed by this shorter discussion is the main body of the review dealing in turn with (1) identifiability and experimental design, (2) the generation of preliminary model hypotheses under conditions of sparse, grossly uncertain field data, (3) the selection and evaluation of model structure, (4) parameter estimation (model calibration), (5) checks and balances on the identified model, i.e., model "verification" and model discrimination, and (6) prediction error propagation. Much time is spent in discussing the algorithms of system identification, in particular, the methods of recursive estimation, and in relating these algorithms and the subject of identification to the problems of prediction uncertainty and first-order error analysis. There are two obvious omissions from the review. It is not concerned primarily with either the development and solution of stochastic differential equations or the issue of decision making under uncertainty, although clearly some reference must be made to these topics. In brief, the review concludes (not surprisingly) that much work has been done on the analysis of uncertainty in the development of mathematical models of water quality, and much remains to be done. A lack of model identifiability has been an outstanding difficulty in the interpretation and explanation of past observed system behavior, and there is ample evidence to show that the "larger," more "comprehensive" models are easily capable of generating highly uncertain predictions of future behavior. For the future of the subject, it is speculated that there is the possibility of progress in the development of novel algorithms for model structure identification, a need for new questions to be posed in the problem of prediction, and a distinct challenge to the conventional views of this review in the new forms of knowledge representation and manipulation now emerging from the field of artificial intelligence.
\end{abstract}

\section{CONTENTS}

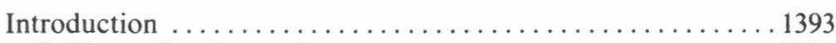
Guidance for the reader $\ldots \ldots \ldots \ldots \ldots \ldots \ldots \ldots \ldots \ldots . \ldots \ldots$

The issues ...................................... 1394

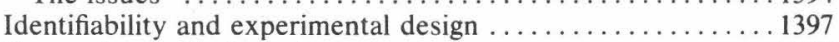

Generating preliminary hypotheses ...................... 1399

The Hornberger-Spear-Young algorithm ............... 1399

Case studies .................................... 1400

Commentary .................................1400

Selection and evaluation of model structure $\ldots \ldots \ldots \ldots \ldots 1401$

Definitions of related problems ....................1401

Recursive and batch estimation algorithms ............ 1402

Uncertainty and state-parameter estimation ...........1402

Model structure identification: an organizing principle .....1405

Case studies ................................... 1407

Commentary ...................................

Parameter estimation ............................... 1412

Types of estimator ......................... 1412

Algorithms for the implementation of batch estimation

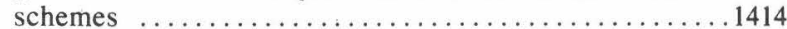

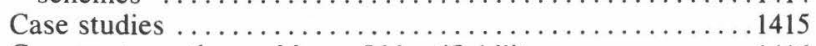

Commentary: the problem of identifiability $\ldots \ldots \ldots \ldots \ldots 1416$

Prudent transformations of ill-posed problems ...........1419

Checks and balances ...............................

Analysis of the residual errors of mismatch . . . . . . . . . 1421

Model discrimination .......................... 1421

Commentary .....................................1421

Prediction error propagation $\ldots \ldots \ldots \ldots \ldots \ldots \ldots \ldots \ldots 1422$

Prediction after identification ..................... 1422

Alternative conceptual frameworks ................. 1423

Approaches and methods ........................1424

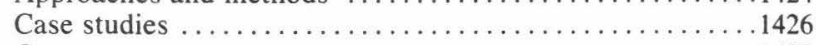

Commentary ................................

Copyright 1987 by the American Geophysical Union.

Paper number 6W0756.

0043-1397/87/006W-0756\$05.00

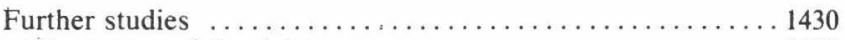

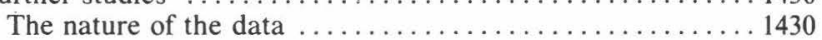

Failure .................................. 1431

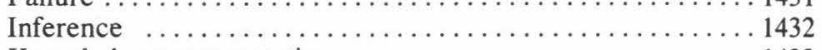

Knowledge representation ..................... 1433

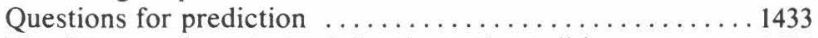

The future under substantially changed conditions ....... 1434

Decision making under uncertainty . . . . . . . . . . . . 1435

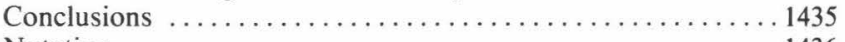

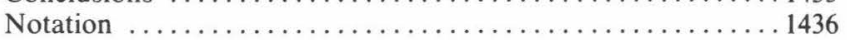

\section{INTRODUCTION}

Uncertainty is such a pervasive, common aspect of experience that a review of its implications for water quality modeling might seem redundant or a statement of the obvious. The present emphasis on uncertainty and error analysis in water quality modeling, which first appears to have been directly addressed by O'Neill [1973] in relation to ecological systems more generally, must at least partly be seen as a reaction to the absence of considerations of uncertainty in the mainstream developments of the subject during the 1960's and early 1970's. If there were a longer term view to be taken, current research activities might be interpreted as a swing of the pendulum away from determinism toward indeterminism. That indeterminism may itself become unfashionable is therefore to be expected. Brush [1980], for example, in discussing the philosophical implications of quantum mechanics and Heisenberg's principle of indeterminacy, postulates just such a cyclical oscillation in "world views," which he traces well back into the nineteenth century.

Why then, more specifically, has the analysis of uncertainty become so important, and what are the particular problems it 
poses? Its importance is partly a reflection of the process of maturation typical of any subject of research. It is partly too a consequence of the liberating influence of the growth in the speed and capacity of digital computing equipment. The difficulties of mathematical modeling are not now questions of whether the equations can be solved and of the costs of solving them many times; nor are they essentially questions of whether prior theory (on transport, dispersion, growth, decay, predation, etc.) is potentially capable of describing the system's behavior. The important questions are those of whether prior theory adequately matches observed behavior and whether the predictions obtained from models are meaningful and useful.

The scope and purpose of this review are accordingly to survey, classify, and evaluate the methods that have been developed and applied to analysis of the following four problem areas associated with uncertainty.

Problem area $1(P 1)$. Uncertainty about the relationships among the variables characterizing the dynamic behavior of systems, i.e., uncertainty about model structure.

Problem area $2(P 2)$. Uncertainty about the value of the parameters (coefficients) appearing in the identified structure of the dynamic model for the system's behavior.

Problem area $3(P 3)$. Uncertainty associated with predictions of the future behavior of the system.

Problem area 4 (P4). The design of experiments, or monitoring programs, for the specific purpose of reducing the critical uncertainties associated with a model.

\subsection{Guidance for the Reader}

When confronted with a paper of this length, the attention of the casual reader is not easily engaged; indeed, some may not even have persevered this far. But the review is composed of two papers. There is a shorter, largely nontechnical version for those who wish merely to acquire a quick impression of the current and future issues associated with uncertainty. This comprises sections 1,8 , and 9 ; it is a philosophical comment on the status quo and a speculative view of future developments of the subject. There is also the paper as a whole, which is therefore the longer version of the review for those with a determined interest in detail. Among this considerable detail, the reader who is most familiar with the long-standing problem of model calibration may wish merely to read section 5 , which is more or less self-contained for this purpose.

The paper has a certain symmetry about it. We shall begin shortly in this section with questions of management and decision making and then transfer quickly to questions of a more detailed scientific nature. When the shorter version of the paper is resumed in section 8 , it continues with a discussion of essentially scientific issues but moves finally to matters of decision-making uncertainty. This will clearly not satisfy the reader whose primary concerns are the more practical problems of managing water quality, and that reader might therefore prefer to read a (equally lengthy) review of these topics presented recently elsewhere [Beck, 1985a].

The longer version of the paper is also symmetrical in that it reflects the cyclical nature of the problems: from experimental design (in section 2) through identification, to prediction, and back again to experimental design (at the end of section 7). Its connection with section 8 is precisely on this point of experimental design, and in fact, section 8 once again runs through the same cycle in its brief examination of the likely problems of the future.

Each section of the main body of the paper (sections 2-7) is organized as follows. It begins with a brief statement of the logic behind the position of the topic in the overall discussion. There is then a definition of the problem, followed by a description of the approaches and, where appropriate, the specification of certain important algorithms. The relevant case study results are then surveyed, and finally some critical comments are made on the notable successes and outstanding problems. Section 8 is thus a response to many of these outstanding problems. Some of the methods presented are relevant to more than one section, and some of the sections could easily be overwhelmed by a concentration of methodological detail. To provide a better balance therefore, and a more easily readable style, section 4 introduces the recursive methods of state-parameter estimation (these methods also being relevant to sections 5 and 7), and section 5 is complementary in its introduction of batch methods of estimation.

The scope of the review may be further qualified by defining the relevant field of water quality: the focus will be on water quality in surface freshwater systems, where this includes topics from the related but more general field of systems ecology. Despite this, however, the reader should be aware of the rich literature on statistical ecology that this review will overlook [e.g., Steinhorst, 1979; Tiwari, 1979; White and Clark, 1979]. There will also be occasional reference to the study of groundwater quality (recently reviewed by Yeh [1986]), estuarine water quality, and the adjacent disciplines of biotechnology (wastewater treatment) and biomedical systems analysis.

Above all, the review is not about the elegance of methods for the solution of hypothetical problems. Its overriding concern is with the application of methods that will work in the difficult, usually inelegant, but highly enriching area of solving the problems of field case studies.

\subsection{The Issues}

There has always been uncertainty, but it was not really an issue in water quality modeling before the late 1970's. The beginnings of its more systematic and explicit analysis, however, go back further than that. For instance, Bellman et al. [1966] appear to have been among the first to address problems of system identification, or inverse problems, in the field of ecology, other early contributions having been made by Koivo and Phillips [1971], Parker [1972], and Shastry et al. [1973]. O'Neill [1973], as already indicated, and Argentesi and Olivi [1976] were instrumental in bringing the terms error analysis, or uncertainty analysis, to the fore in the context of studying prediction error propagation.

Few would previously have associated the word uncertainty with the problem of system identification, although the way in which a model is derived from, or evaluated by reference to, the in situ field data must clearly influence both its ability to predict future behavior and the confidence to be attached to that prediction. Fewer still would have previously associated system identification (or much more narrowly, model calibration) with the problems of decision making and management. An earlier view of system identification is summarized in the following quotation from DeLucia and McBain [1981] on a case study of managing water quality in the St. John River in the United States.

Recognizing that a model is only an approximation of the real system, it appears logical to visualize that water quality modeling is merely curve fitting in a river system.... [The] statistical theory of estimation and hypothesis testing are all useful in model building.... On the other hand, failure to recognise the quality modeling as curve fitting has, in part, contributed to the 
making of water quality modeling a field of ambiguity and mystery. Too often the calibration/verification procedure is described as a distinct and creative step in water quality modeling. However, it is merely, in fact, an ad hoc procedure to fill partially the role of estimation and hypothesis testing.

Misconceptions can, and do, occur.

1.2.1. Management and science. The concern of environmental management, based, we assume, on environmental science, is the application of knowledge of the relationships between causes and effects in guiding decisions about (1) the restoration of an "acceptable quality" to a damaged aquatic environment, (2) the prevention of damage to an environment as a result of contemplated development, and (3) the operational maintenance of an acceptable environmental quality in the face of seasonal variability and the occurrence of accidents, failures, and extreme events.

Few would disagree that in the light of these objectives there should be as little ambiguity and uncertainty as possible in the relationships between causes and effects. Nevertheless, it is easy to challenge the usefulness of any modeling exercise to decision making, for there is little published evidence with which to support its relevance [Beck, 1985a]. What is worse, the problems of system identification and the analysis of uncertainty are still further removed from the (political) decisionmaking process and therefore still more easily dismissed as irrelevant abstractions.

Much depends on how the "scientist" communicates with the "manager." (These are understood as roles assumed by the individual; they are not mutually exclusive.) It may not be necessary to burden the manager with the details of an analysis of error propagation. But most certainly it is important to be concerned about the scientific basis underpinning the model and its predictions, and this is becoming acutely important given the impressive and persuasive technology of communication now within reach [Loucks et al., 1985; Fedra and Loucks, 1985]. At the same time, it is surprisingly difficult to convince the scientist, let alone the manager, that system identification and the analysis and interpretation of field data are integral to the development of scientific theories about the behavior of complex environmental systems [Young, 1978; $B e c k, 1982,1985 b]$. They are (arguably) the "distinct and creative step" that the above quotation denies. It is essential for system identification to involve a critical questioning of, and creative speculation about, prior hypotheses; to do otherwise is to ignore the role of experiment in theory development. System identification is not "merely curve fitting," if that is the end in itself; it is, if anything, curve fitting as a means to an end, where the end is the rigorous, scientific interpretation of field data. This paper takes the view that there is no obligatory need of system identification in the application of models to the resolution of management issues. But if management calls for a model, it is better that the way in which the model is to be developed and evaluated is agreed generally to be on a sound scientific footing. And without system identification, in its broadest sense, the process of model develpment and evaluation should not be accorded the label of "scientific." In some ways, then, this is little more than what has already been discussed elsewhere on the relationship between management and science [Thomann, 1982].

1.2.2. Classes of models. For certain philosophical and methodological reasons, it is convenient to distinguish between three classes of models, developed as follows. It is obvious that all environmental systems are inherently of a distributed-parameter form. If the analyst were to attempt as complete a conceivable description of the system as possible, we would have the following form of model.

Class I

$$
d \mathbf{x}(t, \mathbf{r}) / d t=\mathbf{f}\left\{\nabla^{2} \mathbf{x}, \nabla \mathbf{x}, \mathbf{x}, \mathbf{u}, \boldsymbol{\theta} ; t, \mathbf{r}\right\}
$$

Here $\mathbf{x}$ is the state vector, i.e., physical, biochemical, and ecological attributes of water quality, $\mathbf{u}$ is a vector of measured input disturbances, $\boldsymbol{\theta}$ a vector of model parameters (coefficients), $t$ is (continuous) time, and $\mathbf{r}$ is a vector representing the three spatial directions (a list of symbols used in the paper is given in the notation list).

From the points of view of the available in situ observations and the applicable methods of system identification, the question is to what extent must simplifying assumptions be made to the description of (1) in order to formulate an identification problem capable of solution. We shall assume that this means in practice either a finite-element or a finite-difference approximation where spatial variability is accounted for by an appropriate redefinition of the state vector, i.e., $\mathbf{x}$ may include elements for the same attribute of water quality in several spatial volumes. Here the finite-element approximation would give the model for the state variable dynamics as

Class II

$$
d \mathbf{x}(t) / d t=\mathbf{f}\{\mathbf{x}, \mathbf{u}, \boldsymbol{\alpha} ; t\}+\xi(t)
$$

with (output) observations of the states given by

$$
\mathbf{y}\left(t_{k}\right)=\mathbf{h}\left\{\mathbf{x}, \boldsymbol{\alpha} ; t_{k}\right\}+\boldsymbol{\eta}\left(t_{k}\right)
$$

where now $\alpha$ is the vector of model parameters relating to this lumped form of model ( $\alpha$ may vary with time $t$ ), $\mathbf{y}$ is the vector of measured output response variables, $\xi$ is a vector of unmeasured, possibly random, input disturbances, and $\boldsymbol{\eta}$ is a vector of random output measurement errors. The argument $t_{k}$ in $(2 b)$ indicates the pragmatic restriction of the observations $\mathbf{y}$ to discrete instants of time (the same is in fact the case for $\mathbf{u}$ in $(2 a)$ ).

The nature of (2), which is central to the remainder of the paper, places the discussion of this review firmly in the conceptual framework of control theory. This is perhaps an unfamiliar framework, but it should not cause undue difficulties in understanding the problems at hand (except possibly in section 7).

A third class of commonly encountered model is the input/ output, transfer function model defined by the discrete-time, difference equation

\section{Class III}

$\mathbf{y}\left(t_{k}\right)=\mathbf{f}\left\{\mathbf{y}\left(t_{k-1}\right), \cdots, \mathbf{y}\left(t_{k-n}\right), \mathbf{u}\left(t_{k-1}\right), \cdots, \mathbf{u}\left(t_{k-n}\right)\right.$,

$$
\left.\boldsymbol{\omega}\left(t_{k-1}\right), \cdots, \boldsymbol{\omega}\left(t_{k-n}\right), \boldsymbol{\beta}\right\}
$$

in which $\boldsymbol{\beta}$ is the relevant model parameter vector and all the sources of error (other than prior parameter estimation errors) are lumped under the definition of the single noise process $\omega$.

1.2.3. A taxonomy of uncertainty. As we have said, the analysis of uncertainty associated with the class II models introduced above will be the central concern of this review.

It is now necessary to relate the sources of error and uncertainty implied by (2) to the basic scheme of Figure 1, i.e., to define a "taxonomy of uncertainty" (a term borrowed from Alcamo and Bartnicki [1985]). At least until near the end of the review (in section 8), uncertainty will be understood in a probabilistic sense, encapsulating therefore the variability in the outcome of a random event (including the attributes of a 


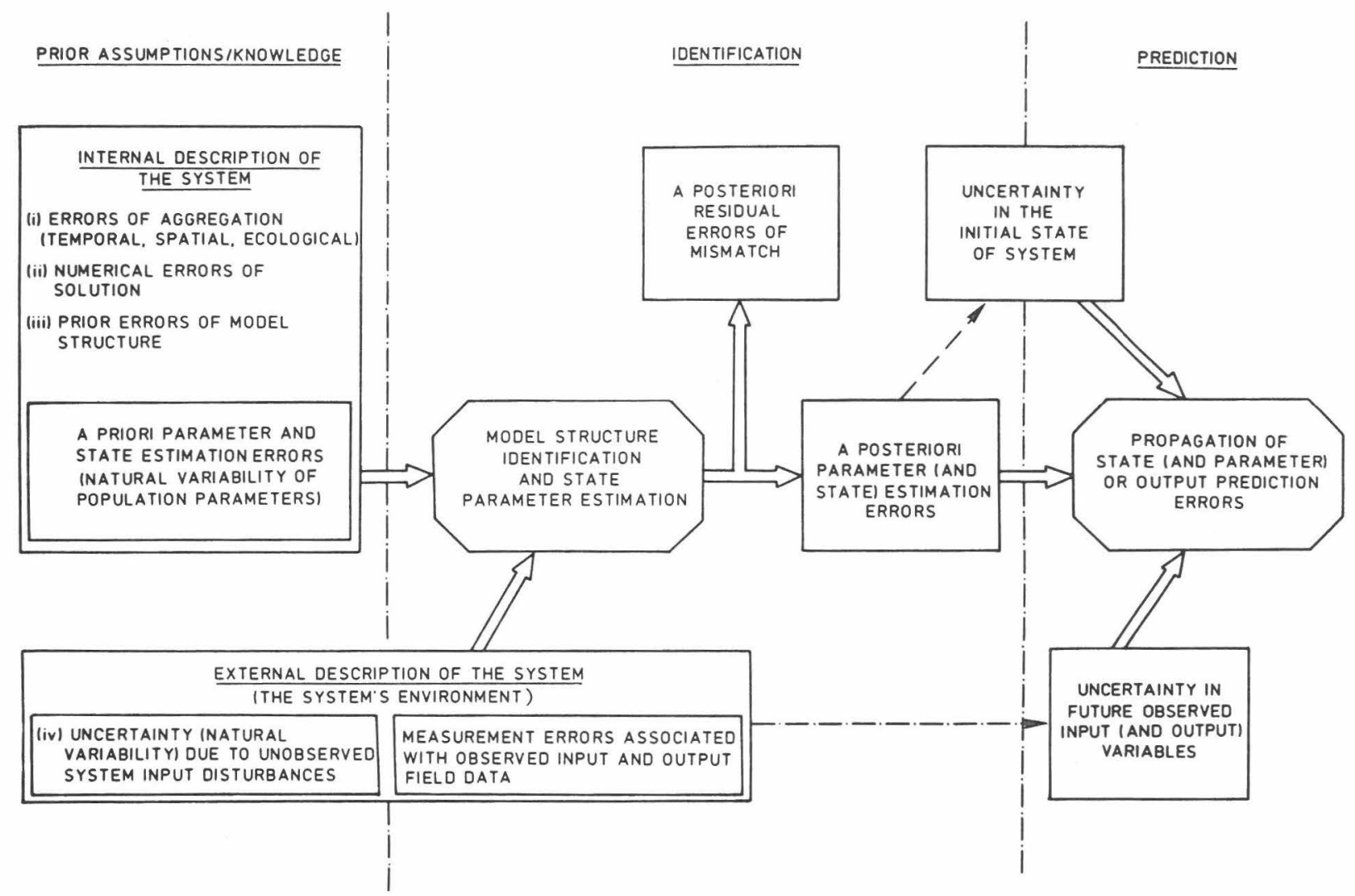

Fig. 1. Frame of reference for the analysis of uncertainty.

biological population) and embracing the notions of erroneous assumptions or the distribution of errors associated with observed or estimated quantities.

There are three perspectives from which to view Figure 1: (1) as providing the logical connections between the cyclical triplet of (prior assumptions $\rightarrow$ identification $\rightarrow$ prediction) and thus the propagation of uncertainty in the unfolding of these procedures, (2) as the taxonomy itself (see below), and (3) as distinguishing between uncertainty associated with an external and an internal description of the system's behavior.

Most of the details of Figure 1 are self-explanatory and will be amplified fully as the review proceeds. There are two exceptions, however, both relating to the uncertainty of the internal description of the system. First, errors of aggregation, especially in the spatial and ecological senses implied by the lumping approximations of a class II model, will only be mentioned in passing. Some work on the errors of aggregation resulting from the approximation of a three-dimensional spatial continuum by a two-dimensional model representation has been reported by McLaughlin [1985] for groundwater systems. Others have investigated in depth the errors arising from the aggregation of groups of heterogeneous biological species into single ecological "compartments" [O'Neill and Rust, 1979] (see also section 7). Second, the errors of model structure, although the focus of much discussion throughout the review, are in fact extremely difficult to quantify in a formal manner. Technically, within the context of the class II model defined above in (2), they could be subsumed under the definition of either the parameter estimation errors or the sources of uncertainty lumped in the definition of $\xi$, ostensibly the "unobserved system disturbances." The latter is conceptually perhaps the more satisfactory means of accounting for such uncertainty, although it has rarely been used for this purpose and is in any case subject to notoriously arbitrary choice (as we shall see in section 4).

To summarize the taxonomy, the sources of uncertainty most usually accounted for are uncertainty in the initial state of the system, uncertainty in the model parameter estimates, uncertainty in the observed input disturbances and output responses, and uncertainty arising from unobserved input disturbances of the system.

1.2.4. Some philosophical points: different models for different roles. Overall, a Popperian view of the scientific method is assumed in this review, although hopefully not in the "naive" sense discussed by Chalmers [1982]. The relevant consequences of this are several.

First, Popper [1968] has drawn a distinction between science and nonscience as a matter of whether the hypotheses associated with any attempt at a description of nature can be formulated in a manner whereby they can be unambiguously falsified. Given the currently available field observations of the behavior of environmental systems, "comprehensive" models (of the class I type), which have become enormously complex assemblies of very many hypotheses, cannot be effectively falsified. This is partly a function of uncertainty in the field data, certainly a function of current limitations in the methods of system identification, and essentially a function, in the event of demonstrating a significant mismatch between the model and observations, of being unable to distinguish which among the multitude of hypotheses have been falsified. In fact the detailed spatial patterns of water circulation and equally detailed differentiation of ecological behavior described by the more complex models would demand experimental observations that are simply not technically feasible. This is clearly a problem intrinsic to the unavoidable process of aggregation, especially between class I and class II models, and specifically 
deriving from the fact that the dimensions of the input/output observations $\{\mathbf{u}, \mathbf{y}\}$ are (much) smaller than those of the state and parameter vectors $\{\mathbf{x}, \boldsymbol{\alpha}\}$.

Second, this facilitates a more "comfortable" view of models as either formalized archives of hypotheses or as vehicles for the exhaustive analysis and interpretation of data. The debate about preferred approaches to modeling can therefore be shifted away from the customary confrontations between the large and the small or the statistical and the mechanistic models. Such a view reconciles the role of the class I model, as the archive, with the role of the class III model, often the only vehicle for the analysis of data. It allows one to acknowledge that in seeking an understanding of the system's observed behavior, a class III model is not a satisfactory end point to the analysis. It is instinctive for the scientist to ask how and why certain types of behavior or anomalies are observed, not merely to accept that they are. For instance, a linear (regression) relationship identified between the concentrations of aluminium and hydrogen ions in an upland stream will prompt many questions, since it runs counter to the expected deductions from chemical equilibrium theory [Whitehead et al., 1986]. The interpretation of anomalies and the revision of inadequate hypotheses are not therefore matters that can be resolved without recourse to the archive of hypotheses associated with a class I model. In fact, there is an important interplay between the two sides of the dichotomy.

Third, in light of the above, it is convenient to adopt as an organizing principle for the procedure of system identification the following complementary (and iterative) questions of how to expose the failure (inadequacy) of the constituent hypotheses of a model structure, and how to infer the form of an improved model structure from diagnosis of the failure of an inadequate structure and from the prior knowledge associated largely with the class I models.

In answering these questions, the class II models play a central role as intermediaries between the other two classes of models. In spite of their aggregated form, they still embody the spirit of the hypotheses about those phenomena thought to govern system behavior and in a form for which the identification problem is capable of solution.

1.2.5. Hydrology and water quality. It is also instructive to draw a distinction between hydrological system identification and environmental (water quality) system identification. The essential problem of hydrological management in the narrow sense of having the right quantity of water in the right place at the right time is one that has always been present and, as a problem for study, unchanging. Cause and effect in hydrology are unambiguously related (precipitation causes runoff and hence streamflow), although undoubtedly the precise mathematical form of this relationship can be extremely difficult to identify for complex hydrological systems. Cause and effect are not always self-evident in managing water quality; and the essential problems of water quality have changed and continue to change significantly (first it was easily degradable organic wastes, then eutrophication, then the nitrate problem, and now toxics and acid rain [Beck, 1985a]). The emergence of new problems causes shifts of analytical study from one area to another and lessens therefore the effort devoted to analyzing the "classical" problems in greater detail, including greater statistical detail. Consider, for instance, a model relating rainfall-runoff to observed precipitation and a residual noise process. Hydrology has advanced to the point where one would be much concerned about the assumptions made about the stochastic processes affecting the identification of this model. The analyst of water quality problems would be content to make any convenient assumption about such processes providing there was available a robust estimator for establishing in the first place the relationship between the relevant input and output system characteristics. Moreover, it may well be that water quality will always be so concerned because it is in the nature of the subject that analysis is more usually directed at the determination of new relationships among new sets of variables rather than continual refinement of models for the same relationships among the same pairs of variables.

In effect, almost all the problems of environmental system identification can be viewed as problems of model structure identification, and although they are here not treated under that title, many of the methods and case studies discussed in sections 5 and 6 are relevant to that problem.

1.2.6. Uncertainty, ambiguity, and identifiability. Until the issue of uncertainty in water quality modeling had risen to significance in the late 1970 's, it had been the paradigm to develop as comprehensive a "physics-based, mechanistic" representation of the system as possible [e.g., Park et al., 1974; Chen and Smith, 1979]. Our concern about such models, and effective tests of their many associated hypotheses, have already been noted both above and elsewhere [Reckhow and Chapra 1983a]. That the constituent hypotheses of these models cannot be effectively falsified can be stated alternatively as a lack of identifiability, as a case of overparameterization, or that the model contains surplus content [Young, 1978]. The crux of the problem is that what one would like to know about the internal description of the system $\{\mathbf{x}, \boldsymbol{\alpha}\}$ is of a substantially higher order than what can be observed about the external description of the system $\{\mathbf{u}$, $\mathbf{y}$. The model may contain descriptions either of a type of behavior not actually observed in the particular sample of data, or of multiple types of behavior, the individual components of which cannot be disentangled from observations of their collective effect. The consequences are usually apparent in the absence of a uniquely "best" combination of parameter values that fit the data (many combinations are "equally good") and in parameter estimates with high error variances and covariances.

On philosophical grounds the problem of model identifiability is clearly undesirable. It implies an uncertain and ambiguous interpretation of past observed behavior and, equally so, the possibility of ambiguous (and even contradictory) predictions from a given model. Identifiability will subsequently emerge as the dominant problem of model parameter estimation in section 5 (see also Sorooshian et al. [1983], Sorooshian and Gupta [1983], and Gupta and Sorooshian [1983] for an exhaustive treatment of the problem in hydrological models). It is also the key conceptual link between the topics of identification and prediction that form the two halves of this review.

But this now is as far as it is necessary to go in discussing the issues for review. The casual reader, if still with us, we shall rejoin in section 8 .

\section{IdENTIFIABILITY AND EXPERIMENTAL Design}

In less philosophical and more quantitative terms, what can be done to detect and avoid the potential problems of identifiability?

The problem lies in the "choices" of $\{\mathbf{x}, \boldsymbol{\alpha}\}$ and $\{\mathbf{u}, \mathbf{y}\}$ or, in 
other words, in inappropriate choices of model structure (prior theory) and the set of input/output variables to be (or that have been) observed in a planned experiment. Some of the difficulties arise solely from the form of the model structure, some are obviously difficulties with the awkward properties of the data, and others arise from particular combinations of the two. In any case, the problems of identifiability are intimately related to the issue of experimental design, or problem (P4) as defined in the introduction: the design of experiments, or monitoring programs, for the specific purpose of reducing critical uncertainties associated with a model.

In fact, in answer to our question, very little can be done a priori to detect and avoid subsequent identifiability problems, and for three very practical reasons.

1. Planned experiments are in general not possible for environmental systems; there is usually little freedom to choose $\mathbf{u}$ and $\mathbf{y}$, to isolate a single cause-and-effect couple for experimentation along the lines of laboratory science, or to design the experimental perturbations in $\mathbf{u}$ (as would normally be assumed in the relevant literature of control theory [e.g., Gustavsson, 1975; Goodwin and Payne, 1977; Isermann, 1980]). There are exceptions, however, notably dye-tracer studies, which will be discussed below [e.g., Jakeman and Young, 1980; Beer and Young, 1983], the use of tubular enclosures in lakes [Lack and Lund, 1974], and the whole-lake experiments in eutrophication reported by Schindler and co-workers [Schindler and Fee, 1974; Schindler et al., 1978].

2. A good experimental design requires good prior knowledge of the system's behavior, i.e., a good model, which begs the original question.

3. Formal analysis of the identifiability of a model structure [Bellman and Aström, 1970] does not appear to have yielded any easily computable procedures (as noted by Cobelli et al. [1979]); it leads to a cumbersome computational effort for all but the simplest problems [Holmberg, 1981; Gentil, 1982] and is often approached within the (unfamiliar) context of frequency-domain representations of system behavior [ Godfrey et al., 1982].

Most first attempts at the design of sampling programs will not be addressed to the problem of system identification as discussed here. They will usually derive from the exclusive interests of management [Reckhow, 1978; Ellis and Lacey, 1980; Ward and Loftis, 1983] and will not involve any more complicated prior knowledge than some elementary statistical models for the distribution of random variables.

The one area of water quality modeling in which considerable prior knowledge is available and where deliberate experimentation is possible is that of the identification of pollutant transport and dispersion. Experimental design in this area has accordingly attracted quite detailed analysis, specifically from the point of view of system identification, and indeed exploits the fact that uncertain and poorly estimated parameter values result from a model structure that is overparameterized [Jakeman and Young, 1980; Beer and Young, 1983]. The analysis is, however, restricted to particular forms of model structure, in fact the single input/single output versions of the class III models of (3). But what it shows is of considerable practical relevance, for it suggests that an "optimal" injection point for the tracer would be located some distance upstream of the system (the stretch of river) under study. The intermediate length of river prior to the system input then has the physical effect of filtering out a portion of the noise processes that would otherwise degrade the capacity to identify a model from

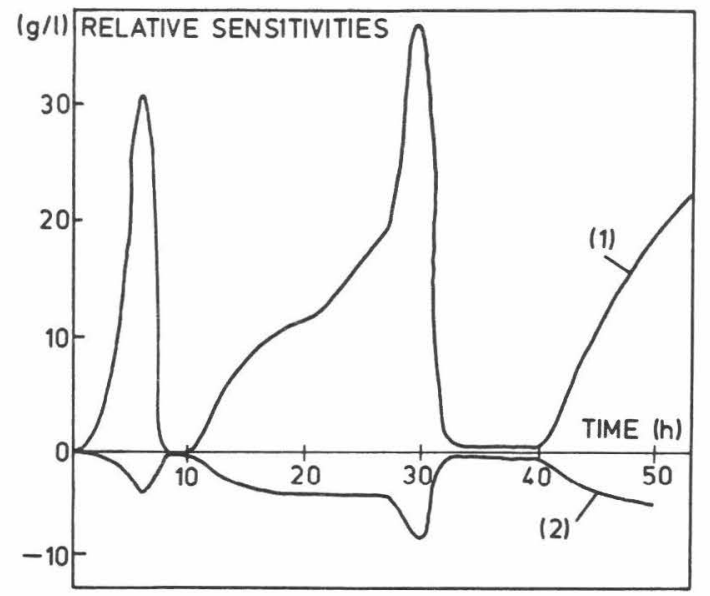

Fig. 2. Relative sensitivities for the change in estimated biomass concentration (in $\mathrm{g} \mathrm{L}^{-1}$ ) that would result from changes in the maximum specific growth rate constant (curve 1) and the saturation concentration (curve 2) [after Holmberg, 1981].

the field observations [Jakeman and Young, 1980]. Closely similar conclusions have also been reached from quite a different perspective by van Straten et al. [1985].

It is not surprising that the conditions governing identifiability are closely related to sensitivity analysis, and while not wishing to pre-empt the discussion of section 7, there are certain general observations that are best made here. They refer to the identifiability of the Monod kinetic expression for biological growth, which although quite specific, is of sufficiently general importance because of its widespread use in ecological, fermentation, and biomedical system models. Figure 2 shows the relative sensitivities for the change in estimated biomass concentration (in grams per liter) that would result from changes in the maximum specific growth rate constant (curve 1) and the saturation concentration (curve 2) in a model of a continuous culture fermentation process [Holmberg, 1981]. The qualitatively identical fluctuations in these two relative sensitivity coefficients would cause the associated parameters not to be uniquely identifiable; errors in the values of the two parameters would tend to be mutually self-cancelling in terms of the net model response and therefore not detectable. Note that such an analysis deals only with the intrinsic properties of the model, i.e., the internal description of the system's behavior $\{\mathbf{x}, \boldsymbol{\alpha}\}$, and makes no reference to any particular set of field data, other than that biomass concentration would need to be an observed variable. In fact, given a set of observations from an entirely deterministic simulated system (the noise processes $\xi$ and $\eta$ being identically zero in (2)), Holmberg and Ranta [1982] have shown further that a typical least squares parameter estimation algorithm has great difficulty in converging to an optimal and unique pair of estimates for the maximum specific growth rate and saturation concentration constants. The essential problem is that the surface of the (squared-error) objective function has the shape of a long, narrow, steep-sided valley running roughly parallel to the axis of the saturation concentration constant in the two-dimensional parameter space. In other words, in the neighborhood of its minimum, the value of the objective function is virtually insensitive to the value of the saturation concentration, and hence many pairs of values for the two parameters constitute effectively the least squares estimates.

There have been other studies on the use of sensitivity coefficients to establish the identifiability of a model, for instance, 
Gentil [1982] in a case study of Lake Aiguebelette in France (her paper is also of interest for its discussion of identifiability, observability, and controllability as concepts in linear system theory), and to determine an experimental sampling strategy for model identification [Vialas et al., 1985].

However, without considerable prior knowledge of model structure and model uncertainty, there is little of substance that can be said of identifiability and experimental design for system identification, and we shall defer further discussion of identifiability, in particular, until section 5. In the hydrological sciences more generally, the subject of identifiability has received barely any attention until relatively recently [e.g., Sorooshian and Gupta, 1985]. Should it become more relevant, there is much to be learned from the record of its parallel study in the adjacent disciplines of biotechnology and biomedical systems analysis [Godfrey and Distefano, 1985].

\section{Generating Preliminary Hypotheses}

We come then to the problem of model building in its pristine state, with little confident, prior knowledge and few experimental observations. Broadly, this is the first of our four problem areas as defined in the introduction (section 1), i.e., problem (P1): uncertainty about the relationships among the variables characterizing the dynamic behavior of systems, i.e., uncertainty about model structure.

The issue is one of how to get started, of how to start, in particular, against a background of gross uncertainties.

We noted earlier the preferred view of system identification as separating into the dual procedural steps of (1) exposing the failure of inadequate, constituent model hypotheses, and (2) speculating about, generating, or inferring the form of improved hypotheses.

The exposure of failure, which is a relatively "crisp" concept, implies both bold, confident prior hypotheses and the availability of adequate time-series observations of the inputs (u) and outputs $(\mathbf{y})$. But this is rarely the case. It is much more common that a few quantitative observations are available (probably sampled irregularly and infrequently) together with less quantitative, more qualitative, empirical evidence of the system's behavior. In such situations (the subject of this section) it is apparent that the problem is one of generating some preliminary hypotheses about the possible mechanisms governing qualitatively observed behavior. The approach to such problems, as described below, is a speculative exercise. And although it is convenient to think of it as an implementation of the second of the above two procedural steps of system identification, in practice the approach operates on a principle of sifting through a set of prior hypotheses and rejecting from further consideration those to which observed behavior appears to be insensitive.

The approach is due collectively to Hornberger, Spear, and Young [Young et al., 1978; Hornberger and Spear, 1980, 1981; Spear and Hornberger, 1980; Young, 1983] and has variously been labeled a regionalized sensitivity analysis, a procedure for hypotheses generation, and speculative simulation modeling, names that themselves are revealing of the interwoven concepts of this review. The approach is stated most generally and succinctly by Hornberger and Spear [1981]. It is placed properly in the context of modeling "poorly defined" systems by Young [1983], much the same as here, and it is best illustrated by a case study of cultural eutrophication in Peel Inlet, western Australia [Hornberger and Spear, 1980; Spear and Hornberger, 1980].
For want of a better name, we shall refer to it here under the rubric of the Hornberger-Spear-Young (or HSY) algorithm. It is not really an algorithm, and we shall leave poster. ity to invent a more adequate title.

\subsection{The Hornberger-Spear-Young Algorithm}

Let us begin, perhaps paradoxically, by giving a definition of the familiar problem of parameter (and state) estimation, typically for a class II model.

Given a set of experimental, time-series field data comprising the measured inputs, $\mathbf{u}\left(t_{k}\right)$, and the measured outputs, $\mathbf{y}\left(t_{k}\right)$, of the system, determine values for the model parameters, $\alpha(t)$, and state variables, $\mathbf{x}(t)$, such that some (error, loss, or objective) function of the differences between the estimated $(\langle y\rangle)$ and observed $(\mathbf{y})$ output responses is minimized.

For the present this is a highly restrictive problem definition. It does not capture at all the situation in which the field data are sparse, but it can be made to do so by making the following two fundamentally important substitutions, as proposed by Hornberger, Spear, and Young.

1. The trajectories of the time-series observations $\left\{\left(\mathbf{y}\left(t_{0}\right)\right.\right.$, $\left.\mathbf{y}\left(t_{1}\right), \cdots, \mathbf{y}\left(t_{N}\right)\right\}$, against which the performance or the model is to be evaluated, are replaced by a definition of (past) behavior $(B)$ in terms of less detailed (more qualitative) constraints derived from the limited available observations (thresholds, topological constraints, and logical constraints, among others, are permissible).

2. The error-loss (objective) function for locating a unique and best estimate $\langle\boldsymbol{\alpha}\rangle$ of the parameter vector is replaced by a criterion that either accepts or rejects a sample vector $\alpha^{*}$ as giving rise to the past behavior $(B)$ defined according to point 1 , above.

In other words, the model is required, as it were, to pass through a "corridor" of constraints with "hurdles" to be overcome (as in the most simple form of Figure $3 c$ ), and it either succeeds or fails. (a)

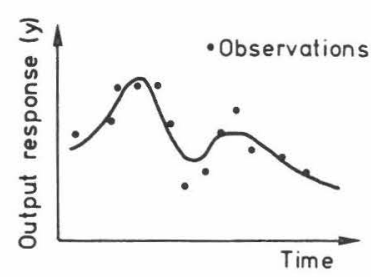

(c)

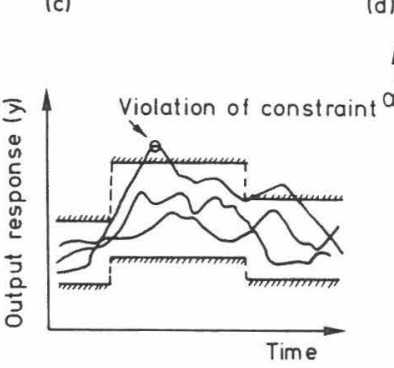

(b)

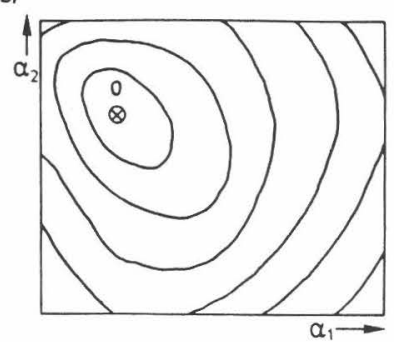

d) Random realizations of parameter

Fig. 3. A comparison of $(a$ and $b)$ the concepts of estimation and ( $c$ and $d$ ) the HSY algorithm: $(a)$ fitting the model response to the data, $(b)$ contours of the fitting-function surface in the parameter space, $(c)$ specification of constraints on acceptable model responses, and $(d)$ analysis of model parameter values (dots indicate values giving rise to acceptable behavior, and crosses indicate values giving rise to unacceptable behavior). 
For example, to quote from the original study of Peel Inlet, one item of the behavior definition $(B)$ was chosen to constrain the estimated yearly peak biomass of the nuisance alga Cladophora to be greater than 1.5 times and less than 10.0 times its initial biomass at April 1 (defined as time $t_{0}$ ), i.e.,

$$
1.5 x\left(t_{0}\right) \leq x_{\max }(t) \leq 10.0 x\left(t_{0}\right)
$$

In addition, the ranges of permissible values from which the sample model parameter vectors are to be drawn were specified as rectangular distributions with upper and lower bounds, i.e.,

$$
\alpha_{l} \leq \alpha^{*} \leq \alpha_{u}
$$

The two types of inequalities (4) and (5) reflect the uncertainty of the empirical evidence and the uncertainty of the prior hypotheses, respectively.

The procedure of the analysis is a form of Monte Carlo simulation. In the original study the pattern of input disturbances $\mathbf{u}(t)$ and the initial conditions $\mathbf{x}\left(t_{0}\right)$ were assumed to be known and not subject to uncertainty (assumptions that are not restrictive, as we shall see later). And since $\boldsymbol{\xi}(t)=\mathbf{0}$ was also assumed for $(2 a)$ of a class II model, it is apparent that all the uncertainty of the problem can be lumped under the title of parameter uncertainty. A sample vector $\alpha^{*}$ is drawn at random from its parent distribution and substituted in the model of (2) to obtain a sample realization of the trajectory $\mathbf{x}(t)$, which is then assessed for its satisfaction, or otherwise, of the set of constraints defined in the form of inequality (4). Repeated sampling of $\alpha^{*}$, for a sufficiently large number of times, allows the derivation of an ensemble of parameter vectors that gives rise to the behavior $(B)$ and a complementary ensemble associated with not-the-behavior $(\bar{B})$. For this analysis therefore there is no meaningful interpretation of a degree of closeness to a uniquely best set of parameter estimates. Each sample vector $\alpha^{*}$ giving rise to the behavior is equally as "good" or as "probable" as any other. The crux of the analysis, with regard to resolving the questions of our problem (P1), in the introduction, is the identification of which among the hypotheses parameterized by $\alpha$ are those that are significant determinants of observed past behavior. "Significance" is here indicated by the degree to which the central tendencies of the marginal and joint distributions of the (a posteriori) ensembles of the "behavior-giving" parameter values $\alpha^{*}(B)$ and their complement $\alpha^{*}(\bar{B})$ are distinctly separated. Thus, for instance, the distinct clustering of parameter combinations that give the behavior, toward high values of $\alpha_{2}$ and low values of $\alpha_{1}$ in Figure $3 d$, suggests that the hypotheses associated with $\alpha_{1}$ and $\alpha_{2}$ are likely to be fruitful speculations in understanding the observed system behavior. Rank ordering of the separation of the distributions of $\alpha_{i}^{*}(B)$ and $\alpha_{i}^{*}(\bar{B})$ for each individual parameter $i$ allows the rejection of some of the hypotheses as probably insignificant [Hornberger and Spear, 1981]; they might alternatively be said to be part of the surplus content of the model, a point discussed earlier in the introduction (section 1) with respect to the problem of identifiability.

The speculative character of the analysis should be obvious. The objective is to generate a preliminary set of promising hypotheses about a system's behavior. The origins of the term regional sensitivity analysis, as opposed to a local sensitivity analysis (as in the work by Jorgensen et al. [1978], Rinaldi and Soncini-Sessa [1978], and van Straten and de Boer [1979]) should also be apparent. A local sensitivity analysis is usually concerned with determining the changes in the state variable trajectories (in the neighborhood of a set of nominal reference trajectories) that would result from small changes in the values of the parameters. The regional aspect of the approach outlined above is its evaluation of the sensitivity of a broad range of possible realizations of the state trajectories to (nonlocal) ranges of values for the parameters. For example, had there been no clustering discernible in Figure $3 d$, an intuitive conclusion would have been to say that the behavior definition is not sensitive to any particular values of $\alpha_{1}$ and $\alpha_{2}$. Finally, we may note that the focus on hypotheses parameterized by $\alpha$, as opposed to the tendencies of the specific values assumed by $\alpha^{*}(B)$ and $\alpha^{*}(\bar{B})$, concentrates the analysis on problems of type (P1) and not on those, i.e., parameter estimation, of type (P2).

\subsection{Case Studies}

The significance of the work conducted by Hornberger, Spear, and Young is readily apparent from the number of other studies that have already adopted a similar approach. Van Straten [1981] has applied it in order to evaluate a hypothesis of phosphate sorption on particulate matter and the association of this mechanism with the exchange of nutrients between sediments and water in a shallow lake (Lake Balaton, Hungary). Halfon and Maguire [1983] report results for a study of the fate of fenitrothion (a pesticide used to control spruce budworm) in an aquatic environment, and Whitehead and Hornberger [1984] have used the approach to examine certain aspects of algal population dynamics in the Thames River basin in United Kingdom (see also section 4).

All of these case studies do not deviate significantly from the basic approach outlined above. Fedra, however, has been prominent in extending the approach in other important directions.

1. In formally adjoining bounded distributions for $\mathbf{u}(t)$ and $\mathbf{x}\left(t_{0}\right)$ in (2) to the parameter distributions of inequality (5), i.e., hypotheses about the patterns of $\mathbf{u}(t)$ and $\mathbf{x}\left(t_{0}\right)$ and their uncertainty are parameterized via $\alpha$ [Fedra et al., 1981; Hornberger and Spear, 1981; Hornberger and Cosby, 1985a].

2. In deliberately associating the residual (a posteriori) uncertainty of an "identified" model with subsequent analysis of prediction uncertainty [Fedra et al., 1981; Halfon and Maguire, 1983; Hornberger and Cosby, 1985a] (see also section 7).

3. In using the approach to address much more explicitly the problem of model structure identification, as defined in section 4 [Fedra, 1981].

4. In combining the use of error (objective) functions with the behavior definition as a means of broadening the concept of determining acceptable model performance [Fedra, 1983; Hornberger and Cosby, 1985a; Hornberger et al., 1985].

Fedra's style is typically philosophical and provocative, and the reader will not be disappointed by the synthesis he has given his ideas in Fedra [1983], where he uses as case studies a problem of lake eutrophication in the Attersee, Austria [Fedra et al., 1981], a pelagic food web in the North Sea [Fedra, 1981], and a simple rainfall-runoff model for a small mountainous watershed in upper Austria.

\subsection{Commentary}

The appeal and power of the HSY algorithm are undeniable. Simplicity and flexibility of method, together with the enforced declaration of arbitrary assumptions (as Fedra has observed) are virtues rarely matched by most of the other topics of this review. Above all, the approach occupies a previously empty niche in the analysis of uncertainty, identification, and prediction. Its applicability is essentially independent of the complexity of the model structure, since the classi- 
fication scheme remains a simple binary system of giving, or not giving, rise to the behavior defined.

There are, however, some disadvantages. First, the interpretation of the derived a posteriori parameter distributions becomes more difficult as the dimension of the parameter vector increases, and for all practical purposes, it seems probable that any conclusions will have to be restricted to the properties of, at most, the univariate and bivariate marginal distributions associated with the multivariate joint distribution.

Second, if the primary advantage of an approach is its simplicity, then much of that advantage will be surrendered should it lose the attribute of being simple. Fedra's introduction of what are, in effect, sampled time series of permissible ranges for the state variable trajectories (as opposed to point observations from an associated probability distribution) and his use of terms such as best estimates and estimation schemes that "optimize" are redolent of old habits. They are suggestive of ways in which to use the approach that go against its two cardinal points. There are indeed signs elsewhere [e.g., Hornberger and Cosby, 1985b; Hornberger et al., 1985] of a loss of focus on the original motivations for the development of the approach, i.e., the acute need to handle situations of sparse data and to generate preliminary hypotheses about a system's behavior. These more recent developments have dealt with extensive (notably hydrological) data sets and the problems of identifiability and surplus content, and it is again the encroaching complication of what is supremely a simple approach that gives cause for concern.

Third, Sharefkin's [1983] criticism that the approach lacks refinement in its crude binary classification procedure is also a tempting, but possibly counterproductive, step in the direction of complication.

And fourth, when a technique is easy to apply, such facility may mask the rigor that is necessary in other less technical aspects of the analysis. The subtlety and difficulty of the HSY algorithm, and perhaps too the probability of a successful outcome, lie in careful assembly and composition of the hypotheses that go to form the model structure. It is revealing, for example, to contrast the relative richness of the clear-cut conclusions of the Peel Inlet study using a problem-specific model [Spear and Hornberger, 1980], with the relative inconclusiveness of the Attersee project [Fedra et al., 1981], which made use of a model developed for more general, nonspecific purposes (a corruption of the popular computing aphorism would have this: blandness in, blandness out).

\section{Selection and Evaluation of Model Structure}

A more likely conclusion from the foregoing speculative form of analysis is that further more specific, more intensive experimental study should be made of the system. Were this to be the case, thus yielding a set of time-series field data, the selection and evaluation of model structure could then proceed in a more refined fashion. The problem area of interest is still that of problem (P1), though now in the sense of what we shall define as the problem of model structure identification:

The unambiguous determination, by reference to the in situ field data $\left\{\mathbf{u}\left(t_{k}\right), \mathbf{y}\left(t_{k}\right)\right\}$, of how the measured input disturbances $\mathbf{u}$ are related to the state variables $\mathbf{x}$ and how these latter are in turn related both among themselves and to the measured output responses $\mathbf{y}$ of the system under study.

This is still quite a broad problem definition and certainly much broader than the problem of estimation as defined pre- viously in section 3. It amounts to identification of the functional relationships $\mathbf{f}\{\cdot\}$ and $\mathbf{h}\{\cdot\}$ in (2), and this in turn implies distinguishing among choices for the state $(\mathbf{x})$ and parameter $(\boldsymbol{\alpha})$ vectors.

In relation to the discussion of section 3 , the focus of the problem has changed and, if anything, narrowed somewhat: there are more field observations, fewer gross uncertainties about the observed nature of the system's behavior, and in principle, some more confident prior hypotheses about the mechanisms believed to govern that behavior. The selection and evaluation of model structure are not equivalent to a test of the hypothesis that the model as a whole should be accepted (or rejected). Rather, what is required is a test of the adequacy of each constituent model hypothesis. Yet this test cannot merely be conducted on a part of the model isolated from the whole, any more than the experimental conditions can be so reduced.

So we need a method of solution that can accommodate both the inherently multivariable character of the model and field observations as a whole, yet establish the "success" or failure of any of the individual, constituent hypotheses. This is no easy demand to satisfy. In this section we shall develop a conceptual picture of the problem of model structure identification and then describe how the idea of recursive stateparameter estimation can be used to solve the problem. This presupposes a familiarity with recursive estimation algorithms, which therefore will be introduced shortly. The details of these algorithms are not crucial to an appreciation either of the problem solution or the illustrative case study results, and they are therefore confined to a largely self-contained section (section 4.3), which may be omitted without loss of continuity. This detail, however, is well worth absorbing on at least three accounts. First, it reveals how the propagation of uncertainty influences the process of identification; second, it prepares much of the theoretical ground necessary for the subsequent discussion of prediction error propagation in section 7; and third, it explains the conceptual basis on which the history and origins of this review are founded [Beck and Young, 1976; Young, 1978; Beck, 1982]. But there should be no suspicion in the reader's mind that recursive estimation is a panacea. It is not; it is one among several perspectives on the problem of model structure identification. Nor will the application of such algorithms deliver "automatically" a solution to this problem; any solution requires experienced judgment on the part of the analyst.

\subsection{Definitions of Related Problems}

But, to begin with, model structure identification is not an easily understood term, or rather it is often misunderstood as something other than what is intended here. It is not the same as model-order estimation, which, given the input/output form of a class III model (equation (3)) would normally be defined as

The determination of an appropriate integer value for $n$ (in (3)), i.e., the order of the relevant polynomials in the backward shift (or lag) operator.

Even for this more narrowly and more easily defined problem for which there are relatively systematic procedures of solution [e.g., Box and Jenkins, 1970; Söderström, 1977; Halfon et al., 1979; Hipel, 1981; Young et al., 1980], there is not necessarily any truly "objective" indicator of having identified the best model order, nor are these procedures especially 

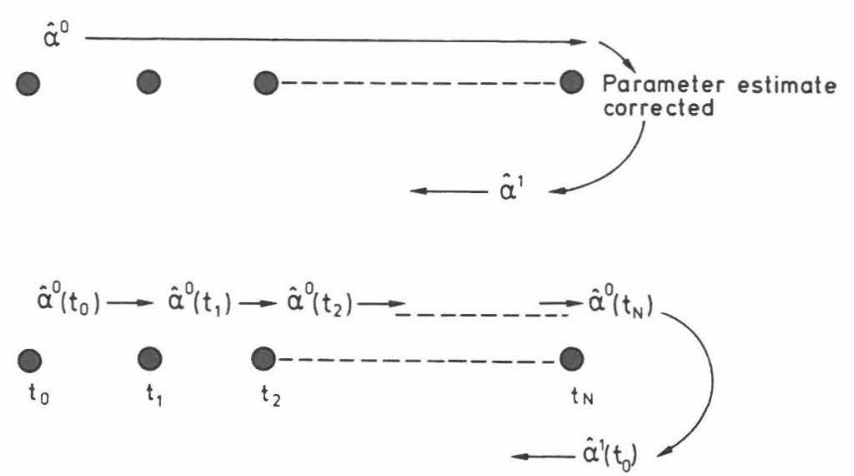

Fig. 4. Methods of parameter estimation (a) off-line and (b) recursive. Superscript $i$ in $\left\langle\alpha^{i}\right\rangle$ denotes the estimate for the $(i+1)$ th iteration through the data.

effective on the data typical of environmental systems [e.g., Beck, 1979a]. Since model-order estimation is usually associated with a class III model structure, it will not be a primary concern of this review.

Model structure identification is not quite the same as model discrimination, defined as

An analysis with the objective of discriminating among competing hypotheses about a system's behavior, i.e., among models with different structures.

Such a definition does not embody the notion of inferring the form of an improved model structure from diagnosis of the failure of an inadequate prior structure, and this as we shall see, is central to our interpretation of model structure identification.

\subsection{Recursive and Batch Estimation Algorithms}

Although the primary concern in this section is with uncertainty about the model structure, access to a resolution of this form of uncertainty is (once again) gained via the estimation of the model parameters (and states).

Consider therefore Figure 4. It illustrates the essential differences between the recursive (on-line) and the more usual batch (off-line, en bloc) forms of data processing algorithms. With a batch procedure (as in Figure 4a), the parameter estimates are assumed to be constant and equal to their a priori values, $\left\langle\boldsymbol{\alpha}^{0}\right\rangle$, while the complete block of time-series field data, from time $t_{0} \rightarrow t_{N}$ of the experimental period, is processed by the algorithm. A loss function, typically based on the errors between the observed and model responses, is calculated at the end of each iteration; the algorithm searches then for suitable directions toward the minimum of the loss function over the parameter space and computes an updated set of parameter values $\left\langle\boldsymbol{\alpha}^{1}\right\rangle$ for substitution into the next iteration through the data (from $t_{0} \rightarrow t_{N}$ ). We shall have much more to say about batch estimation algorithms in section 5 .

A recursive algorithm, in contrast, computes revised parameter estimates, $\left\langle\boldsymbol{\alpha}^{0}\left(t_{k}\right)\right\rangle$, at each sampling instant $t_{k}$ of the field data (Figure $4 b$ ); the minimization of the error loss function is implicitly, rather than explicitly, accounted for in the algorithrns. At the end of the block of data the estimates $\left\langle\alpha^{0}\left(t_{N}\right)\right\rangle$ are substituted for the a priori parameter values $\left\langle\alpha^{1}\left(t_{0}\right)\right\rangle$ of the next iteration through the data. Subsequent iterations through the set of field data may be required, depending upon the nature of the recursive algorithm chosen, or for reasons of short-length data records.

The essential problem of model structure identification is that, given observations of the external description of the system $\left\{\mathbf{u}\left(t_{k}\right), \mathbf{y}\left(t_{k}\right)\right\}$, inference about the internal description of the system is required, i.e., information about the states and parameters $\{\mathbf{x}, \boldsymbol{\alpha}\}$ and the functional relationships $\mathbf{f}\{\cdot\}$ and $\mathbf{h}\{\cdot\}$ in the class II model of (2). Clearly $\mathbf{f}$ and $\mathbf{h}$ cannot be directly identified in some automatic fashion. It is possible only to postulate forms for $\mathbf{f}$ and $\mathbf{h}$, then to estimate the corresponding $\{\mathbf{x}, \boldsymbol{\alpha}\}$, and thence to infer from these estimates the adequacy or otherwise of the choices for $\mathbf{f}$ and $\mathbf{h}$. How precisely this latter might be achieved will be discussed later, but it depends crucially upon the ability to estimate possible variations with time of the model parameter $(\boldsymbol{\alpha})$ estimates; hence the special significance of a recursive estimation algorithm.

\subsection{Uncertainty and State-Parameter Estimation}

All problems of system identification are concerned with this translation of information about the external description of the system into information about the internal description of the system. What has not yet been explored is the question of how the (assumed) uncertainties of these two forms of description influence the process of "translation," i.e., of identification or state-parameter estimation. In order to discuss this point we must now introduce a model for the propagation of errors associated with the state-parameter estimates. This error model is quite general and, together with other models of error propagation, has been discussed extensively by Schweppe [1973]. For our purposes it has particular importance in providing an explicit, quantitative connection between the subjects of identification and prediction.

4.3.1. A model of error propagation. Since the temporal variability of the model parameters is also to play an important role, it is necessary to make some assumptions about how to model this variability. Let us suppose therefore that the state vector dynamics of $(2 a)$ can be augmented by an equivalent expression for the parameter dynamics, so that

$$
\begin{aligned}
{\left[\frac{d \mathbf{x}(t) / d t}{d \boldsymbol{\alpha}(t) / d t}\right] } & =\left[\frac{\mathbf{f}\{\mathbf{x}, \mathbf{u}, \boldsymbol{\alpha} ; t\}}{\mathbf{0}}\right]+\left[\frac{\xi(t)}{\zeta(t)}\right] \\
\mathbf{y}\left(t_{k}\right) & =\mathbf{h}\left\{\mathbf{x}, \boldsymbol{\alpha} ; t_{k}\right\}+\boldsymbol{\eta}\left(t_{k}\right)
\end{aligned}
$$

in which the augmented state-parameter vector $\mathbf{x}_{a}$ would be defined as

$$
\mathbf{x}_{a}{ }^{T}=[\mathbf{x}, \boldsymbol{\alpha}]
$$

where superscript $T$ denotes the transpose of a vector or matrix. The assumed model for the variations of the parameters with time is that they vary in an unknown, random-walk fashion (more specific models are, of course, possible). The random process $\zeta$ in $(6 a)$, representing the sequence of unknown "parameter disturbances," is an additional source of uncertainty; it reflects the intensity of the variability to which any parameter is expected to be subject. Clearly if the parameters of the model are thought a priori to be truly constant, then $\zeta(t)=\mathbf{0}$ for all $t$ should be assumed, although this does not imply that the recursive estimates of $\alpha$ will be invariant with time, a point of fundamental significance to solving the problem of model structure identification. Henceforth in this derivation of the error model, the states and parameters will be treated identically. Although the ultimate goal is the statement of a particular form of recursive estimation algorithm (the extended Kalman filter), in this first stage we shall formulate simply the principles of a general, first-order error analysis. 
Let us assume that it is possible to generate a nominal (deterministic) reference trajectory for the states $\overline{\mathbf{x}}(t)$ (and for the parameters $\bar{\alpha}(t))$ by assuming a set of known initial states $\overline{\mathbf{x}}\left(t_{0}\right)$, known (prior) parameter values $\bar{\alpha}\left(t_{0}\right)$, and a sequence of input disturbances $\overline{\mathbf{u}}(t)$ known for all $t$. The $\overline{\mathbf{x}}(t)$ and $\bar{\alpha}(t)$ can now be generated as (in terms of the augmented stateparameter vector)

$$
d \overline{\mathbf{x}}_{a}(t) / d t=\mathbf{f}_{a}\{\overline{\mathbf{x}}, \overline{\mathbf{u}}, \overline{\boldsymbol{\alpha}} ; t\}
$$

with a correspondingly generated set of "reference" output observations

$$
\overline{\mathbf{y}}\left(t_{k}\right)=\mathbf{h}\left\{\overline{\mathbf{x}}, \bar{\alpha} ; t_{k}\right\}
$$

For the augmented state-parameter vector dynamics (7a), $\mathbf{f}_{a}\{\cdot\}=[\mathbf{f}\{\cdot\} \mid 0]$, which for the simple random-walk model for the parameter variations implies that $\bar{\alpha}$ is a constant for all $t$.

The reference model of (7) is the basis for linearization of the original nonlinear problem. Hence if we introduce the small perturbations (variations) or errors $\tilde{\mathbf{x}}, \tilde{\boldsymbol{\alpha}}, \tilde{\mathbf{u}}$, and $\tilde{\boldsymbol{y}}$ defined as

$$
\tilde{\mathbf{x}}=\mathbf{x}-\overline{\mathbf{x}} \quad \tilde{\mathbf{u}}=\mathbf{u}-\overline{\mathbf{u}} \quad \tilde{\boldsymbol{\alpha}}=\boldsymbol{\alpha}-\overline{\boldsymbol{\alpha}} \quad \tilde{\mathbf{y}}=\mathbf{y}-\overline{\mathbf{y}}
$$

and then take first-order Taylor series approximations of $\mathbf{f}\{\cdot\}$ and $\mathbf{h}\{\cdot\}$ in (6), the nonlinear system model of (6) can be approximated by the combination of the reference model (equation (7)) and the following linear error propagation model (or small perturbations model):

$$
\begin{gathered}
d \tilde{\mathbf{x}}_{a}(t) / d t=F_{a}(t) \tilde{\mathbf{x}}_{a}(t)+G_{a}(t) \tilde{\mathbf{u}}(t)+\xi_{a}(t) \\
\tilde{\mathbf{y}}\left(t_{k}\right)=H\left(t_{k}\right) \tilde{\mathbf{x}}_{a}\left(t_{k}\right)+\eta\left(t_{k}\right)
\end{gathered}
$$

Here $\tilde{\mathbf{x}}_{a}$ is the augmented state-parameter error vector, and $\xi_{a}$ is defined as $[\xi \mid \zeta]^{T}$. The matrices $F_{a}, G_{a}$, and $H$ are appropriate Jacobian matrices (containing derivatives of $\mathbf{f}$ and $\mathbf{h}$ with respect to $\mathbf{x}, \boldsymbol{\alpha}$, and $\mathbf{u}$ ); they derive from the linearization procedure, are in general functions of $\overline{\mathbf{x}}, \overline{\mathbf{u}}$, and $\bar{\alpha}$, and are therefore time varying, as indicated in (9).

The error model can alternatively be written in a discretetime difference equation format by integrating $(9 a)$ over the sampling interval $t_{k-1}$ to $t_{k}$, so that [e.g., Dorf, 1965]

$$
\begin{gathered}
\tilde{\mathbf{x}}_{a}\left(t_{k}\right)=\Phi_{a}\left(t_{k-1}\right) \tilde{\mathbf{x}}_{a}\left(t_{k-1}\right)+\Gamma_{a}\left(t_{k-1}\right) \tilde{\mathbf{u}}\left(t_{k-1}\right)+\xi_{a}\left(t_{k-1}\right) \\
\tilde{\mathbf{y}}\left(t_{k}\right)=H\left(t_{k}\right) \tilde{\mathbf{x}}_{a}\left(t_{k}\right)+\eta\left(t_{k}\right)
\end{gathered}
$$

in which $\xi_{a}\left(t_{k-1}\right)$ is the discrete-time equivalent of the continuous-time noise process $\xi_{a}(t)$.

We have therefore a model for the deterministic reference trajectories of the states and parameters (equation (7)) and a general linear model for the propagation of errors associated with these states and parameters (equation (9)). The reference trajectory can be specified by a suitable choice of $\overline{\mathbf{x}}\left(t_{0}\right), \bar{\alpha}\left(t_{0}\right)$, and $\overline{\mathbf{u}}(t)$ for all $t$. Any errors associated with these choices, together with uncertainty in the unobserved input disturbances of the system $\left(\xi_{a}\right)$, are propagated with time according to $(9 a)$. All these sources of error, together with uncertainties in the observed responses of the system $(\boldsymbol{\eta})$, can be transformed via $(9 b)$ to account for the errors associated with estimates of $\mathbf{y}$ (as opposed to $\mathbf{x}_{a}$ ). Such an error model is applicable, irrespective of whether we are discussing identification, prediction, the extended Kalman filter, or any other estimation scheme.

4.3.2. The extended Kalman filter $(E K F)$. There is a common structure to all recursive estimation algorithms designed for the processing of discrete-time observations of the system's behavior. It breaks down into three components: (1) a prediction step across the sampling interval $t_{k-1} \rightarrow t_{k}$, (2) a correction step at the sampling instant $t_{k}$ as new observations $\mathbf{y}\left(t_{k}\right)$ become available, and (3) a feedback gain matrix (or Kalman gain matrix), which provides a weighting procedure for taking account of the mismatch between the estimate and observed values of the system's response (y).

Each component has its counterpart, roughly speaking, in the nature of the propagation of the various sources of uncertainty.

1. Equation $(10 a)$ of the error model defines the uncertainty associated with the predicted states and parameters at time $t_{k}$ as a function of the errors in the prior ("initial") state and parameter estimates and in the observed and unobserved input disturbances (all at time $t_{k-1}$ ).

2. Equation $(10 b)$ defines the uncertainty of the mismatch between the observed and predicted output responses to be a function of their respective uncertainties.

3. The gain matrix can be chosen such that it minimizes the uncertainty of the corrected state and parameter estimates [Gelb, 1974], and in fact this choice will be seen to involve a "balance" between the two types of uncertainty referred to in point 2 above.

Overall therefore the form of the recursive estimator is such that it embraces a solution to the problem of prediction and prediction error propagation as a part of its structure. This approximate solution, as derived here, is equivalent to a firstorder error analysis; it is a general statement of the propagation with time of the mean and variance-covariance matrix of the state and parameter estimation errors. The additional feature of the estimator is the change made to these uncertainties each time a new observation of the system's behavior is processed. Ideally the change will be a reduction in the uncertainty of the state and parameter estimates, i.e., a reduction in the uncertainty of the internal description of the system's behavior.

The most important differences among the types of recursive estimator to be discussed in this review (the EKF, an instrumental variable (IV), and a least squares (LS) algorithm) lie in their assumed models of the state-parameter dynamics and in the assumed presence (or absence) of the various sources of uncertainty.

The special significance of the EKF for the present discussion is that it refers to a class II form of model; it refers also to the most general formulation of the problem of model structure identification, and it illustrates most completely the way in which uncertainty influences the solution of this problem. There are several routes by which to derive the filter (as discussed in the general texts by Jazwinski [1970], Gelb [1974], and Young [1984], some of which expose its origins in linear, least squares regression analysis and its relationship with the other recursive estimation algorithms to be discussed below [Young, 1984; Beck, 1979b]. The details of these derivations are clearly outside the scope of this review and are not essential to the desired qualitative understanding of the functioning of the algorithms. What we should note, however, is that none of the estimation algorithms was ever developed with the problem of model structure identification in mind.

The estimation problem for our class II model has a high degree of difficulty, being nonlinear, and requiring estimates of quantities (principally the states) that vary with continuous 
time (as opposed to discrete time). The model of the system's behavior has been partitioned into a component assumed to be known with certainty a priori (the nonlinear reference model, equation (7)) and an approximate component into which all the uncertainty has been lumped (the linear error propagation model of (9) and (10)). It would thus be possible to incorporate the linear error model within a linear Kalman filter formulation in order to obtain estimates $\left\langle\tilde{\mathbf{x}}_{a}\left(t \mid t_{k}\right)\right\rangle$ of the errors, or small perturbations in states and parameters, $\tilde{\mathbf{x}}_{a}(t)$ Here the notation $\left(t \mid t_{k}\right)$ indicates an estimate at time $t$ conditioned upon all the input/output observations up to and including those available at $t_{k}$. Hence approximate estimates of the states-parameters $\left\langle\mathbf{x}_{a}\left(t \mid t_{k}\right)\right\rangle$ could be reconstructed using the reference model, i.e.,

$$
\left\langle\mathbf{x}_{a}\left(t \mid t_{k}\right)\right\rangle=\overline{\mathbf{x}}_{a}(t)+\left\langle\tilde{\mathbf{x}}_{a}\left(t \mid t_{k}\right)\right\rangle
$$

Such a reconstruction, however, depends on a good prior choice of the model structure and the values for $\overline{\mathbf{x}}\left(t_{0}\right), \bar{\alpha}\left(t_{0}\right)$, and $\overrightarrow{\mathbf{u}}(t)$ that are used to generate the reference trajectory $\overline{\mathbf{x}}_{a}(t)$. In order to overcome this obvious difficulty two quite specific substitutions are incorporated into the algorithms of the EKF.

1. In the absence of better choices it is sensible to substitute $\overline{\mathbf{u}}(t)=\mathbf{u}\left(t_{k-1}\right)$ for $t_{k-1} \leq t \leq t_{k}$, i.e., to utilize the observed input information to generate the reference trajectory.

2. In order to minimize the possibility of large deviations of the reference trajectory from the true state trajectory it is prudent to "relinearize" at each sampling instant by the substitution of $\overline{\mathbf{x}}_{a}\left(t_{k}\right)=\left\langle\mathbf{x}_{a}\left(t_{k} \mid t_{k}\right)\right\rangle$.

This latter is evidently the means whereby observed information about the system's response (y) is fed back for adaptation of the reference trajectory (paradoxically this might be seen as a disadvantage from the perspective of developing novel and improved algorithms for model structure identification).

To summarize, the qualitative structure of the EKF is as follows. It assumes that the proposed model can be used in a deterministic fashion to generate a reference state-parameter trajectory; all the uncertainties of the internal and external descriptions of the system are lumped into a first-order approximation of error propagation. The propagation of these uncertainties influences the way in which the statesparameters are estimated, and these continuously revised estimates are in turn used to adapt the reference trajectory.

Quantitatively, the extended Kalman filter can thus be stated as (in its continuous-discrete form).

Prediction

$$
\begin{gathered}
\left\langle\mathbf{x}_{a}\left(t_{k} \mid t_{k-1}\right)\right\rangle=\left\langle\mathbf{x}_{a}\left(t_{k-1} \mid t_{k-1}\right)\right\rangle \\
+\int_{t_{k-1}}^{t_{k}} \mathbf{f}_{a}\left\{\left\langle\mathbf{x}\left(t \mid t_{k-1}\right)\right\rangle, \mathbf{u}\left(t_{k-1}\right),\left\langle\boldsymbol{\alpha}\left(t_{k-1} \mid t_{k-1}\right)\right\rangle\right\} d t \\
P_{a}\left(t_{k} \mid t_{k-1}\right)=\Phi_{a} P_{a}\left(t_{k-1} \mid t_{k-1}\right) \Phi_{a}{ }^{T}+Q_{a}
\end{gathered}
$$

Correction

$$
\begin{array}{r}
\left\langle\mathbf{x}_{a}\left(t_{k} \mid t_{k}\right)\right\rangle=\left\langle\mathbf{x}_{a}\left(t_{k} \mid t_{k-1}\right)\right\rangle+K\left(t_{k}\right)\left[\mathbf{y}\left(t_{k}\right)\right. \\
\left.-\mathbf{h}\left\{\left\langle\mathbf{x}\left(t_{k} \mid t_{k-1}\right)\right\rangle,\left\langle\alpha\left(t_{k-1} \mid t_{k-1}\right)\right\rangle\right\}\right] \\
P_{a}\left(t_{k} \mid t_{k}\right)=\left[I-K\left(t_{k}\right) H\right] P_{a}\left(t_{k} \mid t_{k-1}\right)\left[I-K\left(t_{k}\right) H\right]^{T} \\
+K\left(t_{k}\right) R K^{T}\left(t_{k}\right)
\end{array}
$$

\section{Gain matrix}

$$
K\left(t_{k}\right)=P_{a}\left(t_{k} \mid t_{k-1}\right) H^{T}\left[H P_{a}\left(t_{k} \mid t_{k-1}\right) H^{T}+R\right]^{-1}
$$

Here the following assumptions and definitions hold: (1) $P_{a}$ is the variance-covariance matrix of state-parameter estimation errors, (2) $K$ is the Kalman gain matrix, (3) $\xi_{a}\left(t_{k}\right)$ is a zero-mean, white Gaussian sequence with variance-covariance

$$
Q_{a}=\left[\begin{array}{c|c}
Q^{S} & 0 \\
\hline 0 & Q^{P}
\end{array}\right]
$$

in which $Q^{S}$ and $Q^{P}$ are the variance-covariance matrices of the state and parameter disturbances, respectively, (4) $\eta\left(t_{k}\right)$ is a zero-mean, white, Gaussian sequence with variancecovariance $R$, and (5) $\Phi_{a}$ and $H$ refer to the linearized system of (10) and will in general be a function of time (their arguments have been omitted for notational clarity).

4.3.3. Some comments on the filter. There are several points to notice about the EKF. First, any deleterious effects of the first-order linearization are propagated primarily through the variance-covariance recursions of $(12 b)$ and $(12 d)$. Equation $(12 b)$, for example, is derived by setting up the product of the errors $\tilde{\mathbf{x}}_{a} \tilde{\mathbf{x}}_{a}{ }^{T}$ from the linear model of $(10 a)$ and then applying the operation of expectation. The partitioned form of this variance-covariance equation can be likewise formulated from appropriate product terms in $\tilde{\mathbf{x}}$ and $\tilde{\alpha}$ and will be discussed in section 7 .

Second, the sources of uncertainty are represented in the fitler as (recalling Figure 1) (1) the prior uncertainty $P_{a}\left(t_{0} \mid t_{0}\right)$ associated with the state-parameter estimates, (2) the uncertainty $Q_{a}$ associated with $\xi_{a}$, which conceptually covers here prior uncertainty of model structure (if not assumed to be part of $P_{a}\left(t_{0} \mid t_{0}\right)$ ), the uncertainty of the unmeasured input disturbances, and (in this case) the uncertainty in the measured inputs (u), and (3) the uncertainty $R$ associated with the measured output responses.

Prior assumptions about all these quantities are required, together with prior assumptions for the estimates $\left\langle\mathbf{x}_{a}\left(t_{0} \mid t_{0}\right)\right\rangle$.

Third, $(12 c)$, correction of the state-parameter estimates, is fundamental to the functioning of the algorithm. It comprises a correction term that is the product of an error, the residual, one-step-ahead or innovations process error, $v\left(t_{k} \mid t_{k-1}\right)$ :

$$
\mathbf{v}\left(t_{k} \mid t_{k-1}\right)=\mathbf{y}\left(t_{k}\right)-\mathbf{h}\left\{\left\langle\mathbf{x}\left(t_{k} \mid t_{k-1}\right)\right\rangle,\left\langle\boldsymbol{\alpha}\left(t_{k-1} \mid t_{k-1}\right)\right\rangle\right\}
$$

and the gain matrix $K$. Thus the gain matrix weights the account taken of the mismatch between the model and the observations of past behavior. It achieves this, as some reflection on $(12 c)$ will show, by "balancing" the uncertainties of the internal description of the system, namely $P_{a}$, with the uncertainty in the external description of the system, in part $R$. Roughly speaking, if the internal description is not well known, relative to the external description (i.e, relatively accurate observations), relatively large account is taken of the prediction errors and relatively large changes are made to the model parameter estimates. This is intuitively what one would expect, and the converse is true when the model (states, parameters) is "believed" to be accurate and subject to little uncertainty. Beyond this we may note that the gain matrix can be shown to be chosen such that the variance of the estimation errors $\tilde{\mathbf{x}}_{a}\left(t \mid t_{k}\right)$ is minimized [Gelb, 1974] and that the form of $(12 c)$ is equivalent to the gradient algorithms of the hill-climbing, search procedures of numerical optimization in 
general and to stochastic approximation methods in particular [Young, 1984].

Finally, looking at the prediction step of $(12 a)$ and $(12 b)$, it is possible to see how the problem of analyzing prediction error propagation might be viewed as that of applying the EKF to a situation in which the next observation of the system's (future) behavior is to be observed at an infinite time horizon. Alternatively one can think of this as the case where at each future sampling instant the anticipated observations are missing. Both points of view amount to the omission of the correction and gain matrix functions from the algorithm.

Given then the observations of the system's behavior, i.e., $\left\{\mathbf{u}\left(t_{k}\right), \mathbf{y}\left(t_{k}\right)\right\}$ for $k=1, \cdots, N$, we now have an algorithm that can compute recursive estimates $\left\langle\boldsymbol{\alpha}\left(t_{k} \mid t_{k}\right)\right\rangle$ of the model parameter values. However, for the purposes of this paper the real significance of being able to estimate $\left\langle\alpha\left(t_{k} \mid t_{k}\right)\right\rangle$ is not that we can estimate the posterior estimates $\left\langle\alpha\left(t_{N} \mid t_{N}\right)\right\rangle$ but that knowledge and interpretation of the variations in these estimates over the interval $t_{1} \rightarrow t_{N}$ can be used for the identification of model structure.

4.3.4. Least squares and instrumental variable algorithms. The EKF takes observed information about the external description of the system and reconstructs information about the system's internal description $\{\mathbf{x}, \boldsymbol{\alpha}\}$ according to the class II model structure of (2). In order to achieve this it requires extensive assumptions about the types and sources of uncertainty affecting both such descriptions, and therein as we shall see, lie serious limitations of the EKF.

The IV algorithm, in the particular form due to Young [1974], requires no such assumptions. In an equivalent fashion it can be said to take information about $\{\mathbf{u}, \mathbf{y}\}$ and, for an input/output class III model (equation (3)), generates estimates of $\left\{\mathbf{x}^{*}, \boldsymbol{\beta}\right\}$, where $\mathbf{x}^{*}$ can be thought of as the instrumental variable vector and $\boldsymbol{\beta}$ is an appropriate vector of model parameters. Caution must be exercised in referring to $\left\{\mathbf{x}^{*}, \boldsymbol{\beta}\right\}$ as part of either the internal or the external description of the system's behavior. In the original motivation for the development of an IV algorithm, to overcome the problem of biased parameter estimates generated by an LS estimator, the requirements of the instrumental variables were that they should be strongly correlated with the hypothetical noise-free output of the system and entirely uncorrelated with the noise processes affecting the system's observed behavior, namely, $\omega$ in (3). In this case $\left\{\mathbf{x}^{*}, \boldsymbol{\beta}\right\}$ would appear to be associated with an (alternative ) expression of the external description of the system's behavior. As such they encapsulate the identified relationship between $\mathbf{u}$ and $\mathbf{y}$ but make no reference to the physical, chemical, or biological phenomena thought to govern that relationship. The limitations of the IV algorithm, if any, stem from the objections that might be raised about such a seemingly superficial model of a system's behavior. It is often said that the parameters $\boldsymbol{\beta}$ of the input/output model have "no physical meaning."

This distinction, however, between the use of the class II model representation for the EKF and a class III model representation for the IV algorithm is important but misleading, if it obscures a proper appreciation of the different ways in which the different assumptions about the sources of uncertainty influence solution of the identification problem. In essence the EKF, IV, and LS estimators achieve much the same objective in much the same algorithmic fashion. This is especially apparent for the not uncommon case in which the outputs $\mathbf{y}$ are simply error-corrupted observations of the states $\mathbf{x}$, and with all states being observed. The instrumental variable vector $\mathbf{x}^{*}$ is then conceptually indistinguishable from a state estimate. Moreover, if the class II model is linear, it can be transformed by integration over the sampling interval $\left(t_{k-1} \rightarrow t_{k}\right)$ to a class III model representation, and indeed the IV algorithm can be shown to be a self-adaptive state estimator [Young, 1979]. In this case, $\boldsymbol{\beta}$ can be related explicitly to the parameter vector $\boldsymbol{\alpha}$ and, insofar as $\boldsymbol{\alpha}$ is believed to be physically meaningful, so too then is $\boldsymbol{\beta}$. Thus for special cases, $\left\{\mathbf{x}^{*}, \boldsymbol{\beta}\right\}$ can be thought of as an internal description of the system's behavior, and deliberate use will be made subsequently of one of the case studies in order to emphasize this point (see also Beck $[1985 c]$ ).

Young's [1974] form of the IV algorithm exploits what is called an auxiliary model of the system in order to generate the instrumental variables. The instrumental variables, as with the state estimates in the EKF, are quantities that must be known in order to estimate the model parameter values. In this sense the IV is conceptually and computationally equivalent to the EKF. The differences between the two algorithms are that the IV assumes that the sole source of uncertainty is uncertainty in the prior parameter estimates (essentially that all values are more or less equally probable) and that, conversely, the instrumental variables (states) are always known perfectly, i.e., with no uncertainty. Likewise, the recursive LS estimator assumes the same sole source of uncertainty, but in contradistinction to the IV estimator, it assumes that information about the states (instrumental variables) can be substituted directly by the observed output responses $\mathbf{y}$ without explicitly accounting for the errors and uncertainties of this substitution.

An exhaustive discussion of the recursive IV and LS estimators is given elsewhere, by Young [1984]. For the present purpose it suffices to conclude with the observation that different assumptions about the sources of uncertainty lead to different forms of estimators and that these assumptions are probably linked to the differences in the robustness of the performances of the different algorithms. It is certainly true that the EKF has conceptual appeal in its completeness and elegance, but it is not as robust in performance as the IV, a point to which we shall return in section 8 .

\subsection{Model Structure Identification: An Organizing Principle}

The model structure to be evaluated is a more or less complex assembly of several (if not many) constituent hypotheses. Acceptance or rejection of the hypothesis that the model as a whole is adequate will therefore be regarded as a by-product of the analysis. It is possible to formulate a test of this aggregate hypothesis in more conventional statistical terms (as, for example, in the work by Schweppe [1973, 1978]), and this would most probably involve some assessment of the properties of the residual errors defined by (13). However, in this review such problems will only be considered insofar as they are part of model-order estimation or model discrimination as defined above (see also section 6).

4.4.1. Exposing the failure of constituent hypotheses. To visualize how this process of failure might occur, let us suppose that the constituent model hypotheses are parameterized through $\boldsymbol{\alpha}$. Suppose further that the state variables $\mathbf{x}$ of the model (6) can be represented by the nodes of Figure $5 b$ and 
(a)

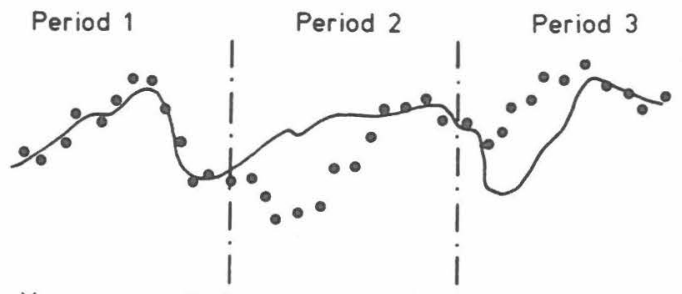

(b)

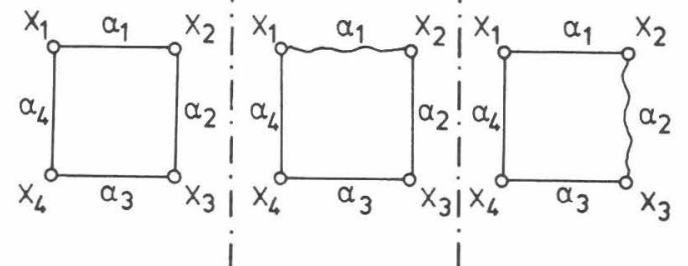

(c)

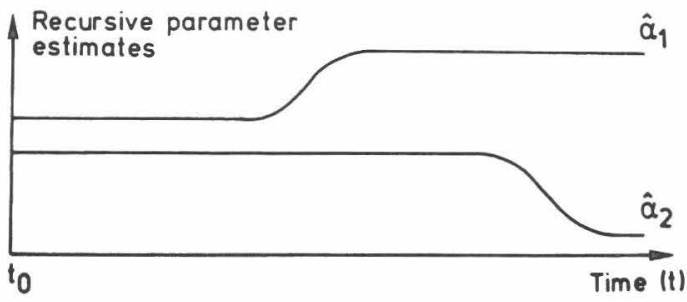

Fig. 5. An illustrative example showing the concept of using a recursive parameter estimator in the context of model structure identification: (a) hypothetical model response with observations (denoted by dots); $(b)$ conceptual picture of model structure; and $(c)$ recursive parameter estimates.

that the parameters $\alpha$ are the branches (or "elastic" connections) between the state variables. The parameters in this simplified sense express the relationships among the system's variables. If now the assumption has been made that all the parameters have values that are constant with time (i.e., $\zeta(t)$ is identically zero in $(6 a)$ ) yet a recursive algorithm yields an estimate of one or more of the parameters that is significantly time varying, one may question as follows the correctness of the chosen model structure. The general tendency of an estimation procedure is to provide estimates $\langle\mathbf{y}\rangle$ of the output responses that track the observations $\mathbf{y}$. Hence if any persistent structural discrepancy is detected between the model and "reality" (in other words, the innovation errors $v$ of (13) exhibit a significantly nonrandom pattern), this will be revealed in terms of significant adaptation of the estimated parameter values. There may well be good reasons why the parameter estimates vary with time, and indeed, that is precisely what one is looking for.

Over period 1 of the example in Figure $5 a$ the model responses $\langle\mathbf{y}\rangle$ and output observations $\mathbf{y}$ are essentially in agreement, and there is no significant adaptation of the parameter estimates (according to Figure $5 c$ ). At the beginning of period 2, however, there is a persistent discrepancy between $\langle\mathbf{y}\rangle$ and $\mathbf{y}$. It might be supposed, for example, that the underlying cause of the discrepancy is an inadequacy in the behavior simulated for $x_{1}$ and $x_{2}$, that $\alpha_{1}$ is sensitive to this discrepancy (Figure $5 b$ ), and that (persistent) adaptation of the estimate $\left\langle\alpha_{1}\right\rangle$ (Figure $5 c$ ) partly compensates for the error between $\langle\mathbf{y}\rangle$ and $\mathbf{y}$. Again in the third period there is disagreement between the observations and model responses, which leads to adaptation of the estimate $\left\langle\alpha_{2}\right\rangle$.

In a qualitative, nonrigorous sense it is these changes of the estimates of unknown but constant parameters that are symp- toms of the failure of individual hypotheses [Beck and Young, 1976; Beck, 1979a, 1983]. By analogy with physical engineering structures, there has been a plastic deformation, or collapse, of a structural member (component hypothesis). Intuitively, such an interpretation, and its similarities with a Popperian view of the scientific method (section 1.2), has considerable appeal. Indeed, this has practical significance for the implementation of the EKF in particular and profound implications for the assumptions made about the uncertainty associated with the prior model hypotheses (and therefore the model structure). If one seeks to expose the failure of hypotheses in this way, it is arguably inconsistent to assume a priori that the parameters $\alpha$ are not only uncertain but are also variable with time. This would be tantamount to seeking the collapse of an extremely "flexible" structure, and clear-cut answers to the identification of model structure would not be generated because, in effect, clear-cut questions are not being asked. So as a practical computational consequence of this philosophical position, the assumption that $\zeta(t)=\mathbf{0}$ for all $t$ in the model for the parameter dynamics, and thus that $Q^{P}=0$, is crucial to exposing the failure of hypotheses.

The prior assumption is that $d \boldsymbol{\alpha}(t) / d t=\mathbf{0}$, i.e., the parameters are truly constant, with the expectation of the posterior result that $d\langle\boldsymbol{\alpha}(t)\rangle / d t \neq \mathbf{0}$, i.e., the parameter estimates vary significantly, thus denying the prior assumption and revealing the failure of a constituent hypothesis.

4.4.2. Further speculation. To be able to demonstrate the inadequacy of a model structure is one thing. To be able to draw inference about how to restructure the model in order to eliminate the cause of the inadequacy is quite another, but it can still in part be accommodated within the framework of using recursive estimation algorithms.

Consider therefore Figure $6 a$, and let us suppose that the foregoing steps in the identification of the model structure have exposed the failure of the hypothesis relating state $x_{2}$ to $x_{3}$. The assumption that $\zeta(t) \neq 0$, and hence that $Q^{P}>0$, now becomes equally crucial in assisting and prompting speculation about possible revised model structures. Given this prior assumption, that the parameters vary in an unknown, random-walk fashion, the expectation of the posterior result is that a more insightful model for the estimated parameter variations can be postulated, for example, in this case,

$$
\left\langle\alpha_{2}(t)\right\rangle=g\left\{\mathbf{x}^{\prime}(t), \boldsymbol{\alpha}^{\prime}, \mathbf{u}(t)\right\}
$$

in which $\mathbf{x}^{\prime}$ and $\boldsymbol{\alpha}^{\prime}$ are possibly revised definitions of the model's state and parameter vectors. In other words, one is looking for a correlation between the estimated variations in $\alpha_{2}$ (when deliberately created as a random walk) and observed or explicable variations in another dependent variable. The ultimate objective would be to interpret the estimated variations in the prior model parameter estimates in some meaningful manner and hence replace an inadequate model structure with essentially time-varying prior parameters $\alpha(t)$ by a structure with essentially constant posterior parameters $\alpha^{\prime}$. In our hypothetical example the structure of Figure $6 b$ might be such a posterior description of observed behavior, so that when subjected to the preceding test for the failure of hypotheses, it survives, yielding parameter estimates with no significant temporal variability.

Of course, the immediate problem in this case is the need to specify $Q^{P}$ so that the potential for the prior model structure to reveal the roots of its inadequacy can be intelligently 
(a)

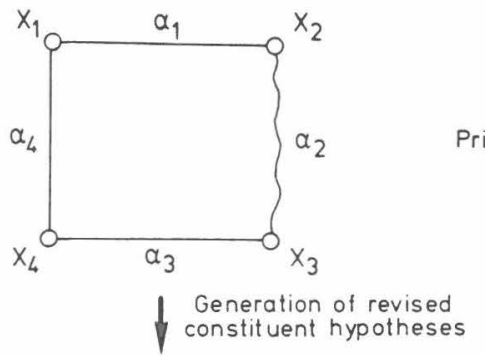

(b)

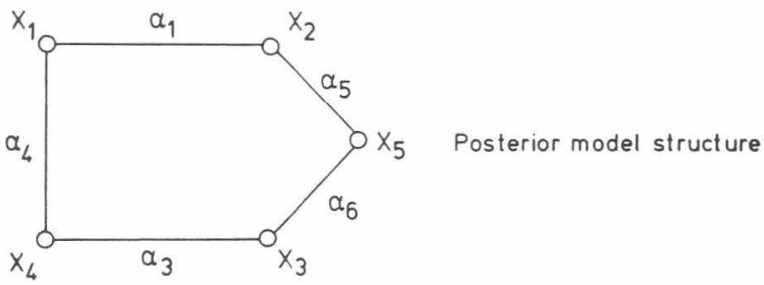

Fig. 6. The process of model structure identification: revision of the model structure and re-estimation of the associated parameters $(b)$ on the basis of diagnosing how the prior model structure fails (a).

probed. Suppose therefore that a set of estimates $\left\langle\boldsymbol{\alpha}\left(t_{N}\right)\right\rangle$ has been obtained for this model structure under the assumption that $Q^{P}=0$. Suppose further that the a posteriori parameter estimation error variance-covariance matrix $P^{P}\left(t_{N}\right)$ has also been determined. Given $\left\langle\boldsymbol{\alpha}\left(t_{N}\right)\right\rangle$ and $P^{P}\left(t_{N}\right)$, a final pass through the set of observations over $t_{0} \rightarrow t_{N}$ could then be made in which $\alpha$ is assumed to be subject to perturbations $\zeta$ with a variance-covariance structure proportional in some way to $P^{P}\left(t_{N}\right)$, e.g.,

$$
Q^{P}=\lambda P^{P}\left(t_{N}\right)
$$

in which $\lambda$ is a scalar [Young, 1974, 1984]. Hence those parameters that have been poorly estimated and are associated with the larger elements of $P^{P}\left(t_{N}\right)$-in part, possibly the prior assumption of truly constant parameters is invalid-would have a higher probability of having estimates that exhibit considerable variation with time. Conversely, those parameters that have been well estimated are unlikely to yield significantly varying estimates. To summarize, such an analysis is tantamount to an evaluation of the appropriateness of the identified model structure. The analysis is, as it were, a controlled speculation using $P^{P}\left(t_{N}\right)$ as a synopsis of the residual uncertainty of identification; in this it is conceptually similar to the HSY algorithm discussed in section 3. Any latitude afforded by $P^{P}\left(t_{N}\right)$ will be exploited in the event of a significantly nonrandom mismatch between the model structure and the observed behavior. Defining $Q^{P}$ according to (15) has much to recommend it, including the fact that the only arbitrary assumption is the choice of a value for the scalar $\lambda$. However, (15) does depend upon a reliable estimate of $P^{P}\left(t_{N}\right)$, which though possible for the IV algorithm, is dubious in the case of the EKF as presently formulated.

So we see that the selection and evaluation of a model structure are iterative processes, alternately seeking to falsify confidently stated hypotheses and then attempting to speculate about relatively uncertain (but improved) hypotheses. The process might be initiated by an analysis of the form discussed in section 3 (generating preliminary hypotheses; see also Whitehead and Hornberger [1984]) and would ideally need to be terminated by an appropriate stopping rule. Here again, the definition of a quantitative measure of an "adequate" model structure is elusive, other than the qualitative discussion of Figure $6 b$ and the residual error analysis discussed in section 6 . There is, in short, no readily apparent equivalent of Akaike's information criterion or the other measures of adequacy applied to the problem of model-order estimation [Young et al., 1980; Hipel, 1981].

\subsection{Case Studies}

Much of what has been said above has considerable appeal in principle. In practice, however, these procedural steps for model structure identification have many limitations.

Perhaps as for all good problems, the interest in model structure identification arose unintentionally, in this instance from a seemingly straightforward case study in "verifying" a model for dissolved oxygen/biochemical oxygen demand (DOBOD) interactions in the River Cam, England [Beck and Young, 1976; Beck, 1983]. It is only with hindsight that the problem of model structure identification has been defined as such, and the superstructure of the procedural steps using recursive estimation algorithms imposed. These steps, developed largely as they were from the Cam study, can be seen to work relatively well on that example. The argument is clearly circular, and there are other, more advanced case studies, for example, of DO-BOD-algae interactions in the Bedford Ouse River in England, where the approach itself fails [Beck, 1982, 1983]. In fact in this latter the approach fails because of an inability to determine unambiguously where the constituent model hypotheses can be said to have failed and because of the difficulty of absorbing and interpreting the sheer volume of extensive evidence on how the model structure might be improved.

4.5.1. Failure and speculation in the case of Lake Balaton. Some of these difficulties stem from the manifest limitations of the EKF. These, and the relative advantages of the IV algorithm, will be evident in the following discussion of a case study of wind-induced sediment resuspension in Lake Balaton, Hungary (see also Beck [1985b, c], Somlyody [1986], and Pinter et al. [1987]). A simple model of the relevant processes, and one which can be derived straightforwardly from a class I representation [Somlyody, 1980], is given by

$$
\begin{gathered}
d x(t) / d t=-f x(t)+g u(t)+\xi(t) \\
y\left(t_{k}\right)=x\left(t_{k}\right)+h+\eta\left(t_{k}\right)
\end{gathered}
$$

where $x$ is the depth-averaged suspended solids (SS) concentration at a point location in the lake, $u$ is the input wind velocity, $f$ is a parameter associated with particle settling rates, and $g$ is a parameter associated with particle resuspension mechanisms; $h$ can be viewed as a background concentration of SS, i.e., that observed fraction of SS not influenced by wind disturbances, or possibly as a measure of the phytoplankton population concentration, which would also be observed as suspended particulate matter. Collectively, $\xi, \eta$, and $h$ can be thought of as representing the possible effects of all the many other factors that might influence the SS dynamics but are not represented explicitly in the model of (16). The model can be straightforwardly incorporated into the EKF algorithm of (12) and, given that we are seeking to expose the failure of the constituent prior hypotheses of this model, with $f, g$, and $h$ specified as parameters that are constant but unknown.

The observed time series comprise (among other variables) 


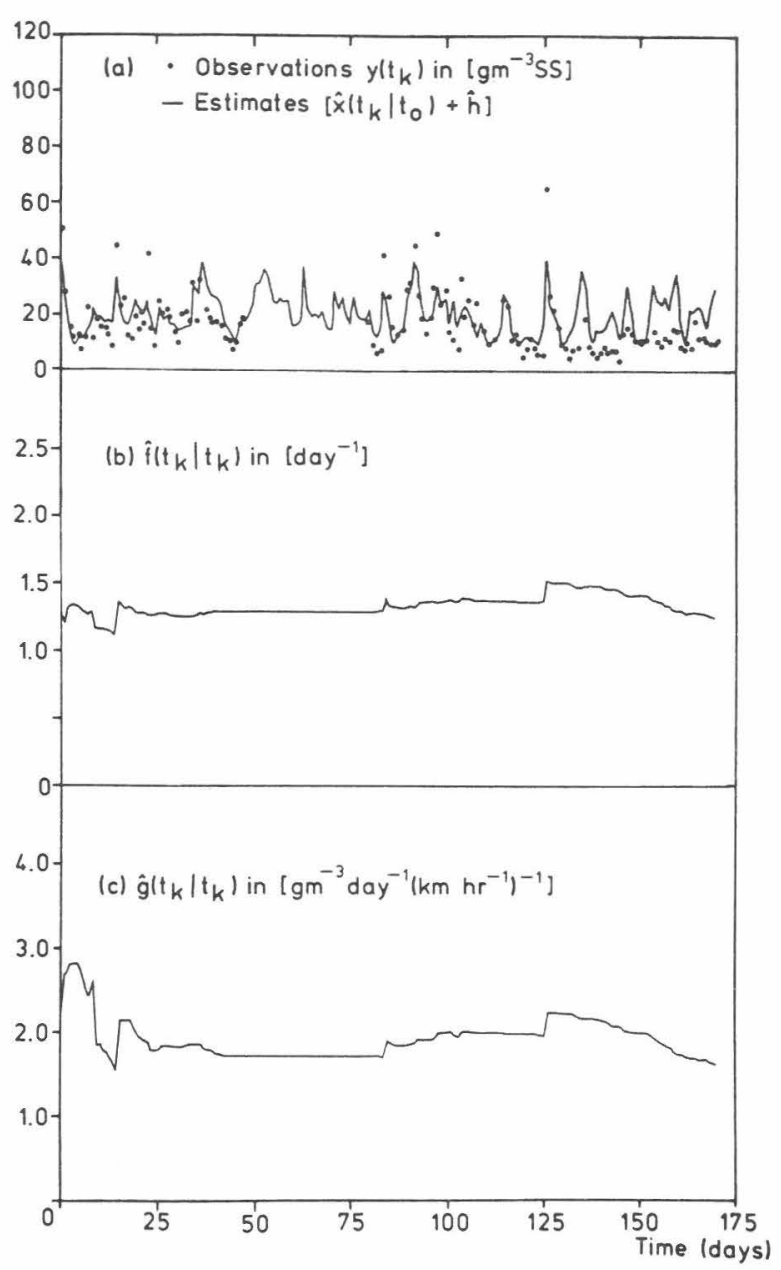

Fig. 7. Recursive state and parameter estimates generated by an extended Kalman filter for sediment resuspension in Lake Balaton.

hourly observations of the wind velocity $u$, and its direction, and daily observations $y$ of the SS concentration (in fact, daily observations at five depths in the water column are available). They cover the period May 14, 1979, to October 31, 1979. The results of Figure 7 are derived from the EKF and are for the case where an estimate $\langle h\rangle=4.0\left(\mathrm{~g} \mathrm{~m}^{-3} \mathrm{SS}\right)$ has been chosen a priori and not included in the state-parameter vector for estimation purposes. Figure $7 a$ shows a comparison of the observed output $y\left(t_{k}\right)$ with the deterministic model response $\left[\left\langle x\left(t_{k} \mid t_{0}\right)\right\rangle+\langle h\rangle\right]$. Figures $7 b$ and $7 c$ show the recursive trajectories of the parameter estimates $\left\langle f\left(t_{k} \mid t_{k}\right)\right\rangle$ and $\left\langle g\left(t_{k} \mid t_{k}\right)\right\rangle$, respectively.

There are three salient features in these results. The parameter estimates fluctuate in a transient manner before settling out by about $t_{25}$; the period of missing observations is a period of summer vacation, although the record of wind velocities is still complete, and most important of all, over the final 45 days of the record the parameter estimates show a significant drift and the observed concentration of SS is persistently overestimated. This "drift" is indeed "significant," for it occurs at a time when the gain matrix $K$ of the EKF is likely to be relatively small, with all but the largest and most persistent errors of mismatch between the model and observations being "ignored" (see the discussion of (12e)). On the basis of Figures $7 b$ and $7 c$ it is difficult to distinguish which of the hypotheses for the two mechanisms (sedimentation or resuspension) is the more inadequate. Over the final $1 \frac{1}{2}$ months (SeptemberOctober) both associated parameter estimates exhibit the nonstationarity that is an essential indication of the failure of a component of the model structure. Overall, the model can be said to fail because during this period the sediment particles appear to be more difficult to resuspend. Looking back, Figure 5 is the conceptual ideal toward which one should be working; Figure 7 is more typical of what will arise in practice with the presently available algorithms.

There now has to be further speculation about why the prior model structure fails. One among at least two competing hypotheses is that the direction of the wind (and not merely its absolute velocity) is important: wind direction affects fetch length, which influences wave action, shear stresses at the water and bottom surfaces, and hence the turbulent vertical movement of water and particles. Noting therefore that the dimensions of Lake Balaton $(70 \times 10 \mathrm{~km})$ give it an elongated shape along a northeast-southwest axis, it turns out that in line with (14) the term $g u(t)$ in $(16 a)$ can be substituted by

$$
g u(t)=g^{\prime}\left|\sin u_{2}(t)\right| u_{1}(t)
$$

to give improved performance (in terms of smaller residual errors). Here $u_{1}(t)$ is (as before) the wind velocity, and $u_{2}$ is the angle between the direction of the wind and the principal axis of the lake.

This is progress - a clue to the roots of the inadequacyalthough the importance of the transversal component of the wind is not consistent with the more obvious ways in which sediment resuspension might be expected to be a function of fetch length and wind direction. Further progress is possible, in particular, using a class III model representation and an IV estimator. If $(16 a)$ is integrated over the interval $t_{k-1} \rightarrow t_{k}$, then (by comparison with the integration of $(9 a)$ to $(10 a)$ )

$$
x\left(t_{k}\right)=\phi x\left(t_{k-1}\right)+\gamma u^{*}\left(t_{k-1}\right)+\xi\left(t_{k-1}\right)
$$

where $u^{*}$ is now the modulus of the transversal component of the wind velocity (thus incorporating the modified hypothesis of (17)). Substituting for $x\left(t_{k}\right)$ from (18) into $(16 b)$, we obtain

$$
y\left(t_{k}\right)=\phi x\left(t_{k-1}\right)+\gamma u^{*}\left(t_{k-1}\right)+h+\left[\xi\left(t_{k-1}\right)+\eta\left(t_{k}\right)\right]
$$

which closely approximates the corresponding class III model (equation (3)) given by

$$
y\left(t_{k}\right)=\phi^{\prime} y\left(t_{k-1}\right)+\gamma^{\prime} u^{*}\left(t_{k-1}\right)+h^{\prime}+\omega\left(t_{k-1}\right)
$$

where $\omega$ is a single lumped-noise process covering the two sources of uncertainty $\xi$ and $\eta$ of (19). The structural equivalence between (19) and (20) would in fact be exact but for the presence of the bias term $h$ in $(16 b)$. Nevertheless, notice that we have an input/output model whose parameters $\left[\phi^{\prime}, \gamma^{\prime}, h^{\prime}\right]$ are essentially similar to parameters that themselves can be related back to physically meaningful quantities.

If a random-walk model is assumed for the possible variations with time of $\left[\phi^{\prime}, \gamma^{\prime}, h^{\prime}\right]$, where the expected variability (uncertainty) of these parameters is specified according to the discussion of (15) in section 4.4., the model of (20) can be usefully employed to probe the structural inadequacies of our prior hypotheses. Moreover, it is both feasible and desirable to consider not the analysis of the depth-averaged SS concentrations but rather an analysis of the response of the system at each of the five spatial points in the vertical to which the original time series refer. In other words, the input/output model of (20) can be applied to each of five combi- 


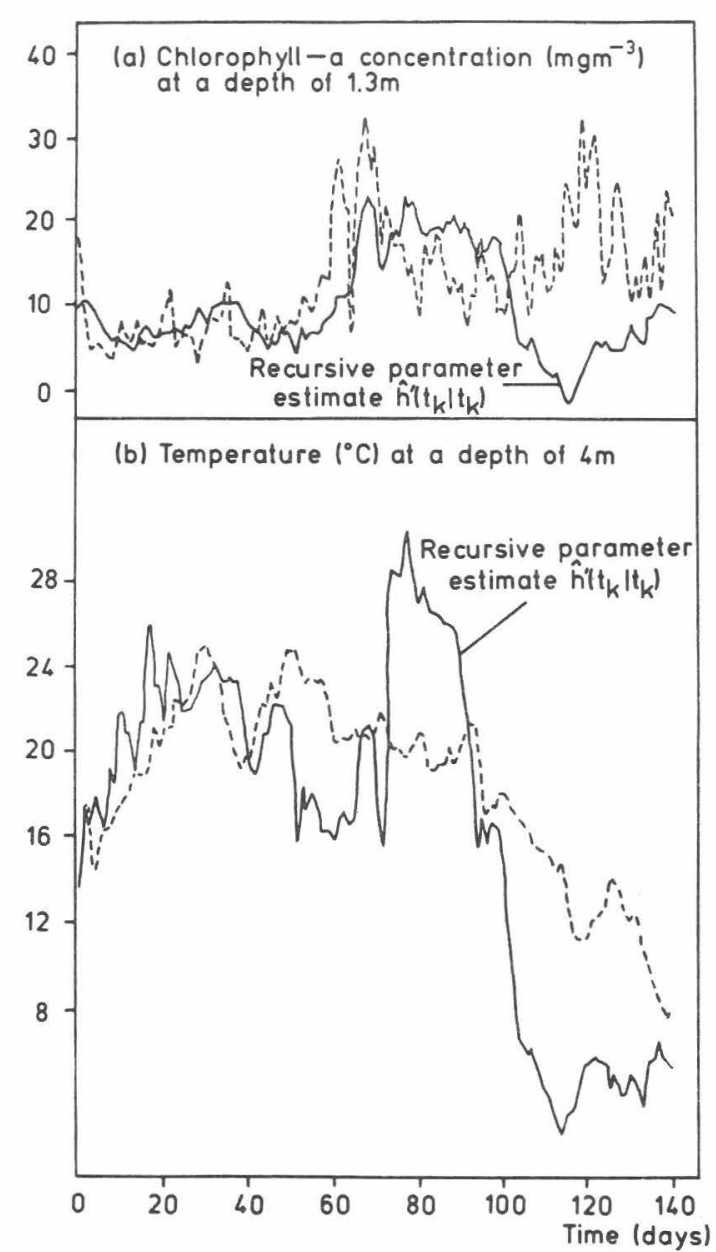

Fig. 8. Comparison of recursive parameter estimates for $h^{\prime}$ in (20) with observed time series (dashed lines) for chlorophyll- $a$ concentration and temperature in Lake Balaton (the estimates have been estimated by a recursive, dynamic least squares algorithm).

nations of the wind input $u^{*}$ and observed SS concentration $y$ at a particular depth in the water column.

It emerges that the parameter representing the background concentration of SS, $h^{\prime}$ in (20), is apparently poorly estimated, varies with time, and is correlated with contemporaneous observations of temperature and chlorophyll $a$ (phytoplankton) concentration [Chan, 1984]. Furthermore, the functional relationship with temperature and/or chlorophyll a concentration is depth dependent. For instance, Figure 8 shows the trajectory of the recursive estimates $\left\langle h^{\prime}\left(t_{k} \mid t_{k}\right)\right\rangle$ derived from the analysis of the SS concentration at a depth of $1.3 \mathrm{~m}$ below the water surface. (The estimates are generated by a dynamic least squares algorithm but within the general framework of IV estimation [e.g., Young, 1984].) It is certainly tempting to speculate that these estimated variations of $\left\langle h^{\prime}\right\rangle$ are correlated with the observed variations in chlorophyll $a$, at least for the first two-thirds of the record, and that this would be consistent with the growth of a population of phytoplankton in the upper layers of the water column, where access to solar radiation is greater (the missing period of observations of Figure $7 a$ has simply been omitted from the plots of Figure 8). We have already alluded to the fact that the SS measurement will be influenced by particulate phytoplankton matter. At the lowest depth at which observations were taken (at $4 \mathrm{~m}$, where the depth of the lake bed is $4.3 \mathrm{~m}$ ), a different pattern for the corresponding estimates $\left\langle h^{\prime}\right\rangle$ is apparent (Figure $8 b$ ). Here the overall trend in $\left\langle h^{\prime}\right\rangle$ is correlated with seasonal variations in temperature, albeit rather tenuously and with obvious anomalies. Such a relationship is not at all straightforward to explain. One can construct a simple argument about temperature influencing the viscosity of water and hence the movment of a particle through the water. But this argument runs counter to what is observed, and it might equally well be that the seasonal variation in $\left\langle h^{\prime}\right\rangle$ is a function of day length and its apparent influence on the diurnal pattern of wind events.

Suffice it to say that a simple modification of (20) to include the effects of temperature variations improves marginally the performance of the model (accounting for chlorophyll $a$ variations is, conversely, not a success). To summarize the case study, the identification of the importance of wind direction, temperature, and chlorophyll $a$ were all to some extent unexpected, and we are still distant from a rigorous and coherent explanation of why this environmental system behaved as it did. For this part of the analysis too, Figure 6 is an ideal: we have merely begun to amass the diagnostic evidence with which to make the required step from Figure $6 a$ to Figure $6 b$.

The key point about the Balaton example is that its results are typical: they are not entirely crisp and clear-cut; but the analysis has yielded a rich source of anomalies, some of which can be partly explained; and it emphasizes the role of models - including a class III representation - as vehicles for the exhaustive interpretation of field data.

4.5.2. Other studies. If anything, there are on balance more difficulties in the design of algorithms that perform well in exposing the failure of constituent model hypotheses. Somewhat more encouraging results have been obtained for the second step of the procedure, i.e., speculation. For instance, Figure 9 shows a comparison of the trajectory of the recursive estimate $\left\langle\alpha\left(t_{k} \mid t_{k}\right)\right\rangle$ for the net rate of addition of BOD in a

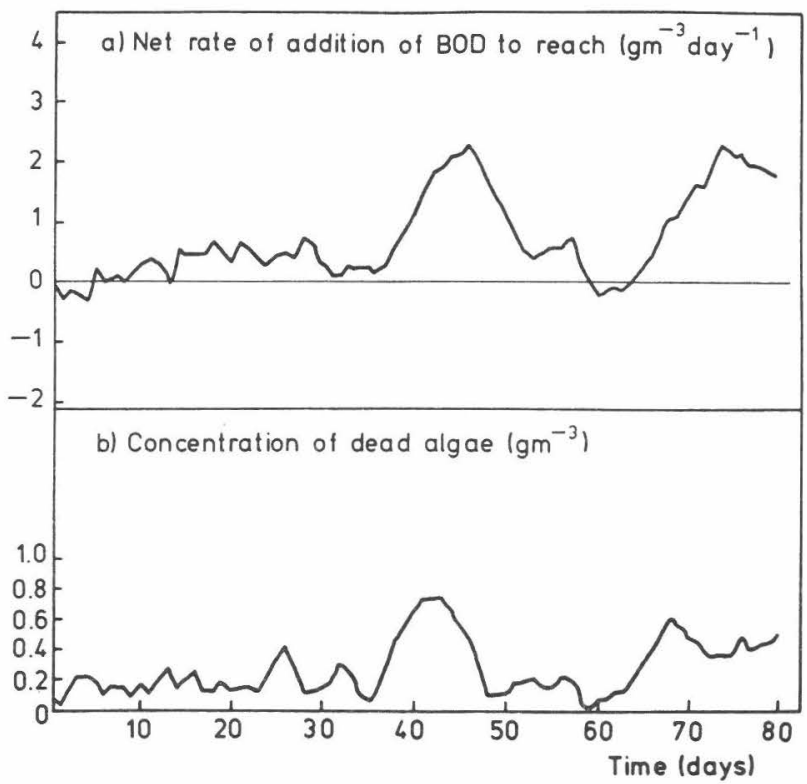

Fig. 9. Results for model structure identification from a case study of the River Cam: $(a)$ recursive parameter estimates $\left\langle\alpha\left(t_{k} \mid t_{k}\right)\right\rangle$, using an extended Kalman filter, for the net rate of addition of BOD to a reach of the river, and $(b)$ estimated variations of a hypothetical state variable representing the concentration of dead algae in the reach. 


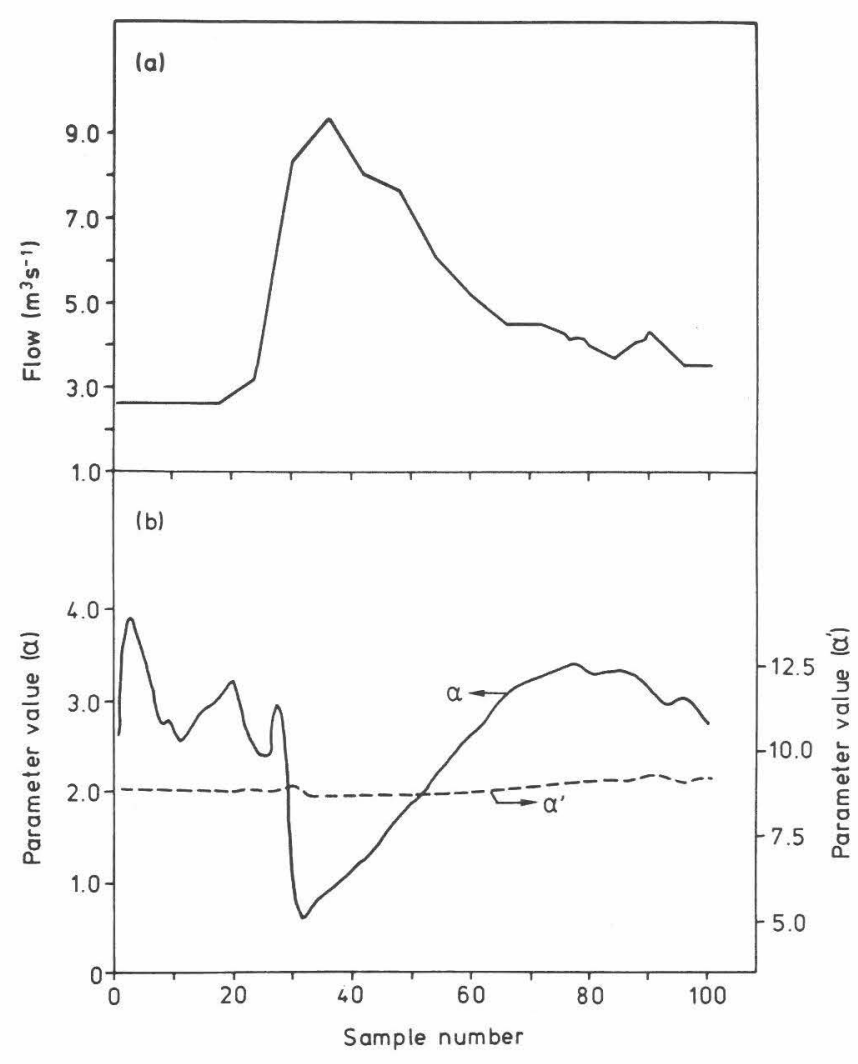

Fig. 10. Results for model structure identification from a case study of the Bedford Ouse River [after Whitehead, 1983]. (a) Streamflow rate. (b) Recursive parameter estimates for a first-order decayrate constant for ammonium $\mathrm{N}$ as in $(21) ;\left\langle\alpha\left(t_{k} \mid t_{k}\right)\right\rangle$ is represented by the solid line, and $\left\langle\alpha^{\prime}\left(t_{k} \mid t_{k}\right)\right\rangle$ by the dashed line (estimates generated by an extended Kalman filter).

reach of river (the Cam) with the simulated variations of a hypothetical state of the system $\left\langle x\left(t_{k}\right)\right\rangle$ defined to be the concentration of dead algal material in the river [from Beck, 1983]. Here $\left\langle x\left(t_{k}\right)\right\rangle$ has been generated as a deterministic function of the observed hours of incident sunlight (as an input, $\left.u\left(t_{k}\right)\right)$. The speculation to be included in a revised posterior model structure, as a consequence of the analysis of the prior model structure, is that there is an addition of BOD in the reach of river whose rate is linearly proportional to the hypothetical concentration of dead algal material. A second example, also using the EKF, is illustated in Figure 10 and is taken from Whitehead's [1983] study of nitrification dynamics in the Bedford Ouse River. In this case, the estimated variations of a first-order rate constant $\left\langle\alpha\left(t_{k} \mid t_{k}\right)\right\rangle$ for the conversion of ammonium $\mathrm{N}$ to nitrite $\mathrm{N}$ and ultimately to nitrate $\mathrm{N}$ are clearly inversely related to the variations of stream discharge $u\left(t_{k}\right)$. The associated speculation that the rate of conversion of ammonium $\mathrm{N}$ can be modified as (in line with (14))

$$
\alpha(t)=\alpha^{\prime} / u(t)
$$

leads, in a revised posterior model structure, to recursive estimates $\left\langle\alpha^{\prime}\left(t_{k} \mid t_{k}\right)\right\rangle$ that are sensibly stationary (see Figure $10 b$ ). For a third example, Figure 11 has been presented by Scavia [1980] in a study of eutrophication in Saginaw Bay, Lake Huron, in the United States. It shows the recursive estimates from an EKF algorithm for the variations of a parameter characterizing the phytoplankton phosphorus-to-chlorophyll ratio and observed values of the ratios of phytoplankton dry weight to chlorophyll. Among other factors, Scavia has speculated that the variable parameter estimate may reflect a sequence of changes in the dominant observed phytoplankton groups, as also shown in Figure 11. A similar speculation has been offered by Whitehead and Hornberger [1984] in their study of modeling algal population dynamics in the River Thames, England.

There are several other applications of the EKF reported in the literature on the modeling of water quality ecological systems, although most of these, for example, Lettenmaier and Burges [1976] (an introductory paper), Bowles and Grenney [1978a, b], Constable and McBean [1979], and Whitehead et al. [1981], deal with the more conventional problem of parameter estimation (as opposed to model structure identification). Among these, Bowles and Grenney's [1978a] study of nitrogen-cycle kinetics in the Jordan River, Utah, offers useful comments both on several minor extensions of the basic algorithm and on practical limitations to the performance of the EKF. Cosby and Hornberger [1984] and Cosby et al. [1984] have recently reported a study on the application of the EKF to a problem of discriminating among competing model structures for the description of nonlinear light-photosynthesis relationships in a freshwater stream. We shall, however, defer further discussion of this type of problem until section 6. Two other papers, by Koivo and Phillips [1976] and Tamura [1979], though they do not deal with the analysis of in situ field data, are notable from the point of view of problem formulations leading to the use of a linear Kalman filter. Tamura, for example, formulates the problem of parameter estimation as if it were a simplified problem of pure state estimation for a system whose state is time invariant. Indeed, the form of duality between state and parameter estimation that this suggests may have especially important implications for the development of novel algorithms for model structure identification (as discussed in section 8 and in the work by Beck [1985c]).

The IV algorithm has been less widely applied than the EKF, most probably because of its usual association with an input/output class III model representation and the objections raised against such models with parameters said to be not physically meaningful. The discussion of wind-induced sediment resuspension, however, should have countered some of

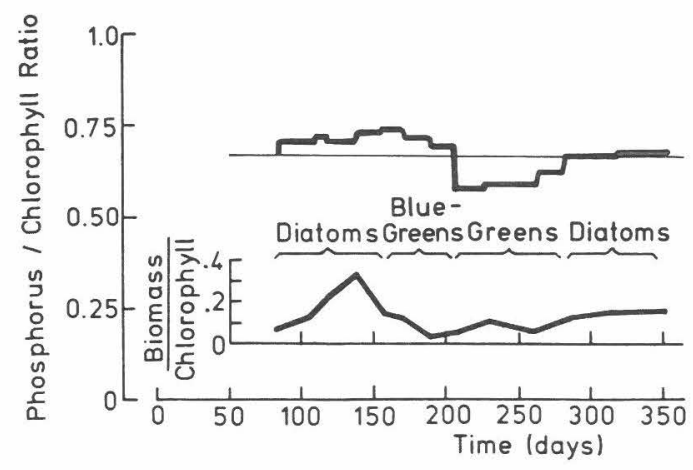

Fig. 11. Results for model structure identification from a case study of Saginaw Bay, Lake Huron [after Scavia, 1980]. Recursive parameter estimates for a phytoplankton phosphorus to chlorophyll ratio (upper plot) are compared with measurements of phytoplankton dry weight to chlorophyll ratios from Bierman et al. [1980] (estimates generated by an extended Kalman filter). 
these objections. The algorithm is well worth closer attention, perhaps best approached (particularly for hydrological and environmental systems) via the introductory text of Young [1984]. It is not restricted to multiple-input/single-output model forms, although these have been its most common area of application. A version of the IV algorithm suitable for multiple-input/multiple-output models has been proposed by Young and Whitehead [1977] for the previously discussed case studies of DO-BOD-algae interaction in the Rivers Cam and Bedford Ouse (see also Whitehead and Young [1975], Young [1978], and Whitehead [1979]). Another version, appropriate to a class II representation, has been illustrated by Whitehead [1980], again for the Cam example.

The IV algorithm is by no means the only estimator suitable for the classical input/output models of time-series analysis; there are several others [e.g., Åström and Bohlin, 1966; Box and Jenkins, 1970; Åström and Eykhoff, 1971; Eykhoff, 1974], most of which can be written in recursive form [Söderström et al., 1978]. Gentil [1984], for instance, has reported an interesting example for the analysis of phytoplankton-zooplankton dynamics in Lake Aiguebelette, France, using a recursive version of a maximum likelihood estimator. In this case the input/output variables are in fact what we have referred to as the small perturbations in a model similar to (10) and representing perturbations about a nonlinear reference class II model structure (such as (7)). In the event, Gentil concludes from the invariance of her recursive parameter estimates that the prior model structure is adequate (although it is not clear under what precise conditions these estimates have been generated).

\subsection{Commentary}

No analysis of real field data is ever neat, elegant, or straightforward in practice. There is no panacea for the problems of model structure identification and no easy substitute for the painstaking piecing together and sifting of evidence on the rejection and generation of model hypotheses. It is notable that few, if any, rigorous, procedural steps have been introduced in this section, except for the duality of testing a prior model structure to the point of failure and then attempting to infer the form of an improved model structure from the failure of that inadequate prior structure. Even the superstructure of how to employ recursive estimation algorithms that has been built upon this single, simple duality is in danger of becoming overly complicated and convoluted. Something more straightforward would be highly desirable, without denying the latent contribution that the development of such a superstructure has made to the whole of this review. Recursive estimation algorithms provide a unique type of information (on the temporal variability of the model parameters) that is useful in solving the problem of model structure selection and evaluation. However, they are not the only means of solving this problem.

Of the methods, the EKF is certainly not without either its critics or serious limitations. But it has to be said that there are few practical alternatives for the identification of multivariable (class II) model structures, and as an engineering solution to a commonly occurring problem of nonlinear state estimation, the EKF was never intended to satisfy the demands of model structure identification. Its most important limitations are given below.

1. The performance of the algorithm is determined largely by the evolution of the gain matrix $K$, which itself is determined, indeed "prejudiced," by the prior choices of $Q_{a}$, $P_{a}\left(t_{0} \mid t_{0}\right)$, and $R$, and these choices, especially for $Q_{a}$, are more or less arbitrary.

2. It is difficult to distinguish what constitutes a significant variation in a parameter estimate, although one should not exclude the role of expert knowledge of the system from this process. In the particular results of Figure 7 the large initial variations in the parameter estimates have been assigned to transient fluctuations due to the algorithm, while the drifts at the end of the record, where the gain matrix $K$ is relatively small, are supposed to be due to an important mismatch between the model structure and the data.

3. It takes no account of the sample statistics of the innovations, $\mathbf{v}\left(t_{k} \mid t_{k-1}\right)$, that accrue as the algorithm sequentially processes the observations, a point that underlines the dominant influence of prior assumptions on the performance of the algorithm.

4. Because of the linearization approximation of which the EKF is based, it is not possible to interpret the relevant elements of the matrix $P_{a}\left(t_{k} \mid t_{k}\right)$ as accurate estimates of the parameter estimation error variances.

5. The assumption of normal distributions for all random variables is rarely valid, although not as seriously for the precipitation events that dominate hydrological time-series analysis.

If one were to seek to eliminate some of these limitations, pragmatism would set at least three ideals toward which to aim in the development of new algorithms: (1) a minimum number of prior assumptions, including those required to quantify the various sources of uncertainty, in order to implement the algorithm, (2) a minimum amount of computational effort, and (3) a maximum degree of robustness in the performance of the algorithm.

On all three accounts the IV algorithm can at present claim superiority over the EKF. The purist might protest that these ideals are too pragmatic. But the concern of this review is for methods that perform reliably on extremely difficult sets of data, and experience has shown that certain algorithms with impeccable statistical credentials (for example, good asymptotic convergence and efficiency) have been unable to deliver such performance. It is highly relevant that most of the development of recursive estimation algorithms in control theory has taken place in an environment where model structure identification (as defined here) is not usually seen to be a problem.

The IV algorithm does, however, suffer from two disadvantages, both of which are perhaps more apparent than real. First, it is most usually formulated for discrete-time, difference-equation models, which are not the most natural means of describing the behavior of environmental systems. This alone should not be the cause of any difficulty. It is when the continuous-time, differential-equation model contains nonlinearities, as do most of the models of interest, that the clear links between the discrete-time and continuous-time structure become obscured (see, for example, Gentil [1979] on a study of lake eutrophication). Second, and more philosophical, is the general unease about input/output models with parameters that are not physically meaningful. It is hard to imagine an aspect of water quality modeling with seemingly better defined prior theories and more physically meaningful parameters than the conventional advection-dispersion model of pollutant 
transport in a river. All the more reason therefore for the skeptical reader to join the debate opened by Young [1983], Beer and Young [1983], and Young and Wallis [1986], on the use of input/output class III model representations of longitudinal dispersion in natural streams.

Of the approach, it is fair to conclude that its cardinal points of attempting to falsify confidently stated hypotheses and then engaging in creative speculation with relatively uncertain hypotheses, are both satisfying and reassuringly consistent with a Popperian view of the scientific method. They are satisfying because they illuminate the role of uncertainty in evaluating and selecting a model structure; and Popper's view of the scientific method, perhaps surprisingly, gives direction to the possible development of novel algorithms for model structure identification (about which more will be said in section 8). There are minor caveats, however. For example, the inclusion of unobserved state variables in a model can render quite ineffective the principle of seeking to expose the failure of constituent model hypotheses. The distortion (or collapse) of the model structure, in making it fit the observed data, will tend to be accommodated by significant adaptation of the estimates of these unobserved states, whose variability may or may not be meaningless [Beck, 1983] (see also Fedra [1983] for related discussion).

Above all, it is a variety of perspective and approach that is the key to the identification of model structure: a trading of the computational effort of solving natural formulations of ill-posed problems (for example, a class II model with the EKF) against the analytical effort of transforming these to better posed, if slightly distorted, problems (for example, into a class III model for use with the IV algorithm). The models are vehicles for the exhaustive interpretation of field data, and while they may not be satisfactory end points of this process, the oft-maligned class III representations have a legitimate role to play. Both the Balaton (as here) and Cam examples (as in the work by Beck [1978]) are illustrative of this role.

\section{Parameter Estimation}

Most readers, whether or not they have thought about uncertainty, model structure identification, and prediction error propagation, will be familiar with the notion of model calibration, or parameter estimation. It is this perhaps rather restricted view of the subjects of this paper that lies behind the observations quoted earlier from DeLucia and McBain [1981] at the beginning of section 1.2.

Having selected and evaluated a model structure, we come then to what has been defined as problem area $(\mathrm{P} 2)$ in the introduction.

Uncertainty about the values of the parameters (coefficients) appearing in the identified structure of the dynamic model for the system's behavior.

The solution to this problem will be understood here to involve not merely the generation of accurate estimates for the model parameters but also some quantification of the matrix of a posteriori estimation error variances-covariances. The basic issue is can the unknown, but constant, parameter values of a well-identified model structure be estimated accurately, uniquely, and with as little uncertainty as possible?

It is far beyond the scope of this review to cover the entire field of parameter estimation. The texts by Eykhoff [1974], Young [1984], and to a lesser extent, Jazwinski [1970], Schweppe [1973], and Gelb [1974] provide very good intro- ductions to the subject; the recent books by Ljung and Söderström [1983] and Söderström and Stoica [1983] give a more advanced treatment of the theoretical underpinnings of the subject, especially with respect to recursive estimation algorithms and the input/output class III model structures; and there have also been some important review and tutorial papers, of which those by Åström and Eykhoff [1971], Isermann [1981], and Young [1981] are most relevant to the present discussion. This is, of course, a biased sample of the literature, for it reflects solely the control theoretic view of the subject and, with the possible exception of Young [1984], makes little reference to the undoubtedly substantial contributions from statistics and econometrics. Yet unification of the subject and the cross fertilization of ideas among disciplinary compartments are becoming increasingly evident. The International Federation of Automatic Control (IFAC) has held regular symposia on "Identification and System Parameter Estimation" since 1967. The most recent [Barker and Young, 1985] signalled to a notable extent a reassuring departure from the disciplinary confines of control theory: it included special sessions on identification and estimation in the context of operations research and on statistics/time-series analysis. There is too a reciprocal development: econometricians, in particular, have become well aware of the now ubiquitous Kalman filter, though possibly not for the purposes of parameter estimation [Durbin, 1984; Hendry, 1984; Harvey, 1984].

These changes are encouraging and so are other developments of interest; but some are less encouraging. For instance, on the positive side there is much to be learned from the adjacent fields of biomedicine and pharmacokinetics, particularly in respect of the exactly parallel problems of model identifiability and validation [Godfrey and Distefano, 1985; AlDahan et al., 1985; Flood et al., 1985]. Equally positive is the formal introduction of forecasting as a problem area germane to system identification. Somewhat neutral, but certainly of interest, is the maturity achieved in the conceptual and theoretical framework of instrumental variable estimators; as a class of estimators, they now stand alongside least squares, maximum likelihood, Bayesian, and Kalman filter estimators. Finally, from the point of view of identifying models of environmental systems, it is mildly discouraging to note the increasing emphasis of these IFAC symposia on adaptive (realtime) control and an absence of what can be discerned (by the practitioner) to be radically novel algorithms. True, the peripheral observer might not appreciate the implications of theoretical progress, and we shall have more to say on novel algorithms later in section 8 .

For water quality models the overriding difficulty of parameter estimation is that of a lack of parameter identifiability.

\subsection{Types of Estimator}

There are two important classifications to be made: first, a classification according to the type of estimator, and this, as with the foregoing discussion of model structure identification, is defined by the different assumptions that can be made about the sources of uncertainty affecting the estimation problem, and second, a classification of the different algorithms used to implement a batch estimation scheme (which is essentially a matter of choosing a constrained opimization algorithm; see below). A recursive or a batch scheme could in principle be used for each of the four estimators that follow. But since the former have already been discussed in section 4 and since they do not reflect the vast majority of applications to the problem of parameter estimation, it is convenient to concentrate here 
on batch estimation algorithms. The four estimators are distinguished in an ascending order of the degree of completeness in accounting for the various sources of uncertainty (as in the work by Eykhoff [1974]).

5.1.1. Least squares estimator. No a priori knowledge of the statistical properties of any of the sources of uncertainty is required for LS estimation. The estimates of the augmented state-parameter vector $\mathbf{x}_{a}=[\mathbf{x}, \alpha]^{T}$ are derived by minimizing the sum $J$ of the squared errors (e) between the observed and estimated behavior of the system, i.e.,

$$
J=\sum^{N} \mathbf{e}^{T}\left\{\left\langle\mathbf{x}_{a}\right\rangle\right\} \mathbf{e}\left\{\left\langle\mathbf{x}_{a}\right\rangle\right\}
$$

Here the summation is over the $N$ sets of errors $\mathbf{e}\left(t_{k}\right)$ for all the sampled observation instants $t_{k}$. Equation (22) emphasizes the fact that $\mathbf{e}$ is a function of estimates of both the states and parameters, although in most instances the determination of the minimum value of $J$ will be achieved by adjusting only the values of the (constant) parameters $\langle\boldsymbol{\alpha}\rangle$ and not the values of the states for all the instants $t_{k}$, i.e., $\left\langle\mathbf{x}\left(t_{k}\right)\right\rangle$. The error e may be specified in several ways [Young, 1981], or indeed there may be several component error terms, as discussed below, each referring to errors of mismatch originating from the different sources of uncertainty [Eykhoff, 1974; Schweppe, 1973]. However, in all the cases of practical interest to this review, e will be assumed to contain a single-component term for the error between the observed $(\mathbf{y})$ and estimated $(\langle\mathbf{y}\rangle)$ output response variables. Strictly speaking, for an estimator to be defined as an LS estimator, this error would have to be specified as the deterministic model response error

$$
\mathbf{e}\left(t_{k}\right)=\mathbf{y}\left(t_{k}\right)-\left\langle\mathbf{y}\left(t_{k} \mid t_{0}\right)\right\rangle
$$

In other words, $\left\langle\mathbf{y}\left(t_{k} \mid t_{0}\right)\right\rangle$ is based upon $\left\langle\mathbf{x}\left(t_{k} \mid t_{0}\right)\right\rangle$, an estimate of the state of the system conditioned upon observations of the output response up to and including that available at the beginning of the experimental record, time $t_{0}$.

5.1.2. Weighted least squares $(W L S)$ estimator. A weighted least squares estimator yields estimates $\left\langle\mathbf{x}_{a}\right\rangle$ that minimize the modified squared-error sum $J^{*}$ given by

$$
J^{*}=\sum^{N} \mathbf{e}^{T}\left\{\left\langle\mathbf{x}_{a}\right\rangle\right\} W^{-1} \mathbf{e}\left\{\left\langle\mathbf{x}_{a}\right\rangle\right\}
$$

where $W$, the additional prerequisite, is a weighting matrix chosen (in Schweppe's [1973] terms) according to "engineering judgement." A special case of (24) is that where the variancecovariance matrices of, most commonly, the output measurement error sequence $\eta$ (and, more rarely, the sequence of unknown input disturbances $\xi$ and initial condition errors associated with $\left\langle\mathbf{x}_{a}\left(t_{0}\right)\right\rangle$ as an estimate of $\left.\mathbf{x}_{a}\left(t_{0}\right)\right)$ are assumed to be known. In the special case where $W=R$ is chosen as a weighting matrix ( $R$ being the variance-covariance matrix of the output response measurement errors), the resulting estimator would be called by Eykhoff [1974] a Markov estimator. Note that if the error e were to be specified as a function of the other sources of uncertainty, the variance-covariance matrices $Q^{S}$ and $P^{S}\left(t_{0}\right)$ would need to be used as similar weighting matrices for their corresponding constituent squared-error terms. Here $Q^{S}$ reflects the errors in the state vector equations (the input disturbance uncertainty) and $P^{S}\left(t_{0}\right)$ the variance-covariance matrix of errors in the estimates of the initial state. Clearly, the underlying assumption is that the sources of uncertainty can be characterized simply by their mean and variance-covariance statistics, and almost without exception the means are assumed to be zero.
5.1.3. Maximum likelihood $(M L)$ estimator. The a priori knowledge required for a maximum likelihood estimator is a specification of the probability density function $p\{\mathbf{y} ; \boldsymbol{\alpha}\}$ for the population of observations $\mathbf{y}$ as a function of the unknown (and constant) parameter vector $\alpha$, to which may occasionally be adjoined elements of the unknown initial state of the system $\mathbf{x}\left(t_{0}\right)$ [see Maciejowski, 1980]. This prior knowledge implies knowledge of the probability density functions of the random variable $\boldsymbol{\eta}$ and, less frequently, of $\xi$. Here $\alpha$ is not treated as a random variable; hence $p\{\mathbf{y} ; \boldsymbol{\alpha}\}$ is not a joint probability density function for $\mathbf{y}$ and $\boldsymbol{\alpha}$, nor is it a conditional probability density function. The point is a subtle but important one, for it distinguishes an ML estimator from a Bayesian estimator in that the former does not require an assumption about the prior probability density function of the parameter vector $\boldsymbol{\alpha}$. Given a sample set of field observations, say $\mathbf{y}^{i}$, the likelihood function is specified (a posteriori) as $L\left(\mathbf{y}^{i} ;\langle\boldsymbol{\alpha}\rangle\right)$, where the functional relationship between $\langle\boldsymbol{\alpha}\rangle$ and $\mathbf{y}^{i}$ in $L\left\{\mathbf{y}^{i}\right.$; $\langle\boldsymbol{\alpha}\rangle\}$ is the same as that between $\boldsymbol{\alpha}$ and $\mathbf{y}$ in $p\{\mathbf{y} ; \boldsymbol{\alpha}\}$. The maximum likelihood estimates $\langle\boldsymbol{\alpha}\rangle$ are those values of $\boldsymbol{\alpha}$ that maximize the value of $L$, these being the most likely values of the parameters given $\mathbf{y}^{i}$.

5.1.4. Bayesian estimator. As its name suggests, this estimator is based upon the following form of Bayes' rule,

$$
p\{\boldsymbol{\alpha} \mid \mathbf{y}\}=\frac{p\{\mathbf{y} \mid \boldsymbol{\alpha}\} p\{\boldsymbol{\alpha}\}}{p\{\mathbf{y}\}}
$$

and it requires knowledge a priori of all the probability density functions on the right-hand side of (25). (For simplicity the Bayesian (and ML) estimators have been specified in terms of the parameter vector $\alpha$ only (and not in terms of $\mathbf{x}_{a}$ ).) In particular, $\alpha$ is now considered to be a random variable, and over and above the ML estimator, the Bayesian estimator requires assumptions about its prior probability density function $p\{\boldsymbol{\alpha}\}$. In turn, given a sample set of observations $\mathbf{y}^{i}$ the Bayesian estimator yields a complete description of the posterior distribution of the parameter values, from which, however, it will usually be necessary to make some choice of the best set of estimates $\langle\boldsymbol{\alpha}\rangle$ of $\boldsymbol{\alpha}$. The making of this choice may require further prior assumptions for implementing the various decision rules discussed, for instance, by Schweppe [1973] and Eykhoff [1974].

To take stock of this brief treatment of a difficult subject, it may be helpful to make the following qualitative juxtaposition of the two extreme cases of estimators. The simple LS estimator makes no assumptions about $\alpha$. It merely computes those values of $\alpha$ that minimize an objective (fitting) function. The Bayesian estimator requires complete knowledge of the prior probability density function of $\alpha$ based on knowledge of $\mathbf{y}$ as a random variable and some assumed relationship, i.e., the model, between $\mathbf{y}$ and $\alpha$. It mechanizes the translation of information about the external description of the system (y) into information about the internal description of the system $(\alpha)$ through the particular rule of (25). It can, of course, process the observations $\mathbf{y}$ in a sequential fashion, and in this sense, any recursive estimation algorithm can clearly be viewed as a Bayesianlike estimator.

But here we risk confusion, and there is sufficient cause for confusion in understanding the above classification that is brought about by the following.

It is generally assumed that Gaussian probability density functions hold for all of the relevant random variables and 
stochastic processes, and under this assumption the probability density function $p\{\alpha \mid y\}$ in (25) is specified simply by its mean and variance-covariance matrix. The form of the Bayesian estimator that chooses the best set of estimates $\langle\alpha\rangle$ as that for which $p\{\boldsymbol{\alpha} \mid \mathbf{y}\}$ attains its maximum values becomes then equivalent to a Markov estimator as defined above [e.g., Schweppe, 1973, pp. 394-395]. Likewise the ML estimator becomes equivalent to a Markov estimator [e.g., Åström, 1980].

\subsection{Algorithms for the Implementation of Batch Estimation Schemes}

It is rarely possible to implement exactly the functional relationships of (25). It is at best possible to treat $\mathbf{y}$ and $\alpha$ as discrete random variables, not to mention the considerable debate that hinges upon the question of whence derive the necessary prior probability density functions (in this latter respect, Tiwari's [1979] discussion of the maximum-entropy principle for the specification of the prior functions probably reflects the majority of current thinking). In the following review of the literature there is only one example of an approximate implementation of (25) [Moore and Jones, 1978] and no example of an ML estimator applied without the assumption of Gaussian probability density functions.

Nearly all the case study problems amount therefore to the implementation of algorithms for locating the minimum of a loss function such as, in general, $J^{*}$ of (24). In general too, unless $\mathbf{e}\left\{\mathbf{x}_{a}\right\}$ is a linear function of $\mathbf{x}_{a}$, such that

$$
\partial J^{*}\left\{\mathbf{x}_{a}\right\} / \partial \mathbf{x}_{a}=\mathbf{0}
$$

yields a set of algebraic equations linear in $\mathbf{x}_{a}$, the estimation problem is said to be nonlinear. The important role played by the discrete-time input/output models of the form of (20), say, where the output response variables are linear functions of the model parameters, should become now much more apparent; they yield solutions to (26) that are linear in $\mathbf{x}_{a}$ (or simply $\alpha$ ) and hence easy mechanizations of the solution to the estimation problem.

To this general nonlinear estimation problem there are three aspects: choice of the definition of the error $\mathbf{e}$, the scheme for locating the minimum of $J^{*}$, and computation of estimates of the a posteriori parameter estimation error covariance matrix.

Clearly, it is the scheme of locating the minimum of $J^{*}$ that is usually regarded as being central to the practical numerical aspects of solving the estimation problem, and both Schweppe [1973] and Eykhoff [1974] discuss the details of several such optimum-seeking methods. The subject of optimization is, of course, an entire discipline in its own right, and we shall here merely outline certain features of these methods that have special relevance to other topics of concern to this review.

5.2.1. Specification of the form of error. As already noted, our discussion is restricted to the case of a single constituent error term (e), i.e., that between estimated and observed output response variables, defined either as the deterministic model response error of (23) or as the one-step-ahead prediction errors (or innovations process errors) introduced in section 4 as

$$
\mathbf{e}\left(t_{k}\right)=\mathbf{y}\left(t_{k}\right)-\left\langle\mathbf{y}\left(t_{k} \mid t_{k-1}\right)\right\rangle=\mathbf{v}\left(t_{k} \mid t_{k-1}\right)
$$

where

$$
\left\langle\mathbf{y}\left(t_{k} \mid t_{k-1}\right)\right\rangle=\mathbf{h}\left\{\left\langle\mathbf{x}\left(t_{k} \mid t_{k-1}\right)\right\rangle,\left\langle\boldsymbol{\alpha}\left(t_{k-1} \mid t_{k-1}\right)\right\rangle\right\}
$$

for comparison with (13).
The choice of $\mathbf{e}\left(t_{K}\right)$ as a deterministic model response error leads to a much simpler computational effort, both for the errors themselves and, should it be necessary, for estimating $W$ as the covariance matrix of $\mathbf{e}\left(t_{k}\right)$ [e.g., van Straten, 1983]. Fewer assumptions are needed than for the choice of $\mathbf{e}\left(t_{k}\right)=$ $v\left(t_{k} \mid t_{k-1}\right)$ in (28), which in fact requires the use of a Kalman filtering algorithm in order to compute $v\left(t_{k} \mid t_{k-1}\right)$ and its covariance matrix and thus implies as prerequisites all the panoply of assumptions and limitations listed earlier in section 4 (see also Åström [1980]).

5.2.2. Locating the minimum of the loss function. The implications of the choice of error definition are best clarified by now considering in more detail the scheme for locating the minimum of $J^{*}$. Among several possible forms of algorithm for a batch estimation scheme, the following gradient algorithm is widely used (see also Eykhoff [1974]):

$$
\left.\left\langle\boldsymbol{\alpha}^{i+1}\right\rangle=\left\langle\boldsymbol{\alpha}^{i}\right\rangle-\psi^{i}\left[\partial J^{*} / \partial \boldsymbol{\alpha}\right]_{\boldsymbol{\alpha}=\left\langle\boldsymbol{\alpha}^{i}\right.}\right\rangle
$$

where, as in Figure $4 a,\left\langle\boldsymbol{a}^{i}\right\rangle$ is the constant vector of parameter estimates for the $i$ th iteration through the data from $t_{0} \rightarrow$ $t_{N} ;\left[\partial J^{*} / \partial \alpha\right]_{\alpha=\langle\alpha i\rangle}$ is a vector of gradients for the loss function hypersurface with respect to the parameters $\alpha$, and evaluated at $\alpha=\left\langle\alpha^{i}\right\rangle$; and $\psi^{i}$ is a gain matrix (to be determined in a variety of ways). For simplicity it has been assumed that the estimation problem is one of parameter estimation alone. If the prediction error form $\mathbf{e}\left(t_{k}\right)=\mathbf{v}\left(t_{k} \mid t_{k-1}\right)$ is chosen, $\boldsymbol{\alpha}=\left\langle\boldsymbol{\alpha}^{i}\right\rangle$ is substituted into the Kalman filtering algorithms, which are then used to compute $\left\langle\mathbf{x}\left(t_{k} \mid t_{k-1}\right)\right\rangle,\left\langle\mathbf{y}\left(t_{k} \mid t_{k-1}\right)\right\rangle$, and hence $\mathbf{v}\left(t_{k} \mid t_{k-1}\right)$ in a recursive fashion for $t_{0} \leq t_{k} \leq t_{N}$. The filter in this instance is being used solely for the purposes of state estimation, and this use is subordinate to the evaluation of $J^{*}$ at the end of each iteration through the block of data. It is via the minimization of $J^{*}$ according to (29) that parameter estimation takes place, not directly through the filter.

There are two matters arising from this discussion that may cause confusion. First, there is the method of quasilinearization [Bellman and Kalaba, 1965], which has both attracted considerable interest in the analysis of water quality/ecological systems [Bellman et al., 1966; Lee and Hwang, 1971; Stehfest, 1977; Roberts and DiCesare, 1982] and has also (mistakenly) attracted the interpretation of being a fifth type of estimator uniquely different from the four types already identified. The basis of the method is to linearize the nonlinear system equations, such as those of our class II model (equation (6)) so that $\mathbf{e}\left\{\mathbf{x}_{a}\right\}$ becomes a linear function of $\mathbf{x}_{a}$. Then, as stated earlier, (26) leads to a set of $(n+p)$ linear algebraic equations in the $(n+p)$ unknowns, i.e., the elements of the augmented state-parameter vector $\mathbf{x}_{a}$. Here $n$ is the order of the state vector and $p$ the order of the parameter vector. Because therefore quasi-linearization is one particular (iterative) numerical method for locating the minimum of $J^{*}$, it is not, despite the impression frequently given, yet another type of estimator. A lucid, concise, and instructive discussion of the method can be found in the work by Detchmendy and Sridhar [1965].

Second, there is the question of how the recursive estimation algorithms of section 4 can be related to the notion of minimizing a squared-error loss function such as $J^{*}$. In the simplest of cases the recursive (linear) least squares algorithm can be seen as a (recursive) formulation for the changes to the solution of (26), i.e., the estimates $\left\langle\mathbf{x}_{a}\right\rangle$ that would be made if the number of observation sets available for analysis were to change from, say, $(k-1)$ to $k$. (Recall that the loss function $J^{*}$ 
in (24) is specified in terms of the number of observations available and that for convenient forms of $J^{*}$ the solutions from (26) can be specified in closed form.) In the most complex of cases, we may note that the Kalman filter (and hence the EKF) can be interpreted as a Bayesian-type estimator furnishing a posterior probability density function $p\left\{\boldsymbol{\alpha} \mid \mathbf{y}\left(t_{k}\right), \mathbf{y}\left(t_{k-1}\right)\right.$, $\left.\cdots, \mathbf{y}\left(t_{0}\right)\right\}$, after the receipt of the new observation $\mathbf{y}\left(t_{k}\right)$, as a function of the prior probability density function $p\left\{\boldsymbol{\alpha} \mid \mathbf{y}\left(t_{k-1}\right)\right.$, $\left.\mathbf{y}\left(t_{k-2}\right), \cdots, \mathbf{y}\left(t_{0}\right)\right\}$. For the assumption of Gaussian distributions the mean $\left\langle\mathbf{x}_{a}\right\rangle$ and variance-covariance matrix $P_{a}$ are sufficient to characterize these probability density functions.

5.2.3. The posterior parameter estimation error covariance matrix. Computation of the a posteriori uncertainties of the estimated model parameter values is a matter that has been widely ignored. The required mathematical derivation is unfortunately difficult to summarize, and what follows may therefore seem somewhat opaque. The essential point to bear in mind is that the concept of identifiability, first introduced in section 1 , can be related to the sensitivity coefficients of the model parameters (see also section 2). These coefficients appear explicitly in the quantification of the model parameter uncertainties after identification of the model; and it is this "residual model uncertainty" that links the problem of identifiability with the subsequent analysis of prediction error propagation (to be discussed in section 7).

Briefly, if the errors $\tilde{\alpha}$ in the parameter estimates are defined as

$$
\tilde{\alpha}=\alpha-\langle\alpha\rangle
$$

the objective is to derive an expression for the variancecovariance matrix of these errors, i.e., $P^{P}=E\left\{\tilde{\alpha} \tilde{\alpha}^{T}\right\}$, where $E\{\cdot\}$ is the expectation operator and where $P^{P}$ can be computed from a knowledge of the observations and, as necessary, the values of the parameter estimates themselves. Given the general nonlinear relationship between $J^{*}$ of (24) and the parameters, a single key assumption is necessary:

That at the minimum value of the loss function $J^{*}$, as located by the estimation algorithm, the corresponding (best) estimates of the parameters $(\langle\boldsymbol{\alpha}\rangle)$ are close the "true" values of the parameters $(\alpha)$, so that by linearization of the output response variables $(y)$ whereby $\mathbf{y}$ is made a linear function of the parameters, the errors e of (23) or (27) are likewise made approximately linearly dependent upon $\alpha$ (or $\tilde{\alpha}$ through (30)).

Clearly, the results of the analysis facilitated by this assumption are strictly applicable only to small estimation errors $\tilde{\alpha}$. The linearization is a first-order approximation and, as such, parallels a first-order error analysis in the context of prediction. Here the errors of identification are made a linear function of the parameter errors, so that the latter can be computed given the former. Later (in section 7) it will be apparent that the errors of prediction are assumed to be a linear function of the parameter errors, so that (there) given the latter, the former can be computed.

Let us denote the approximate form of the loss function that results from the above assumption by $\bar{J}^{*}$. Differentiation of $\bar{J}^{*}$ with respect to $\langle\boldsymbol{\alpha}\rangle$ and setting these derivatives to zero, in line with (26), yields now a set of algebraic equations linear in $\langle\boldsymbol{\alpha}\rangle$. From this set of equations an approximate expression for $\tilde{\alpha}$ follows straightforwardly. It contains a matrix of sensi. tivity coefficients $\left[\partial y_{i} / \partial \alpha_{j}\right]$ evaluated for $\boldsymbol{\alpha}=\langle\boldsymbol{\alpha}\rangle$ and is a function of the sources of uncertainty assumed to have been relevant to the estimation problem. From the expression for $\langle\boldsymbol{\alpha}\rangle$ a means of approximating the variance-covariance matrix of these parameter estimation errors can be formulated as follows

$$
\begin{aligned}
& P^{P}\left(t_{N}\right)=E\left\{\tilde{\boldsymbol{\alpha}} \tilde{\boldsymbol{\alpha}}^{T}\right\}=E\left\{\left[\sum_{k=1}^{N} C^{T}\langle\boldsymbol{\alpha}\rangle W^{-1} C\langle\boldsymbol{\alpha}\rangle\right]^{-1}\right. \\
& {\left[\sum_{k=1}^{N} C^{T}\langle\boldsymbol{\alpha}\rangle W^{-1}\left\langle\boldsymbol{\eta}^{*}\left(t_{k}\right)\right\rangle\right]\left[\sum_{k=1}^{N} C^{T}\langle\boldsymbol{\alpha}\rangle W^{-1}\left\langle\boldsymbol{\eta}^{*}\left(t_{k}\right)\right\rangle\right]^{T}} \\
& \left.\cdot\left[\sum_{k=1}^{N} C^{T}\langle\boldsymbol{\alpha}\rangle W^{-1} C\langle\boldsymbol{\alpha}\rangle\right]^{-1}\right\}
\end{aligned}
$$

where, more precisely, $C\langle\boldsymbol{\alpha}\rangle$ is the $(l \times p)$-dimensional matrix of sensitivity coefficients $\left[\partial y_{i}\left(t_{k}\right) / \partial \alpha_{j}\right]$ for the $i$ th output variable with respect to the $j$ th parameter at time $t_{k}$ and evaluated for $\boldsymbol{\alpha}=\langle\boldsymbol{\alpha}\rangle$ (the output vector being of the order of $l$ ). The error sequence $\left\langle\boldsymbol{\eta}^{*}\left(t_{k}\right)\right\rangle$ is, strictly speaking, an estimate of the mismatch between the observed and hypothetical error-free output variables. In practice, $\left\langle\boldsymbol{\eta}^{*}\left(t_{k}\right)\right\rangle$ can be suitably approximated as the error $\mathbf{e}\left(t_{k}\right)$ between observed $\mathbf{y}\left(t_{k}\right)$ and estimated output variables $\left\langle\mathbf{y}\left(t_{k} ;\langle\boldsymbol{\alpha}\rangle\right)\right\rangle$.

For special cases of the errors $\eta^{*}\left(t_{k}\right)$, namely, that they are white noise sequences, the expectation operator in (30) can be taken to apply merely as $E\left\{\boldsymbol{\eta}^{*}\left(t_{k}\right) \boldsymbol{\eta}^{* T}\left(t_{k}\right)\right\}$, and thus $P^{P}\left(t_{N}\right)$ can be seen to be directly related to the variance-covariance matrix of the errors of mismatch. For the case of a model that is linear in the parameters, (31) will obviously be much simplified. In a qualitative sense one can infer from this equation that the more sensitive the output response variables are to the parameters (the elements of $C$ are relatively large), the smaller are the associated estimation error variances. The parameters would be said to be "better estimated" (less uncertain) in this event, although the ability of the parameters to be "well identified" is dependent upon the temporal pattern of the sensitivity coefficients over the whole period of the field observations (as already indicated in the discussion of Figure 2 in section 2).

\subsection{Case Studies}

The earliest contributions to parameter estimation in water quality/ecological systems, as would be expected, dealt with the theoretical promise of particular algorithms for the minimization of an ordinary least squares loss function $(J)$. They addressed simple process models for prey-predator dynamics [Bellman et al., 1966] or for the steady state relationship between DO and BOD concentrations along a reach of river [Lee and Hwang, 1971; Koivo and Phillips, 1971]. Parker [1972], with a seven-state variable model representing nutrient-phytoplankton-zooplankton dynamics in Kootenay Lake, British Columbia, Canada, and Shastry et al. [1973], in a study of DO-BOD interaction in the Sacramento River, California, were among the first to tackle the analysis of in situ field data. In fact, Shastry et al. regarded theirs as a problem of discriminating among alternative model structures.

The overwhelming majority of subsequent case studies have used, as did Shastry et al. [1973], either a weighted least squares or Markov estimator for which the error term has been defined as a deterministic model response error according to (23). (The use of the term Markov estimator signifies here a maximum likelihood estimator with the customary Gaussian assumptions.) Many of these applications have focussed, with varying degrees of complexity (from one to 17 
state variables), on the development of models for phytoplankton dynamics in lake ecosystems [e.g., Di Cola et al., 1976; Lewis and Nir, 1978; Benson, 1979; Canada Centre for Inland Waters, 1979; Di Toro and van Straten, 1979; Jorgensen et al., 1981; van Straten, 1983; Mejer and Jorgensen, 1983]. Stehfest's [1977] analysis of a six-state variable model for the steady state distribution of degradable and nondegradable organic pollutants along a $450-\mathrm{km}$ stretch of the Rhine River, Federal Republic of Germany, is a notable exception to this general pattern (see also Rinaldi et al. [1979a]). Should such statistics be of interest, we may observe that Di Toro and van Straten [1979] and van Straten [1983] can claim to have addressed one of the largest scale problems of estimation. They have used a 12-state variable model with in all, 20 parameters to be estimated, in analyses of the International Field Year for the Great Lakes (IFYGL) 1972-1973 data and an aggregated 10year data set (1967-1976) for Lake Ontario.

The considerably more onerous estimation problem associated with the (one-step-ahead prediction) error definition of (27) has understandably been less extensively addressed. Part of the analysis of model structure identification for the study of DO-BOD-algae interaction in the River Cam [Beck, 1975, 1978] involved an ML (Markov) estimation problem using the algorithm of Aström [1980]. Results from an identical approach to the estimation of 33 parameters (including fivesystem noise $\boldsymbol{\xi}$ and measurement noise $\boldsymbol{\eta}$ covariance elements) in a six-state variable hydrothermal model for the inlet water temperature of an electricity-generating station have been reported by Schrader and Moore [1977] and Moore [1978]. Jolankai and Szöllösi-Nagy [1978] have formulated an algorithm for a similar estimation problem associated with a model of phosphorus-cycle dynamics in the Bay of Keszthely, Lake Balaton. However, they acknowledge the difficulties of implementing such an algorithm and have proposed, but not applied, alternative simpler batch and recursive estimation schemes that exploit ideas along the lines of a quasilinearization approach. McLaughlin [1978a, 1979] has also been a strong advocate of ML (Markov) estimators for criteria $\left(J^{*}\right)$ based on one-step-ahead prediction errors. His comparative account of the performance of the two forms of estimation schemes implied by the two different error specifications is especially instructive, though sadly never published in the open literature [McLaughlin, 1979].

Without doubt the most sophisticated type of estimator applied hitherto is an approximate version of the Bayesian estimator proposed by Moore and Jones [1978]. In their algorithm, which they call a coupled Bayesian/Kalman filter estimator, they have approximated the continuous ranges of the parameters $\alpha$ by a finite set of discrete values. For a model with $p$ parameters to be estimated, each of whose range of possible values is represented by $M$ discrete values, there are thus $M^{p}$ possible realizations of the model corresponding to all the possible combinations of the parameter values. The prior and posterior probability density functions $p\left\{\boldsymbol{\alpha} \mid \mathbf{y}\left(t_{\boldsymbol{k}-1}\right)\right\}$ and $p\left\{\alpha \mid \mathbf{y}\left(t_{k}\right)\right\}$ (before and after processing the observation $\left.\mathbf{y}\left(t_{k}\right)\right)$ are likewise represented by $M^{p}$ discrete probabilities. Such a formulation of the estimation problem would indeed be attractive if it were not for the very high computational cost of implementing the approach. $M^{p}$ Kalman filters are required to compute the errors $v\left(t_{k} \mid t_{k-1}\right)$, and their associated variance-covariance matrices for each of the $M^{p}$ possible realizations of the model. Knowledge of these Gaussian error dis- tributions is necessary for the computation of $p\left\{\mathbf{y}\left(t_{k}\right) \mid \boldsymbol{\alpha}\right\}$ appearing in the right-hand side expression of the appropriate form of Bayes' rule as stated in (25). Intuitively, if the errors from a particular model are high, it is improbable that the actual observation $\mathbf{y}\left(t_{k}\right)$ has been generated from the particular combination of parameter values that constitute that model. It is apparent too that a set of recursive estimates can be computed from the sequence of "most probable" parameter value combinations at each instant $t_{k}$ over the period $t_{0} \rightarrow t_{N}$. Some of Moore and Jones's [1978] results for the River Cam data of Beck and Young [1976] are shown in Figure 12. They are indicative of the problems of parameter identifiability discussed in section 2 . The flat marginal distribution of a posteriori probabilities (at $t_{N}$ ) for the reaeration rate constant $\alpha_{2}$ in Figure $12 c$ is little different from the prior probabilities (at $t_{0}$ ) of $1 / 9$ for each of the nine possible discrete values for this parameter. The corresponding posterior estimation error variance in $P^{P}\left(t_{N}\right)$ would be relatively high for $\alpha_{2}$. The conclusion is that there is little information in the field observations with which to identify a value for the reaeration rate constant. And this is not surprising, for in the Cam study and in the Bedford Ouse the effects of stream reaeration mechanisms on the observed DO variations are negligibly small in comparison with the dominant effects of algal photosynthesis and respiration [Beck, 1983].

\subsection{Commentary: The Problem of Identifiability}

This by no means exhausts the coverage of all the case studies relevant to parameter estimation. However, the already evident problems of parameter identifiability are so commonplace, and have so frequently been responsible for the disappointing performance of the various estimation algorithms, that they must now be given special consideration.

To summarize, there are many difficulties in working with in situ data from environmental systems: there are too few data and/or they are highly uncertain, the parameter space has too high a dimension and/or the optimal estimates of the parameter assume "unrealistic" values, there is a lack of convegence to optima on the error-loss function hypersurface because of its flatness and/or unless reasonably good prior parameter estimates can be specified, and the necessary prior knowledge of the various error statistics is itself uncertain.

The nub of the problem of identifiability is (as we have said in the introduction to this review) that what we would like to know about the internal description of the system $\{\mathbf{x}, \alpha\}$ is of a substantially higher order than what can be observed about the external description of the system $\{\mathbf{u}, \mathbf{y}\}$.

Three groups of factors affect the shape of the estimation loss-function surface: (1) the field observations, (2) the model structure (and its parameters), and (3) the type of estimator, i.e., the prior assumptions about the statistics of the sources of uncertainty affecting the estimation problem. We shall categorize the various responses to the problem of identifiability according to these three points.

5.4.1. Manipulating the data. A predictable response to the difficulties of identifiability is to transform the given data set before any analysis-by interpolation ("increasing" the number of data) and/or by smoothing ("removing"part of the random uncertainty). Mejer and Jorgensen's [1983] use of cubic-splines fitting is a typical example of this response. In fact transformation of the sampled data by interpolation 

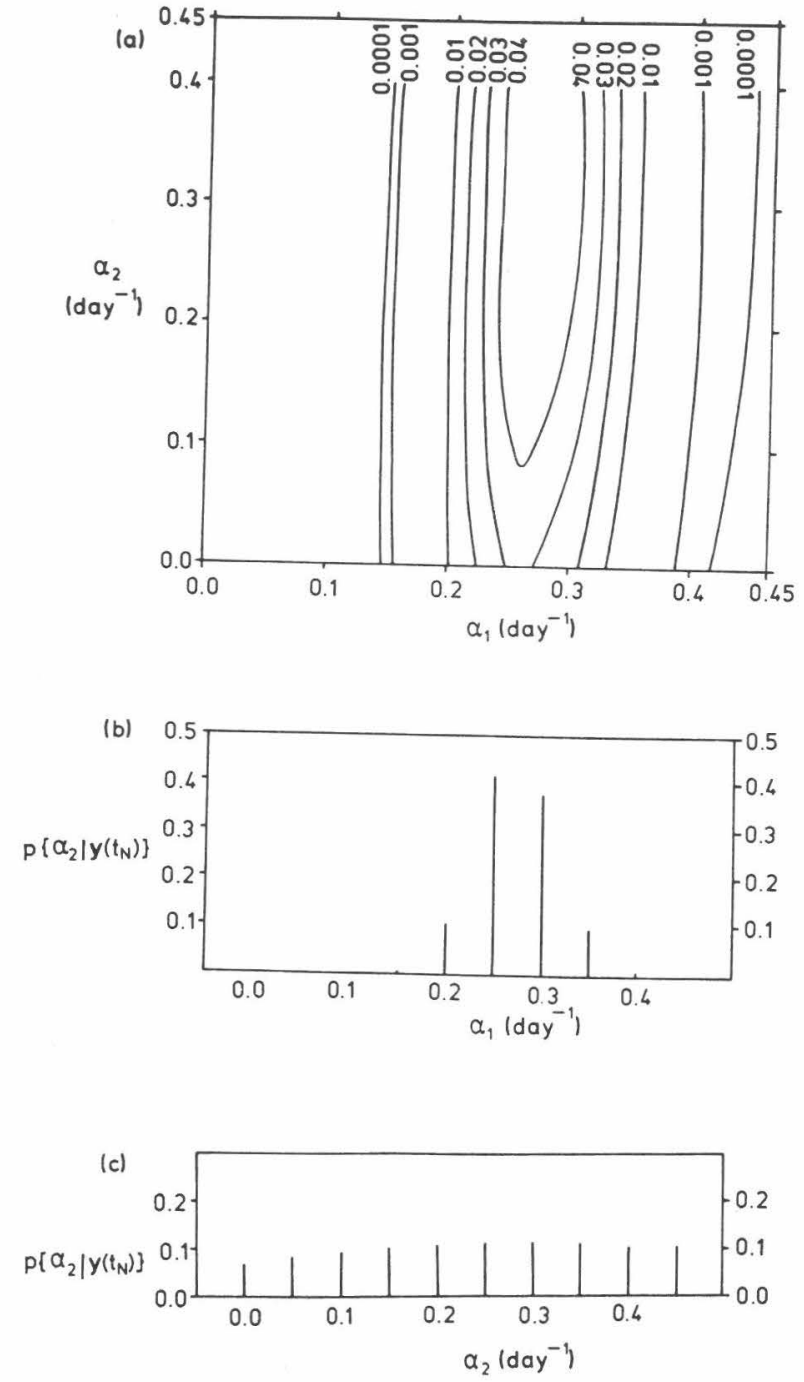

Fig. 12. Parameter estimation in a case study of the River Cam [after Moore and Jones, 1978]. (a) A posteriori joint probability density function at $t_{N}(N=80$ sampled observations) for the BOD decay rate constant $\alpha_{1}$ and reaeration rate constant $\alpha_{2}$. (b) A posteriori marginal probability density function $p\left\{\alpha_{1} \mid \mathbf{y}\left(t_{N}\right)\right\}$ for the BOD decay rate constant $\alpha_{1}$. (c) A posteriori marginal probability density function $p\left\{\alpha_{2} \mid \mathbf{y}\left(t_{N}\right)\right\}$ for the reaeration rate constant $\alpha_{2}$.

and/or smoothing (filtering) raises a more general issue, since arguably it transforms the estimation problem in a fundamental way (see also Young and Jakeman [1980] and Vajda et al. [1985]).

Consider once again the ordinary differential-equation representation of the class II model structure, i.e.,

$$
\begin{gathered}
d \mathbf{x}(t) / d t=\mathbf{f}\{\mathbf{x}, \mathbf{u}, \boldsymbol{\alpha} ; t\}+\xi(t) \\
\mathbf{y}\left(t_{k}\right)=\mathbf{h}\left\{\mathbf{x}, \boldsymbol{\alpha} ; t_{k}\right\}+\boldsymbol{\eta}\left(t_{k}\right)
\end{gathered}
$$

Hitherto it has been assumed that in order to solve the problem of deriving values for $\alpha$ the differential equation (32a) must first be solved and then some function of the errors e $\left(t_{k}\right)$ minimized, taking into account sampled observations given at times $t_{k}, k=1, \cdots, N$. If we permit a manipulation of the data $\left\{\mathbf{u}\left(t_{k}\right), \mathbf{y}\left(t_{k}\right)\right\}$ by interpolation and smoothing, it is possible to formulate a modified estimation problem constructed around an equation of the form

$$
\left\langle d \mathbf{x}\left(t_{j}\right) / d t\right\rangle=\mathbf{f}\left\{\langle\mathbf{x}\rangle, \mathbf{u}, \boldsymbol{\alpha} ; t_{j}\right\}+\varepsilon\left(t_{j}\right)
$$

Here $\left\langle d \mathbf{x}\left(t_{j}\right) / d t\right\rangle$ is an estimate of the derivative of the state vector evaluated at sampling instants $t_{j}, j=1, \cdots, M$, and $\varepsilon$ is a lumped-error term accounting for the sources of uncertainty originally associated with $\xi$ and $\eta$ in (32) but transformed through whatever procedure is used to obtain $\left\langle d \mathbf{x}\left(t_{j}\right) / d t\right\rangle$ and $\left\langle\mathbf{x}\left(t_{j}\right)\right\rangle$.

The key is that the estimation problem now refers to the evaluation of $\alpha$, being the only unknown in the algebraic (or regressionlike) relationship of (33), given "observations" at times $t_{j}$ where, by interpolation, the number of samples $(M)$ can be made substantially larger than for the original problem (i.e., $N$ ). The algebraic form of (33) is structurally equivalent to that of the input/output class III model (equation (3)), and as such, the transformed problem has access to all the corresponding types of estimation algorithm (including the IV estimator) that experience shows have performed well on the difficult problems of environmental time-series analysis.

The price to be paid for this transformation is the need to generate estimates of the state $(\langle\mathbf{x}\rangle)$ and, more important, estimates of the state derivative $(\langle d \mathbf{x} / d t\rangle)$. The simplest such substitution, for the case where all the states are linearly observed as the outputs (y) (as is usually assumed) is to put $\langle\mathbf{x}\rangle=\mathbf{y}$ in (33) and to difference the data (y) in order to obtain approximate estimates of $\mathrm{d} \mathbf{x} / d t$. If further it is assumed that $\varepsilon=0$ in (33), then estimates of $\alpha$ can be derived as the solutions to a set of deterministic nonlinear algrebraic equations, a form of solution well known as the solution to the inverse problem of groundwater models (McLaughlin [1978a]; see also Jorgensen et al. [1981] for the case of lake eutrophication models). The differencing of error-corrupted data is never a good idea, however, since in general it amplifies the effects of the errors. The use of cubic splines should yield more stable approximations of the derivatives (together with their interpolated values), but they are essentially the same form of substitution given that they require $\langle\mathbf{x}\rangle=\mathbf{y}$ at the sampling instants of the original data, i.e., at $t_{k}$ [Vajda et al., 1985]. Other means of deriving stable estimates of $\langle\mathbf{x}\rangle$ and $\langle\mathrm{d} \mathbf{x} / d t\rangle$ naturally suggest themselves, of which an instrumental variable form is one (as discussed by Young and Jakeman [1980] and Vajda et al. [1985]) and an estimate from a Kalman filter is another.

Above all, the transformed estimation problem for the relationships of (33) opens up new horizons for the estimation of parameters in ordinary differential-equation models by methods developed originally for discrete-time, difference-equation representations. The one qualification of this potential is that it must first be possible to obtain good approximations of the values of the state derivatives.

5.4.2. Manipulating the parameter space. Imposing a restriction on the number of model parameters to be estimated, i.e., reducing the dimension of the parameter space, is complementary to increasing the number of data. The question then is, on what basis should one distinguish between those parameters that are to be estimated and those that are to be either assigned values a priori (from a search of the literature) or removed altogether from the given model structure?

In fact the question is tantamount to asking how one identifies the model structure, except that here it is being approached from a direction opposite to that already discussed 
in section 4. The problem is one of establishing which constituent model hypotheses are, in effect, either redundant or not falsifiable (surplus content). It follows from the discussion of identifiability at the end of section 2 that some form of sensitivity analysis would be a suitable vehicle for determining the insensitive or redundant parameters, and there are several case studies that illustrate this approach [Whitehead and Hornberger, 1984; Jaffe and Ferrara, 1984; Hornberger and Cosby, $1985 a, b ;$ Gentil and Perrier, 1985].

There are other strategies, and indeed other questions, of a more subtle and philosophical nature about the justification for restricting the dimension of the parameter space. Besides eliminating, or "fixing" those parameters to which the outputs of the model (y) are insensitive, it has been argued that the most "uncertain" parameters are equally so candidates for elimination [van Straten, 1983]. In other words, the estimation problem is first solved for the model given all the parameters as unknown constants; the error variance-covariance matrix of the parameter estimates is then computed according to (31), and on this basis a revised estimation problem is devised with the most uncertain parameters having been eliminated. However, this is in effect the same strategy as elimination of the least sensitive parameters (especially as (31) explicitly incorporates the sensitivity coefficients), except that the procedure is carried out by reference to the experimental observations (as in the works by Whitehead and Hornberger [1984] and Hornberger and Cosby $[1985 a, b])$ and not solely on the basis of the intrinsic properties of the given model structure [Jaffe and Ferrara, 1984; Gentil and Perrier, 1985].

The fixing of certain parameter values is equivalent to assuming a priori that these parts of the model structure are known with certainty, and such an assumption must necessarily distort the posterior estimate of the variance-covariance structure of the parameter estimation errors, as van Straten [1983] has illustrated in his analysis of a model for Lake Ontario. It is clear that this is a rather contentious issue, not only from the point of view of parameter estimation, but also because it brings into question the role of surplus content in the context of prediction and prediction error propagation. As yet these questions have barely been addressed, let alone resolved, and we shall therefore postpone further discussion of them until section 8 , where they are treated in an agenda of problems for the future.

One point is apparent, nevertheless, and it is that to fix the most uncertain parameters seems a logical contradiction. It implies perfect knowledge of the least certain parameters. To allow such parameters to assume random values (within a prespecified range) is preferable and is an approach that has been discussed extensively by Hornberger and Cosby [1985b] and Hornberger et al. [1985] in a case study of surface water acidification.

Probably the most exhaustive treatment of parameter-space delimitation thus far is presented in a recent paper by van Straten [1985] with reference to a case study of eutrophication in Lake Balaton, Hungary. He has introduced quite novel ways of exploiting the observed dynamic characteristics of the system (for instance, peak response, period average, and steady state responses) in order to impose constraints on the feasible regions of those parameters to be estimated. But what precisely determines a priori a "feasible" range of parameter values is quite another philosophical issue. Sorooshian et al. [1983], in an analysis of the identifiability of hydrological models, have observed that should unrealistic values be estimated for the parameters, their preference is not to impose such constraints on the estimator, but to challenge the adequacy of the estimation criterion, i.e., the type of estimator, or the form of the prior statistical assumptions. The position of the present reveiw would be different still, seeing it as evidence of the failure of a constituent model hypothesis, and thus part of the natural duality of failure and inference that is the essential character of model structure identification.

5.4.3. Manipulating the form of estimator. There is substantial evidence in the parallel literature on hydrological modeling of these same problems of parameter identifiability [Johnston and Pilgrim, 1976; Pickup, 1977; Sorooshian et al., 1983], and much the same conclusions have been drawn, namely, that the key problems may ultimately be ones of model structure identification and the temporal variability of parameters [Sorooshian et al., 1983] (see also section 4 of this paper).

In particular, considerable attention has been given by Sorooshian and Gupta [1983] and Gupta and Sorooshian [1983] to examination of the loss-function surface. Few, if any, of the studies in water quality modeling have been as exhaustive an the analysis of these authors, although it is clearly well known that the loss-function surface can degenerate to a very awkward, almost flat shape. For example, in a study of a phosphorus- and carbon-cycle model of Lake Ontario, Halfon [1979] has noted that an order-of-magnitude variation in some parameter values might be associated with a change of no more than $6 \%$ in the loss function about its minimum value. So changing the form of the estimator, i.e., changing the assumptions about the properties of the sources of uncertainty, in response to a lack of model identifiability is a third course of action, and one advocated by Sorooshian et al. [1983]. In fact they discuss the autocorrelation and heteroscedastic properties of the output response observation errors, and their results with the revised estimators were favorable.

The results of other studies have not been so successful [Hornberger et al. 1985], and it remains an open question as to how suitable this type of transformation would be in identifying models of water quality. For one thing it is not obvious that the relative lack of water quality data would support the more sophisticated statistical assumptions.

This is not to suggest, however, that the importance of making good assumptions about error and noise statistics (as required for the Markov, ML, and Bayesian estimators) has not been acknowledged in estimating the parameters of water quality models. It is obviously apparent in the preceding discussion of section 4 in respect of recursive estimation algorithms, though not in as refined a fashion and not in quite the same context as Sorooshian's work. Moore [1978] too, for instance, has given some discussion of adaptive (Kalman) filtering techniques, "adaptive" in the sense that the unknown elements of the variance-covariance matrices for the different sources of uncertainty are treated as parameters (themselves to be estimated from analysis of the field data). But again, adaptive filtering is really quite a sophisticated notion, and its robustness under the difficult conditions of interest here must be questionable.

Last, an alternative position on the problem of identifiability is to accept the multiple optima as given and, instead of attempting to manipulate the estimator, to develop procedures for identifying from the multiple optimal solutions some 
"uniquely best" combination of parameter estimates. Such procedures have been discussed at length by Lewis and Nir [1978] and Paldor et al. [1978], although they recognize the objections that might be raised, and have been raised by Fedra [1983], over the arguably illusory assumption of uniqueness (see also section 3).

\subsection{Prudent Transformations of Ill-Posed Problems}

Our conclusion must be the same as that to the discussion of model structure identification in section 4 . If the most natural and straightforward formulation of the estimation problem continues to defy useful solution, then it is prudent to change the formulation of the problem.

There are two pairs of case studies that can be reviewed in this spirit. The first such pair refers to the work of van Straten and Herodek [1982], on a problem of estimating algal growth parameters from primary production profile observations in Lake Balaton, and to the analysis of Rinaldi et al. [1979 b] of steady state DO-BOD interaction in the Bormida River, Italy. Both studies transform and simplify the natural statement of their respective estimation problems. This is followed by exploiting the partly linear properties of the minimization of a weighted squared-error loss function $\left(J^{*}\right.$ in (24)), which substantially reduces the subsequent computational effort of an iterative scheme for locating the minimum of $J^{*}$. The final sets of parameter estimates obtained from several independent sets of observations are then examined for the identification of possible functional relationships between the parameters and (observations of) the other independent variables (see also Cosby [1984]). This last step is clearly an expression of the idea of model structure identification as associated with the discussion of (14) in section 4.

A second, but rather different, pair of case studies can be found in Halfon's [1976] analysis of the dynamics of selenium in an aquatic microcosm and Roberts and DiCesare's [1982] work with a simple model for nutrient dynamics in Lake George, New York. The common theme of these studies is the transformation of the basic system description of a class II model, i.e., (32), into the discrete-time form of the multipleinput/multiple-output class III model of (3). The importance of the concept of linearity in the parameters, irrespective of whether the system dynamics are a nonlinear function of the states, inputs, or outputs, is particularly well demonstrated by Roberts and DiCesare's analysis. Most probably it is this linearity that makes their estimation problem so much better posed and the results of their analysis more penetrating.

5.5.1. Distributed-parameter (class I) models. The archetypal problem of identifiability, as we have already indicated in the introduction (section 1), is that of estimating the parameters in the distributed-parameter (partial-differential equation) representation of a class I model. And since all the systems considered in water quality modeling are intrinsically distributed, all the case studies discussed thus far have assumed implicitly the prior transformation of the natural formulation of the problem (identification of the distributedparameter model) into a "more tractable" lumped-parameter approximation.

The question remains therefore whether this transformation is necessary and/or prudent, and the answer to this question must largely be positive (on both accounts). Distributedparameter systems are by definition of infinite dimension (in terms of the orders of their state $\mathbf{x}$ and parameter $\boldsymbol{\theta}$ vectors), and some form of lumping approximation is inevitable, usually over small volumes for the state vector and over much larger volumes for the parameter vector. Most of the results have been restricted to the essentially hydraulic problems of the advection and dispersion of conservative pollutants in rivers [Yih and Davidson, 1975; Thé, 1978; van Straten et al., 1985; Budgell, 1982] and in groundwater systems, including the upper unsaturated zones [McLaughlin, 1978a, b, 1979, 1985; Wheater et al., 1986; Neuman and Carrera, 1985]. The exceptions to this rule are the results of Koivo and Phillips [1976] and Koivo and Koivo [1978] for the theoretical development of recursive least squares estimators for a first-order partial-differential equation representation of stream DO-BOD interaction. It would not be fair to observe that the problems of identifiability have dominated these studies, for there have barely been any exhaustive analyses of even reasonably extensive field data sets. What meager evidence there is derives from a case study of conductivity time series from the upstream and downstream boundaries of an 84-km section of the River Rhine in Holland [Thé, 1978; Bagchi et al., 1980; van Straten et al., 1985] and focuses on the poor identifiability of the dispersion coefficient; a consequence partly of insufficient high-frequency input excitation of the system's dynamics, and partly of the nonstationarity of the mean stream velocity. However desirable it may be to maintain that faithful representation of the distributed nature of the system should be compromised as little as possible, the fact is that there are few practical case study results that would dissuade one from invoking a priori a lumping transformation of the estimation problem.

Rather the real question is therefore whether class I model representations can be useful as vehicles for the rigorous interpretation of field data. And if they cannot, it they must be viewed merely as archives of hypotheses, can these hypotheses be translated more effectively into the more easily identifiable class II and III model structures (other than via finite-element and finite-difference approximations)? Indeed, can the results of identifying the latter be translated back into the context of the partial-differential equation model? These are, in the first instance, extremely difficult questions to answer on a technical basis. For example, the overall problem could be transformed into the dual (but not independent) subproblems of identifying purely temporal or purely spatial variability of behavior, with estimates of the spatial and temporal derivatives, respectively, being accommodated in much the same manner as discussed above for (33) in section 5.4 .1 (see also section 8 and Beck [1985b]). But perhaps of greater and more fundamental difficulty are the philosophical issues raised by these questions. For it may be that here we are witnessing a small dislocation of paradigms (in the sense of Kuhn [1970]) in the representation of the dispersive processes affecting the distribution of a solute in a flowing medium. It is now amply established that a class III model structure is able to match accurately, with generality, and with parsimony of parameters the transport of tracer substances in streams and channels under a wide variety of conditions ГYoung, 1983; Beer and Young, 1983; Young and Wallis, 1986]. The difficult philosophical issue is that the understanding gained from interpretation of the field data in the terms of such (class III) models is fundamentally not reconcilable with the interpretation of the customary class I model formalism. The two are based on different premises, and it may not therefore be meaningful to seek 
the failure of the one set of hypotheses by their translation into the other form of representation.

5.5.2. In conclusion: $A$ "forensic" science. What should have been a straightforward problem with a straightforward solution has in the event turned out to be remarkably intractable. Indeed, the skeptic faced with such a long list of difficulties as those enumerated in section 5.4 above might well argue that any form of parameter estimation is a pointless exercise. He would be quite wrong, and emphatically so. For at this point in the review it must hardly be necessary to reiterate the basic philosophical position of seeing the failure of an approach as the stimulus to insight and progress. This has been true in the development of methods for the generation of preliminary hypotheses (section 3), and it promises also to be the case in generating novel algorithms for model structure identification (section 4). The only difference between these and the present discussion is that perhaps perversely it is all the more difficult to acknowlege the failure of a body of methods (i.e., constrained optimization methods) that are ostensibly so readily applicable to the problem at hand. But like the preceding topics, the most fruitful development should err on the side of simplicity and independence of insight, not (as with the encroaching complication of the HSY approach, or the inordinate superstructure built on to the use of recursive estimation algorithms) in the ad hoc tinkering with and sophistication of existing methods.

If today the subject of parameter estimation were to be presented with an outstandingly best algorithm for constrained optimization, would all its problems be solved? It is doubtful, although there are those who would claim to have such a global optimization procedure [Pinter et al., 1987]. This review has indicated that the application of standard procedures for the minimization of a fitting-error function is fraught with difficulties. Moreover, it has been argued elsewhere that the expense of computational time should be regarded as a progressively receding resource constraint on numerical analysis. Why then should one use efficient algorithms for locating the minimum of the fitting-error function when inexpensive computing resources can be mobilized in order to evaluate the entire hypersurface of this function over given ranges of the parameter values? The thinking behind the negative response that this question prompts is already apparent in the studies reported by Halfon [1979], Fedra [1983], Hornberger and Cosby [1985b], and Hornberger et al. [1985]. Ironically too, it is this approach of complete function evaluation that is used for the very purpose of diagnosing the failure of an optimization procedure to perform adequately [e.g., Sorooshian and Gupta, 1983]. Presumably the difficulty of analysis might shift its focus away from locating the optima to interpretation of their meaning, and it is unlikely therefore that further sophistication of the methods of optimization will be especially rewarding. To summarize, either the problem must be changed to allow existing methods to perform better (as we discuss below), or some quite radical change of method will be required in order to open up novel ways of approaching the problem.

The difficulties of parameter estimation are not primarily ones of inadequate method, but of a latent ill posedness of the problem arising from a lack of identifiability. It would thus be inappropriate to conclude by discriminating among good and bad techniques. The issue is one of developing preferred approaches to problem solving. The essential problem is that of identifiability, and the essence of identifiability is ambiguity in the interpretation of past observed behavior. The case in favor of a variety of perspective and approach, first put forward in section 4 on the discussion of model structure identification, is now even more compelling. The problems of estimation are unlikely to be solved at a single attempt from one direction. Formulating the model structure (without identifying it), defining a least squares loss function, and putting the problem into a constrained optimization package does not automatically produce a useful solution. It may produce part of the solution, but a greater ingenuity is called for. The problem must be approached from several different angles, and the diagnostic evidence from each partial, albeit ambiguous, solution then assembled to make a cogent argument for choosing one solution (among several) to the whole. The interpretation of field data should be, as it were, a kind of forensic science. And if there were to be a single most important guiding principle of this approach-of continual transformation and recasting of the estimation problem - it would be defined as follows.

The judicious use of assumptions designed to produce a model specified in terms that approximate the algebraic or discrete-time difference form of a class III representation that is linear in the parameters to be estimated.

In the end, however, it may be that the ambiguity of interpreting past behavior, though undesirable, is not fatal to the purpose of predicting the future, and this would certainly challenge the accepted attitudes to the problem of identifiability set out above.

\section{Checks AND Balances}

There is no automatic or entirely objective routine for the development of a model, not even in the restricted area of model-order estimation for the input/output class III representations [Söderström, 1977]. The procedure is natually cyclical, iterative, and "open ended" (see also Cale et al. [1983a], Loehle [1983], and Reckhow and Chapra [1983a]. As described here, it alternates between the speculative generation of hypotheses (section 3), exposure of their failure, then further speculation (in section 4, model structure identification), and so on. The procedure is incremental, whether one begins with an overly simple or an overly complex model structure. To paraphrase the discussion of section 4 , additional or more complex hypotheses are only incorporated into the simple prior model structure when they are demonstrably necessary. And to paraphrase what has been said in section 5, having included a priori in the model all the hypotheses of conceivable relevance, the alternative approach is then to seek to eliminate those hypotheses that are clearly inadequate or redundant (an approach likely to be heavily circumscribed by the difficulties of identifiability).

The question that we must now answer is at what point should the analysis terminate? How can it be established that we have developed a good, useful, valid, credible, realistic, or desirable model? The answer depends to a large extent on the purpose for which the model has been developed, and equally so on a large measure of common sense; it is also subjective [Young, 1983]. The question can be made retrospective: does the model adequately match observed past behavior? Or it can be made prospective: is the risk acceptable that the model can be used to guide decisions that will influence future behavior? A satisfactory answer to the former, though perhaps 
easier to obtain, clearly does not imply a satisfactory answer to the latter. Neither is it apparent that one could examine the objectives of decision making, specify an acceptable tolerance on the requisite prediction accuracy, and hence specify as a terminating point for identification some acceptable level of identified model uncertainty.

So we are obliged to accept some less crisply defined distrinction between an acceptable and an unacceptable model, some less precise notion of the end point of system identification. And we shall consider in this section the simpler, strictly "retrospective" aspects of this problem.

The purpose of the whole of the review thus far has been the use of models for the development of scientific theories about the behavior of complex environmental systems. Given this purpose, a pragmatic prescription for the end point of the analysis would require the following.

1. The parameters of the identified model structure should be demonstrably invariant with time (from section 4).

2. The estimation error variances and covariances of these parameters should be low, as evidence of good identifiability (from section 5).

3. The residual errors of mismatch between the performance of the model and observed behavior should be small in magnitude and not attributable to any causal mechanisms of a significantly nonrandom character.

Items 1 and 2 have already been discussed earlier. In this section the primary concerns will be analysis of the residual errors of mismatch and, because it is frequently based on such an analysis, model discrimination.

\subsection{Analysis of the Residual Errors of Mismatch}

In line with (23) and (27) in section 5 the residual errors of mismatch can be generated as either the deterministic model response errors or the one-step-ahead prediction errors, respectively (the posterior parameter estimates being substituted for the parameter values). The former are usually a more stringent test of model adequacy; the latter can only be generated via some form of filtering or recursive estimation algorithm.

Analysis of the statistical properties of the residual errors can take several forms, from more to less aggregated. Thomann [1982], for example, has proposed a number of "verification measures" covering regression coefficients (derived from regressing the observed response $\langle\mathbf{y}\rangle$ on the estimated output y) and aggregate statistics such as a relative error and a root-mean-square error (see also Thomann and Winfield [1976] and Thomann et al. [1979]). These are aggregated in the sense that such statistics refer neither to the temporal sequence of the errors nor to the adequacy of the constituent model hypotheses (a pitfall that Thomann also notes when he cautions that "... model error statistics may be excellent but the wrong mechanisms are included in the model" [Thomann, 1982]). Reckhow and Chapra [1983a] have suggested a somewhat broader range of "confirmation measures" and have given particular attention to the role of temporal correlation among the residual errors in distorting the evaluation of some of these aggregate statistics.

The analysis can, of course, be more detailed, especially when adequate time-series data are available and when explicit assumptions have been made about the statistical properties of the various sources of uncertainty assumed to influence the identification process (as discussed in section 4). For example, if we suppose that the one-step-ahead prediction errors (or innovations process errors) $v\left(t_{k} \mid t_{k-1}\right)$ can be gener- ated from a filtering algorithm, in which the posterior model structure is embedded, then to be consistent with all the prior assumptions that implementation of the filter necessitates, the following conditions would be required of $v\left(t_{k} \mid t_{k-1}\right)$ : (1) their mean values are zero (for each element of the vector sequence), (2) they are not significantly correlated with themselves in time, (3) they are not significantly correlated with any of the observed input sequences $\mathbf{u}$, and (4) their actual sample (posterior) variance-covariance properties are consistent with those expected (prior) estimates implied by the prior assumptions about the sources of uncertainty affecting the identification process (see, for example, Cosby and Hornberger [1984]).

If the posterior model satisfies these conditions, it is at least self-consistent, i.e., not in disagreement with those assumptions required for its identification. If it does not satisfy these conditions, especially in respect of conditions 2 and 3 above, then such results can be used to good effect for the purposes of model structure identification (as in the work by Beck [1978]).

\subsection{Model Discrimination}

The burden of the preceding discussion of residual error analysis essentially reduces to a problem of hypothesis testing, of accepting or rejecting the hypothesis that the actual statistical properties of the residuals are consistent with certain expected statistical properties. Hence a single posterior model structure resulting from the process of model structure identification and parameter estimation may be accepted or rejected as adequate. The same basic idea can be extended and reoriented for consideration of the problem of model discrimination, i.e., to compute summary statistics of the residual errors from several candidate model structures, then to rank these statistics and hence discriminate the best among several competing prior hypotheses about the behavior of the system. This latter is understood here as model discrimination. It has not been widely reported in the literature on water quality modeling, although it was in fact the objective of one of the first practical studies in parameter estimation to discriminate among various structures of steady state DO-BOD interaction models of the Sacramento River, California [Shastry et al., 1973]. A more recent, and much more comprehensive, analysis of model discrimination has been discussed by Cosby and Hornberger [1984], Cosby et al. [1984], and Cosby [1984]. They examine the problem of discriminating among eight (or five) possible expressions for the relationship between photosynthetic oxygen production and light intensity in the Gryde River, a macrophyte-dominated stream in Denmark. In a sense their results are not all that successful, because the competing model structures (except one, which incorporates an intuitively improbable linear expression) are capable of replicating more or less the same photosynthesis-light behavior by an appropriate adjustment of parameter estimates [Cosby et al., 1984]. It is difficult to discriminate among minor variations on the same theme. In another sense, better expressed as model structure identification, their results are highly productive. They observed that "... the proper structure must allow for temporary changes in the parameter values..." [Cosby et al., 1984] and were subsequently able to identify both a long-term and a short-term adaptivity of macrophyte photosynthetic behavior [Cosby, 1984].

\subsection{Commentary}

Interpretation of the past observed behavior of a system is never constant, and no analysis of field data is ever complete. 
There is an evolutionary nature to both. In the end it may not be the satisfaction of certain statistical measures that determines termination of the process of identification. Decisions are not delayed indefinitely; it is more than likely that the analysis will have to be terminated prematurely. Ideally then it ought to be the case that all the residual inadequacies of model structure, or lack of identifiable parameters, are properly accounted for in the application of the model for predictive purposes.

\section{Prediction Error Propagation}

The objectives for the analysis of uncertainty must now be considered in a broader context, that is, in light of what has been defined in the introduction as problem (P3): uncertainty associated with predictions of the future behavior of the system.

The emphasis here will be different from that of sections $3-6$, where the focus was exclusively on the use of models for the development of scientific theories about the behavior of complex environmental systems. Our discussion must now be increasingly concerned with the role of uncertainty in applying a model for the purposes of management, although this will not divorce it from the scientific issues already raised.

The essential questions for the analysis of prediction error propagation are those of (1) looking prospectively to the application of the model to management: how significant the prediction and its associated error are to the decisions that have to be made and if the options (for decision) can be meaningfully ranked against a background of inescapable uncertainty, and (2) looking in retrospect at the development of the model by reference to the historical observations: what the consequences are for prediction of a model that suffers from a lack of identifiability and, conversely, what can be concluded from the propagation of prediction errors about the different strategies of model development?

These, however, are general, perhaps philosophical, questions. The specific issues of concern are not as obvious as they might seem.

To begin with, inspection of the literature would suggest a great variety of methods for the quantitative analysis of prediction uncertainty. This is unnecessarily confusing. Insofar as can be established, there are just two basic methods, although one could argue about points of detail (see section 7.3). Second, the potential confusion may have been fueled by the broad and overlapping usage of terms such as sensitivity analysis, error analysis, and validation. Indeed, some of these difficulties, especially with the term validation, have already surfaced in the preceding section (section 6). Our working definitions of the relevant terms are therefore as follows.

Validation: given the model structure and parameter estimates, determine behavior under different observed input conditions for comparison of the output response with different observed behavior.

Conventional sensitivity analysis: given the model structure and parameter estimates, determine changes in the output model response that would result from changes in the estimated values of the parameters.

Prediction error propagation: given the model structure and parameter estimates, subject to uncertainty, determine future behavior under different (assumed) uncertain input conditions.
Reducing uncertainty: determine which sources of error (uncertainty) contribute most to the uncertainty of the predicted response and design experiments in order to reduce this uncertainty.

All of these issues are apparent in the early original contributions of the 1970's [Goodall, 1972; O'Neill, 1973; Miller, 1974; Burges and Lettenmaier, 1975; Miller et al., 1976; Argentesi and Olivi, 1976].

There is much that could be said on each issue. However, on validation we shall be brief. It can be regarded in the narrow formal sense defined above, as a quantitative method of evaluating an identified model. There have been few such analyses [Thomann et al., 1974; Jorgensen et al., 1978; Collins, 1980; Najarian et al., 1984], and they can largely be subsumed under what has been discussed here as the checks and balances of section 6 . Validation might otherwise be regarded in a much broader philosophical vein, almost as a vague concept (albeit intuitively understandable) that in practice is most useful in specifying procedures and protocols for model building [Cale et al., 1983a; Caswell, 1976; Holling, 1978; Mankin et al., 1977; Lewandowski, 1982; Loehle, 1983]. In this case it is conceivable that the whole of this review is about validation.

On conventional sensitivity analysis we shall be equally brief, narrowing its definition still further to analyses dependent only on the solution of what will be denoted in section 7.3 as the sensitivity equations (see, for example, the case studies of Halfon [1977], Rinaldi and Soncini-Sessa [1978], van Straten and de Boer [1979], and Najarian and Taft [1981]; see also the substantial literature of chemical engineering [McCrae, 1987]). In this restricted sense the difference between conventional sensitivity analysis and a first-order error analysis (see section 7.3) is that the former makes no reference to the sources of uncertainty that might affect the outcome of the predicted state of the system; it involves no concept of the uncertainty of a prediction.

It is thus specifically the questions of, How confident is the prediction?, What are the principal sources of the uncertainty of the prediction?, and How can these uncertainties be reduced?, that are of greatest interest.

\subsection{Prediction After Identification}

Irrespective of the strategy adopted in developing a model, it is essentially the knowledge and uncertainty of the internal description of the system's behavior that is changed by the process of identification. In other words, referring to Figure 1, the knowledge of the states (x) and parameters $(\boldsymbol{\alpha})$, the structure of their interrelationships, estimates of their values, and estimates of their uncertainty (error variance-covariance properties) have been modified. The (prior) assumptions about the uncertainty in the external description of the system, namely, the uncertainty associated with the measured and unmeasured input disturbances $(\xi)$ and the output response observations ( $\eta$ ), do not necessarily have to be changed in so significant a manner. The same assumptions about these latter might also be made for the purpose of prediction, although it is quite possible that the process of identification may establish their inadequacy and prompt improved (posterior) assumptions.

In this review, the a posteriori residual errors of mismatch between the observed and estimated output responses, i.e., $(\mathbf{y}-\langle\mathbf{y}\rangle)$ (sometimes referred to as the "model errors") are not 
considered to be carried forward from identification to the analysis of prediction error propagation. They are regarded as a by-product of identification, as Figure 1 suggests. It is quite possible, of course, that prediction errors can be quantified in like terms, but this is achieved simply by transforming the future state $(\mathbf{x})$, parameter $(\boldsymbol{\alpha})$, and other errors $(\boldsymbol{\xi}$, and where appropriate $\boldsymbol{\eta}$ ) into an error in the output response variable (y). There are clearly cases in which it might be appropriate to quantify the error of predicted future behavior directly as a function of these residual errors of mismatch, one example being the application of Vollenweider's phosphorus-loading models reported by Reckhow and Chapra [1983b]. However, interpretation of the residual errors in this manner leads to certain conceptual difficulties, for they are an amalgam of both the various (prior) assumptions about the sources of uncertainty and the consequences of the process of identification. For the purpose of analyzing prediction error propagation our preference here is to maintain a procedure of accounting strictly for the individual sources of uncertainty, for assessing the effects of identification on these uncertainties, and ultimately assessing the effects of the individual uncertainties on predictions of the future. So it is the a posteriori parameter (and state) estimation errors that provide the crucial logical link that we wish to establish between identification and prediction.

In simple terms the results of the whole process of identification and the consequences of a lack of identifiability are crystallized in the a posteriori parameter estimation error variance-covariance matrix, $P^{P}\left(t_{N}\right)$. Qualitatively, well (or poorly) estimated parameters will have relatively low (or high) error variances; parameters associated with redundant hypotheses should be highly uncertain; and parameters associated with multiple hypotheses, the collective effects of which cannot be disentangled, should yield significant off-diagonal covariances in the matrix $P^{P}\left(t_{N}\right)$ [e.g., Di Toro and van Straten, 1979; van Straten, 1983; Young et al., 1980]. It is intuitively obvious in this last case that many combinations of parameter values will give equally good matches between the model and past field observations; there is ambiguity in the identified model. Questions must then be resolved as to which combinations of parameter values should be used for prediction and whether, in the face of prediction uncertainty, there is any significant difference between alternative statements about future behavior. The argument has its parallel in the context of the HSY algorithm discussed in section 3 [Fedra et al., 1981]. The ensemble of behavior-generating parameter values $\alpha^{*}(B)$ represents an ensemble of equally probable models for the description of past behavior. In turn therefore each such set of values for the parameter vector is an equally likely candidate for making a prediction of future behavior and, providing there is a sufficiently large sample for $\alpha^{*}(B)$, corresponding distributions of the predictions can be generated and analyzed.

\subsection{Alternative Conceptual Frameworks}

The conceptual framework of control theory, upon which the preceding arguments have been constructed, does not necessarily accommodate most readers' views of the analysis of prediction error propagation. It is not altogether in accord with the statistical view of uncertainty, identification, and prediction, as illustrated, for example, by Reckhow and Chapra [1983a, b] and Reckhow [1983]. And it is different again from the perspective of systems ecology that underpins the work of O'Neill and Gardner and their colleagues. The two frameworks (of control theory and systems ecology) are not incompatible, but some translation is necessary.

O'Neill's [1973] original paper on the subject of error analysis noted that the error in the predictions from a model should decrease with a decreasing degree of model aggregation (in an ecological, as opposed to spatial or temporal, sense in this particular case). However, he noted also that precisely this increasing refinement of detail-more complex kinetic expressions, more state variables-would tend to increase the prediction errors resulting from the necessarily increasing number of model parameters with uncertain values. For instance, errors of $10 \%$ (expressed as a coefficient of variation) in the parameter values were found to yield errors of prediction of greater than $100 \%$. The exploration of such problems naturally bears upon the theoretical foundations of the analysis of uncertainty, and this form of enquiry has been a distinctive feature of the work of O'Neill and Gardner and their associates. Many of their results are reviewed in the work by O'Neill and Gardner [1979], where they classify the sources of uncertainty into the following categories (for comparison with Figure 1).

1. Uncertainty resulting from model construction, composed of model aggregation (see also O'Neill and Rust [1979], Cale and Odell [1979], Gardner et al. [1982], Cale et al. [1983b], and Halfon and Maguire [1983]), alternative models, and model structure, all of which would be covered by the definition of the errors of the a priori assumptions about the internal description of the system in Figure 1.

2. Uncertainty resulting from parameter estimation errors (see also O'Neill et al. [1980], Gardner et al. [1980a, b, 1981a], and Gardner and O'Neill [1983]).

3. Uncertainty resulting from natural variability, comprising (1) environmental variability, which can be equated with uncertainty in the system input disturbances (see also Somlyody [1983]), (2) spatial heterogeneity (see also O'Neill et al. [1979] and Gardner et al. [1981b]), which according to Figure 1 would be defined under aggregation or lumping errors, and (3) genetic variability (see also O'Neill [1979]), which in practice would be indistinguishable from the errors of parameter estimation (see also, for further discussion, Gardner and $O^{\prime}$ Neill [1983]).

The group's principal analytical results refer to the analysis of (spatial and ecological) aggregation error and to the errors deriving from genetic variability as it affects simple kinetic expressions for growth rate and other functional aspects of ecological systems. These, however, since they require a detailed knowledge of ecological interactions, will be considered to be outside the scope of the review. Nevertheless, many of the group's conclusions are of central importance to the subsequent discussion, especially those relating to the effects of parameter uncertainty on prediction error propagation and to the comparative advantages and disadvantages of Monte Carlo simulation and first-order error analysis. One observation, in particular, will set the scene for what follows.

Although interesting analytical results have been achieved with linear and simple nonlinear models, the real challenge lies with complex ecosystem models that have been developed over the past decade.... Investigation of error propagation in these large 
nonlinear models will have to be inductive, with numerous individual applications required before general patterns begin to emerge.

\section{[O'Neill and Gardner, 1979].}

\subsection{Approaches and Methods}

In its most complete form, an analysis of the propagation of state and parameter prediction uncertainty (or error) would require computation of the evolution with time of the entire probability density function of $\left\langle\mathbf{x}_{a}\left(t \mid t_{f}\right)\right\rangle$ for $t \geq t_{f}$, where $t_{f}$ is the time at the beginning of the prediction period. Here the notation $\left\langle\mathbf{x}_{a}\left(t \mid t_{f}\right)\right\rangle$ indicates that in principle the predictions are conditional upon field observations available up to time $t_{f}$, although from here on this conditional nature of the prediction will be taken as implicit to simplify notation, i.e., $\left\langle\mathbf{x}_{a}(t)\right\rangle \equiv\left\langle\mathbf{x}_{a}\left(t \mid t_{f}\right)\right\rangle$. To be complete in the computation of error propagation would require solution of a Fokker-Plank or Kolmogorov forward equation [e.g., Jazwinski, 1970]. Occasional reference to this requirement has been made in studies of the application of stochastic differential equations in stream water quality modeling [Finney et al., 1982] and wastewater treatment plant modeling [Harris, 1977]. But just as with the Bayesian estimation problem of section 5, for which the same kinds of detailed knowledge are also required, the computational effort and complication of deriving such solutions for the general nonlinear (class II) model of (32) are formidable. Again therefore the specific assumption of Gaussian probability density functions, or simply the pragmatic restriction of interest to the first and second moments of any more general probability density function (i.e., the mean and variance-covariance statistics, respectively) can lead to more tractable solutions.

Of the two dominant approaches to the analysis of prediction error propagation, the above restrictions refer primarily to what is known as a first-order (or, more rarely, secondorder) error/sensitivity analysis. They may, of course, be pertinent to the other principal approach, that of the ubiquitous Monte Carlo simulation, but in this second case they are not crucial to its application. We may note in passing that Benjamin and Cornell [1970] have discussed Monte Carlo simulation as an approximate numerical technique for solving the problem of derived distributions, which is essentially the same problem as that introduced here, i.e., deriving (analytically) the entire probability density function of the predicted variables.

A definition of the approach of Monte Carlo simulation follows from $(32 a)$, i.e.,

$$
d \mathbf{x}(t) / d t=\mathbf{f}\{\mathbf{x}, \mathbf{u}, \boldsymbol{\alpha} ; t\}+\xi(t)
$$

The procedure is simply to generate a sample set of realizations $\mathbf{x}^{i}(t)$ over the prediction period $t_{f} \leq t \leq t_{p}$, where each such realization, denoted by superscript $i$, is computed from (34) with the specific choices of values for the initial state $\mathbf{x}^{i}\left(t_{f}\right)$, the parameters $\alpha^{i}(t)$, the measured input disturbances $u^{i}(t)$, and the sequence of errors $\xi^{i}(t)$ drawn at random from (estimates of) their respective parent probability density functions. The specific procedure of drawing samples from the population can be implemented in a number of ways. There are nonrandom procedures, classified under the title of stratified end pattern search methods [McCrae, 1986], and the Latin Hypercube sampling strategy, in particular, has already been illustrated by Jaffe and Ferrara [1984] and Jaffe and Parker [1984]. Though the vast literature on experimental design to which this alludes must clearly be acknowledged, in this paper no such distinctions will be made in referring generally to the approach of Monte Carlo simulation. The number of sample realizations (i) required to approximate the population statistics to a sufficient degree of accuracy can either be determined by using the Kolmogorov-Smirnov and Renyi statistics [Spear, 1970; Spear and Hornberger, 1980] or, more arbitrarily, by experience (Gardner et al. [1980a] quote a figure of 500 samples as being usually adequate). The evolution of the entire distribution for the state vector $\mathbf{x}(t)$ is thus computed, and in this sense, Monte Carlo simulation is, as Dettinger and Wilson [1981] have referred to it, a full distribution technique.

First- and higher-order error (sensitivity) analyses of the predictions generated by the model of (34) depend essentially on the conceptual separation of the problem into the two subproblems of computing a nominal (mean) reference trajectory for the future state of the system and of assessing the effects of the various small-amplitude sources of uncertainty on the uncertainty of that trajectory. If we restrict attention to a first-order error analysis, which reflects almost entirely the attention of the associated literature, the nominal reference prediction $\langle\mathbf{x}(t)\rangle$ of the future state $\mathbf{x}(t)$ is then given by (from 34))

$$
d\langle\mathbf{x}(t)\rangle / d t=\mathbf{f}\{\langle\mathbf{x}\rangle,\langle\mathbf{u}\rangle,\langle\boldsymbol{\alpha}\rangle ; t\}+\langle\boldsymbol{\xi}(t)\rangle
$$

Here $\langle\mathbf{x}(t)\rangle$ can be considered to be the estimated mean (first moment) of the distribution of $\mathbf{x}(t)$, and likewise $\langle\mathbf{u}(t)\rangle,\langle\boldsymbol{\alpha}(t)\rangle$, and $\langle\xi(t)\rangle$ can be regarded as the estimated or assumed means of their respective distributions. Equation (35) is directly analogous to the reference model of ( $7 a)$ discussed in respect of the problem of model structure identification in section 4 .

There are two approaches to the linearization procedure inherent in a first-order error analysis, and these lead to different formulations for computing the propagation of $P^{s}(t)$, the variability, or uncertainty, or error variance-covariance (second moment) of the predicted state $\langle\mathbf{x}(t)\rangle$. In one of these the connection with conventional sensitivity analysis is clearly apparent; the other owes its allegiance much more to the notions of an error propagation model and recursive estimation as already discussed in section 4 . In either case it is not necessary to compute the propagation of the prediction errors themselves but merely to quantify the propagation of their variance-covariance properties as a function of the variancecovariance properties of the contributing sources of uncertainty, namely, the following.

1. $E\left\{\tilde{\mathbf{x}}\left(t_{f}\right) \tilde{\mathbf{x}}^{T}\left(t_{f}\right)\right\}=P^{s}\left(t_{f}\right)$, representing the uncertainty in the initial state of the system.

2. $E\left\{\tilde{\mathbf{u}}(t) \tilde{\mathbf{u}}^{T}(t)\right\}=S(t)$, representing uncertainty in the measured inputs, a source of uncertainty previously subsumed under the definition of source 4 , below.

3. $E\left\{\tilde{\boldsymbol{\alpha}}(t) \tilde{\boldsymbol{\alpha}}^{T}(t)\right\}=P^{P}(t)$, representing parameter uncertainty, and perhaps most conveniently assumed to be equal to $P^{P}\left(t_{N} \mid t_{N}\right)$ at the end of some prior identification process.

4. $Q^{s}(t)$ as the variance-covariance matrix of the (fictional) continuous-time white noise process $\xi(t)$ representing uncertainty associated with unmeasured input disturbances and all other undifferentiated sources of uncertainty.

Here $\tilde{\mathbf{x}}$, $\tilde{\mathbf{u}}$, and $\tilde{\alpha}$ are the errors (or small perturbations) previously defined in section 4 by (8). They would normally be assumed to have zero mean values, as would $\langle\xi(t)\rangle=\mathbf{0}$, and the parameters would normally be further assumed to be time invariant.

The first approach to the computation of $P^{s}(t)$, which is the more commonly encountered, derives much more directly 
from sensitivity analysis. It expresses the instantaneous value of $P^{s}(t)$ at future time $t$ as a function of the variancecovariance matrices $P^{s}\left(t_{f}\right), S(t), P^{p}(t)$, and $Q^{s}(t)$ and of the sensitivity coefficient matrices with respect to $\mathbf{x}(t)$ evaluated at time $t$ and as a function of $\langle\mathbf{x}(t)\rangle$. Here the sensitivity coefficients derive from a linearization about the nominal reference prediction $\langle\mathbf{x}(t)\rangle$, and in order to obtain the values of these sensitivity coefficients at time $t$ it is necessary to integrate an additional set of ordinary differential equations for the evolution of the sensitivity coefficients with time. Accordingly, $P^{s}(t)$ is given by (see also Burns [1975] and Argentesi and Olivi $[1976])$

$$
\begin{aligned}
P^{s}(t)=B^{0}(t) P^{S}\left(t_{f}\right)\left[B^{0}(t)\right]^{T}+B^{P}(t) P^{P}(t)\left[B^{P}(t)\right]^{T} \\
+B^{u}(t) S(t)\left[B^{u}(t)\right]^{T}+B^{\xi}(t) Q^{s}(t)\left[B^{\xi}(t)\right]^{T}
\end{aligned}
$$

where the sensitivity coefficient matrices $B^{0}(t), B^{P}(t), B^{u}(t)$, and $B^{\xi}(t)$, having elements $\left[\partial x_{i}(t) / \partial x_{j}\left(t_{f}\right)\right],\left[\partial x_{i}(t) / \partial \alpha_{j}\right],\left[\partial x_{i}(t) / \partial u_{j}\right]$, and $\left[\partial x_{i}(t) / \partial \xi_{j}\right]$, respectively, are obtained from the following differential equations:

$$
\begin{array}{cc}
d B^{0}(t) / d t=F_{11}(t) B^{0}(t) & B^{0}\left(t_{f}\right)=I \\
d B^{P}(t) / d t=F_{11}(t) B^{P}(t)+F_{12}(t) & B^{P}\left(t_{f}\right)=0 \\
d B^{u}(t) / d t=F_{11}(t) B^{u}(t)+G(t) & B^{u}\left(t_{f}\right)=0 \\
d B^{\xi}(t) / d t=F_{11}(t) B^{\xi}(t)+I & B^{\xi}\left(t_{f}\right)=0
\end{array}
$$

Here the matrices $F_{11}, F_{12}$, and $G$ contain elements $[i, j]$ defined by the derivatives $\left[\partial f_{i}\{\cdot\} / \partial x_{j}\right],\left[\partial f_{i}\{\cdot\} / \partial \alpha_{j}\right]$, and $\left[\partial f_{i}\right.$ $\left.\{\cdot\} / \partial u_{j}\right]$, respectively, where these derivatives are evaluated using the nominal reference values $\langle\mathbf{x}(t)\rangle,\langle\boldsymbol{\alpha}(t)\rangle$, and $\langle\mathbf{u}(t)\rangle$. The sensitivity equations $(36 b)-(36 e)$ are, strictly speaking, exact only for the case where the variations of $\alpha, \mathbf{u}$, and $\xi$ with time are zero [Eykhoff, 1974], a point to which we shall return below. They are the equations of conventional sensitivity analysis, and it is from the solution of $(36 c)$ in particular that the results of Figure 2 have been generated for an assessment of model identifiability (see section 2). A related matrix of sensitivity coefficients has also appeared earlier in the determination of the posterior parameter estimation error variances given by (31) of section 5 .

The second approach to the computation of $P^{s}(t)$ follows directly from the discussion of the linear model of error propagation discussed in section 4, and it may be helpful to note that Malone et al. [1984] have referred to this approach as a "generation of moment equations" method. In fact, given our preceding discussion of recursive estimation, the algorithm (including (35) above) is equivalent to a partitioned extended Kalman filter from which the correction step has been removed (or alternatively, the filter can be imagined to be processing data expected to arrive at an infinite time horizon). In this case the linearization is made about the derivative of the augmented state vector, i.e., the function $\mathbf{f}_{a}\{\cdot\}$, which equals $d \mathbf{x}_{a}(t) / d t$. An expression for the propagation of $P^{s}(t)$ can then be derived directly from the (integrated) discrete-time difference-equation form of the error propagation model, i.e., $(10 a)$. This is achieved simply by setting up the relevant expression for $\tilde{\mathbf{x}}_{a}\left(t_{j+1}\right) \tilde{\mathbf{x}}_{a}{ }^{T}\left(t_{j+1}\right)$ and then applying the operation of mathematical expectation [Beck et al., 1979; Schweppe, 1973]. In partitioned form, that is, distinguishing between $\tilde{\mathbf{x}}$ and $\tilde{\alpha}$ within the augmented state vector errors $\tilde{\mathbf{x}}_{a}$, the following recursive algorithm results:

$P^{s}\left(t_{j+1}\right)=\Phi_{11}\left(t_{j+1}, t_{j}\right) P^{s}\left(t_{j}\right) \Phi_{11}{ }^{T}\left(t_{j+1}, t_{j}\right)$

$$
\begin{aligned}
& +\Phi_{11}\left(t_{j+1}, t_{j}\right) P^{c}\left(t_{j}\right) \Phi_{12}{ }^{T}\left(t_{j+1}, t_{j}\right) \\
& +\Phi_{12}\left(t_{j+1}, t_{j}\right)\left[P^{c}\left(t_{j}\right)\right]^{T} \Phi_{11}{ }^{T}\left(t_{j+1}, t_{j}\right) \\
& +\Phi_{12}\left(t_{j+1}, t_{j}\right) P^{P}\left(t_{j}\right) \Phi_{12}{ }^{T}\left(t_{j+1}, t_{j}\right) \\
& +\Gamma\left(t_{j+1}, t_{j}\right) S\left(t_{j+1}, t_{j}\right) \Gamma^{T}\left(t_{j+1}, t_{j}\right)+Q^{s}\left(t_{j}\right)
\end{aligned}
$$

where $P^{c}\left(t_{j}\right)$ is the matrix of state-parameter error covariances and is propagated according to

$P^{c}\left(t_{j+1}\right)=\Phi_{11}\left(t_{j+1}, t_{j}\right) P^{c}\left(t_{j}\right)+\Phi_{12}\left(t_{j+1}, t_{j}\right) P^{P}\left(t_{j}\right)$

and

$$
P^{P}\left(t_{j+1}\right)=P^{P}\left(t_{j}\right)+Q^{P}\left(t_{j}\right)
$$

Here the integration interval is from $t_{j}$ to $t_{j+1}$, and the matrices $\Phi_{11}, \Phi_{12}$, and $\Gamma$, where $\Phi_{11}$ and $\Phi_{12}$ are submatrices of $\Phi$, refer to the discrete-time error model of $(10 a)$. These matrices in turn depend upon the matrices $F_{11}, F_{12}$, and $G$ as defined above for the companion algorithm of (36), which themselves refer to the continuous-time error propagation model of $(9 a)$. The matrix $Q^{P}$ in $(37 c)$ has also been defined earlier in section 4 as the variance of the random perturbations affecting the temporal evolution (if any) of the model parameters (a matter of seemingly little importance, although possibly of vital significance to the discussion in section 7.4). An analogous derivation of the algorithm for the case of a partial-differential equation (class I) model has been presented by McLaughlin [1983].

The complete algorithm for the mean and error variancecovariance matrix of the prediction using a first-order error analysis is therefore given by (35) and either (36) or (37). The astute reader will have noticed that there are discrepancies between the two alternatives, with (37) appearing to be the less restrictive in its assumptions, and the more complete by its inclusion of $P^{c}$ and $Q^{P}$.

This is indeed probably more apparent than real. Were the relationships of (36) to be derived from an augmented system of dynamical equations for $\{\mathbf{x}, \boldsymbol{\alpha}, \mathbf{u}$, and $\boldsymbol{\xi}\}$, subsequently partitioned, and then suitable assumptions made about certain unlikely error covariances, e.g., between $\tilde{\mathbf{x}}(t)$ and $\tilde{\mathbf{u}}(t)$, it would be reasonable to conjecture that the two alternatives would be identical (subject to identical intervals of integration). Presentation of the algorithms for $P^{s}(t)$ in the given forms is a thoroughly pragmatic reflection of their most usual forms of application.

We shall likewise pass over the rarely used second-order error analysis without further comment, except for the following point [see, for example, Dettinger and Wilson, 1981]. In the second-order approximation the mean value of the state prediction, for the scalar case of errors in a single parameter $\alpha$ only, is given by

$$
\begin{aligned}
d\left\langle x^{(2)}(t)\right\rangle / d t=f\left\{\left\langle x^{(2)}\right\rangle,\langle u\rangle,\langle\alpha\rangle ; t\right\} & +\langle\xi(t)\rangle \\
& +(1 / 2) f^{(2)}(t) p^{P}(t)
\end{aligned}
$$

where $f^{(2)}(t)$ is the second derivative of $f\{\cdot\}$ with respect to $\alpha$, $p^{p}(t)$ is the variance of the estimation errors of $\langle\alpha\rangle$, and $\left\langle x^{(2)}(t)\right\rangle$ is the mean predicted value of $x(t)$ in the second-order approximation. By comparison with (35) it is clear that the first-order approximation of the mean is exact when $f^{(2)}(t)=0$, i.e., $f\{\cdot\}$ is linear in the parameter $\alpha$. It is also apparent that when $f^{(2)}(t) \neq 0$, significant errors in the parameters will cause the first-order mean to $t_{-}$biased, and that the degree of nonlinearity of $\alpha$ in $f\{\cdot\}$ is reflected in the magnitude of $f^{(2)}(t)$. Inherent in these observations on (38) are thus some of the limitations that have been the principal subject of discussion 
in the comparative studies of first-order analysis and Monte Carlo simulation.

So to summarize, a first-order error analysis involves computation of two statistics of the distribution of the errors $(\tilde{\mathbf{x}})$ in the predicted state (about a nominal, deterministic reference prediction, $\langle\mathbf{x}\rangle$ ). Monte Carlo simulation permits analysis (by whatever appropriate statistics) of a sample approximation of the distribution of possible future realizations of the state $\mathbf{x}$.

\subsection{Case Studies}

7.4.1. Prediction error magnitudes. The extension of conventional sensitivity analysis to the first-order analysis of prediction error propagation appears to have been first proposed for water quality/ecological models by Burges and Lettenmaier [1975] and Argentesi and Olivi [1976]. It has since been applied by many authors, both in the form of (36) [Lettenmaier and Richey, 1979, Reckhow, 1979a; Di Toro and van Straten, 1979; van Straten, 1983; Chadderton et al., 1982; Walker, 1982] and in the form of (37) [Scavia, 1980; Scavia et al., $1981 a, b ;$ Beck, 1981a, 1983]. Nearly all these studies have focused on the problem of lake eutrophication, with the models analyzed ranging from the single response-variable model of Vollenweider [Reckhow, 1979a, b; Walker, 1982] to a 16-state variable model for the carbon-, nitrogen-, and phosphorus-cycle dynamics in Lake Ontario [Di Toro and van Straten, 1979; van Straten, 1983].

It is unlikely that these were studies motivated by a desire to demonstrate just how uncertain a prediction can be, but it is instructive to review the figures quoted for the levels of this uncertainty. Argentesi and Olivi's analysis of a three-state variable phytoplankton model for Lake Endine, Italy, showed that a $10 \%$ error on the model parameters, initial states, and (input) solar radiation pattern could lead to prediction errors well above $100-200 \%$ during certain periods of the annual cycle. Van Straten's [1983] results for the model of Lake Ontario likewise underline the considerable magnitude of the errors in predictions that might be obtained: of up to $2050 \%$, for example, although the parameter errors relevant to this analysis are correspondingly of a high order, with individual values exceeding $1000 \%$. The results of the analysis of Scavia et al. [1981a] of a version of the same Lake Ontario model adapted for the inner portion of Saginaw Bay, Lake Huron, are similar to those of van Straten. For instance, for parameter, initial state, and input disturbance error levels ranging between $24 \%$ and $202 \%$, maximum prediction errors of between $148 \%$ and $772 \%$ were obtained. Very much the same sort of results have also been obtained by M. B. Beck and E. Halfon (manuscript in preparation, 1986) in their analysis of (yet another) model for Lake Ontario. In the analyses of both van Straten and Scavia et al. the highest prediction error levels were associated with state variables representing zooplankton concentrations.

The general pattern of the results emerging from the use of Monte Carlo simulation, at least simply in terms of the magnitudes of the prediction errors propagated, is not substantially different from that of the first-order error analyses [e.g., Burges and Lettenmaier, 1975; Hornberger, 1980; O'Neill et al., 1980; Gardner et al., 1980a, 1981a; Gardner and O'Neill, 1983; Scavia et al., 1981b; Fedra et al., 1981; Walker, 1982; Somlyody, 1983].

Thus if the point has to be made, there is ample evidence to suggest that the currently available models of water quality, and in particular the larger models, give predictions that are highly uncertain (with coefficients of variation upward of $700 \%$, if such a statistic then has any real meaning). To be able to predict only that all things are more or less equally probable is not a useful basis for decision making. But there are certain factors, relating specifically to the possible consequences of identifiability, that will tend to counterbalance the generation of such enormous prediction error levels: these are the effects of covariance (correlation) among the estimation errors of the model parameter values. For instance, in van Straten's case study of Lake Ontario, it was possible to reduce the prediction errors by over an order of magnitude when correlations among the parameter estimation errors (with correlation coefficients of up to $0.8-0.9$ ) were taken into account. Other examples are less dramatic [O'Neill et al., 1982a; Beck, 1983] but strongly supportive of van Straten's results. In fact it is worth noting in passing that the approach of O'Neill et al. is in this case closely similar to some of the ideas introduced by the HSY approach in section 3 .

7.4.2. Comparative studies. If the errors in the model parameters, initial states, and input disturbances are large (as indeed they are in some of the above studies), then this is a blatant violation of the key assumption in a first-order error analysis that all these errors should be small. There has therefore been widespread concern with the question of how the performances of Monte Carlo simulation and a first-order error analysis compare [Burges and Lettenmaier, 1975; Gardner et al., 1981a; Gardner and O'Neill, 1983; Scavia et al., $1981 b ;$ Walker, 1982; Malone et al., 1984]. Thus Burges and Lettenmaier conclude from an analysis of a stream DO-BOD model, with the three model parameters having coefficients of variation of $25 \%, 29 \%$, and $32 \%$, that the predicted means from the two methods are "not appreciably different" and the variances of the prediction errors from the first-order analysis are "quite satisfactory." Walker [1982] concludes likewise that the two methods "compare reasonably," and Malone et al. [1984] observe that "for all practical purposes the ... techniques produced identical results" for their case study of a simple phosphorus model for Lake Washington.

The conclusions drawn from the comparative analyses of Scavia et al. [1981a], Gardner et al. [1981a], and Gardner and O'Neill [1983] are, however, otherwise. They suggest both significant and subtle differences of outcome from the two approaches. Although Scavia et al. [1981b] note that the two estimates of prediction error variance propagation are in general "qualitatively similar," there are (from time to time) quite specific differences due, in their opinion, to the following three causes.

1. The mean values, $\langle\mathbf{x}(t)\rangle$, derived from the sample set of realizations of (34), i.e., the mean of the Monte Carlo simulations, are not identical with the ("deterministic") mean values derived from (35) (see also the discussion of (38)).

2. The first-order approximation deriving from the linearization procedure in a first-order analysis is a poor approximation in the presence of strong nonlinearities and large errors (see again the discussion of (38)).

3. The error variance estimate from the Monte Carlo simulations is difficult to interpret, or ambiguous, if the sample frequency distributions exhibit significant skewness or are bimodal, i.e., the first and second moments poorly characterize the full distribution.

The effects of these three items in the study of Scavia et al. [1981b] are clearly not negligible. For example, Figure 13 shows the significant differences between the estimated predic- 


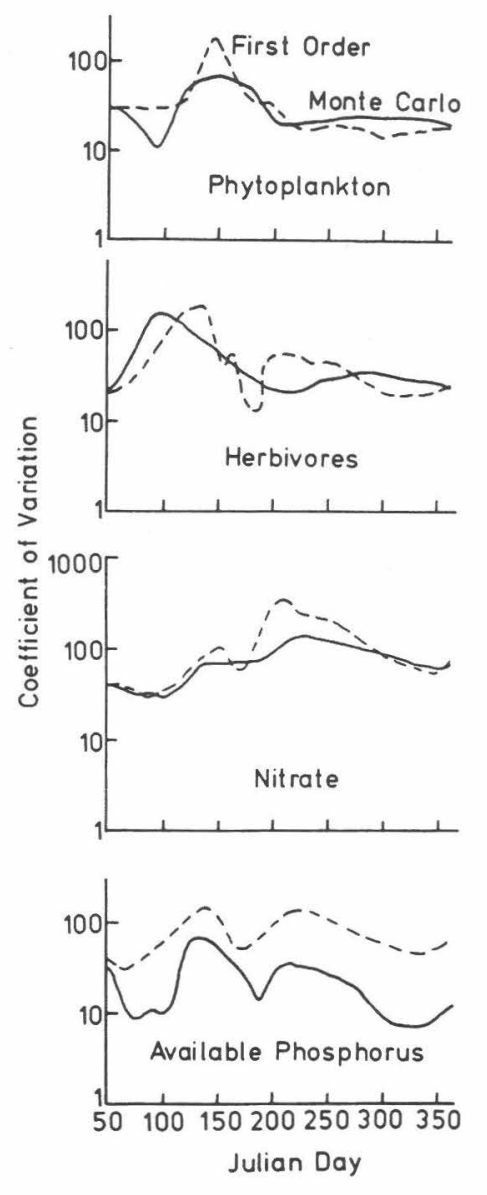

Fig. 13. Comparative study of the use of first-order error analysis (dashed lines) and Monte Carlo simulation (solid lines) with a model for Saginaw Bay, Lake Huron [after Scavia et al., 1981b]. The magnitudes of the errors of prediction are expressed as coefficients of variation (in percent).

tion error variances expressed as coefficients of variation, although elsewhere, Scavia has given results supporting the strong similarity of first-order and second-order approximations, which would tend to lessen the significance of point 2 above [Scavia, 1980]. In short, Scavia et al. are unable of resolve entirely the differences between the results from Monte Carlo simulation and first-order error analysis and conclude that the interpretation of the error variances from the two approaches should be considered fundamentally different. They argue, in support, that the first-order analysis refers to predictions about the future behavior of a typical representative (species) of a (biological) population, whereas Monte Carlo simulation refers to the population as an ensemble.

Gardner and O'Neill's [1983] conclusions are yet again somewhat different. They state that while "...the assumptions of sensitivity [first-order error] analysis do not appear to cause serious problems..." there is a danger that such analysis may prompt significantly misleading inferences about solutions to the problem of reducing prediction uncertainty.

7.4.3. Kremer's results. The overall impression now emerging is that prediction error variances can be very large and that the approach of Monte Carlo simulation, with its lack of restrictive assumptions, would in general be preferred. Kremer's [1983] results run against the trend of both these provisional observations and in an especially provocative manner.
The basis of Kremer's study is that the model parameters $(\alpha)$ can be assumed to vary with time in a random fashion. This is entirely consistent with the model assumed for the parametric variations in the discussion of recursive estimation (specifically $(6 a)$ of section 4). But it is clearly different from the normal assumption of Monte Carlo simulation. Kremer argues that his assumption has a certain biological validity (in relation to the natural genetic variability in a population of organisms). Hence a random choice of parameter values at each point in time, i.e., $\left\langle\alpha\left(t_{j} \mid t_{f}\right)\right\rangle \neq\left\langle\alpha\left(t_{f} \mid t_{f}\right)\right\rangle$ in general, leads to state variable predictions with a relatively small variance and with future possible trajectories clustered relatively closely around the nominal reference trajectory (i.e., of (35) in a firstorder error analysis). For his simple model of competitive algal growth, this contrasts with the results from a sample of random choices of the initial values of the parameters only, i.e., $\left\langle\boldsymbol{\alpha}\left(t_{j} \mid t_{f}\right)\right\rangle=\left\langle\boldsymbol{\alpha}\left(t_{f} \mid t_{f}\right)\right\rangle$ in any single-prediction trajectory, which are distinctly bimodal and thus suggestive of a high prediction error variance. The underlying mechanism of the difference is that (chance) extreme values of the parameters do not persist for all time when $\langle\boldsymbol{\alpha}\rangle$ is allowed to vary randomly with time.

This undoubtedly has important implications, not only for the way in which the results from the majority of studies using Monte Carlo simulation should be interpreted, but also for the use of first-order error analysis. In the algorithm of (37), the case of temporally varying parameters can be accommodated via the variance matrix $Q^{P}$, representing the expected value of the variance of the integral of the stochastic disturbance rate process $\zeta(t)$ over the interval $\Delta t=t_{j+1}-t_{j}$. Were the parameters to vary rapidly with time, never being persistently biased for any significant period of time, then one might expect $Q^{P} \simeq 0$ (perhaps counterintuitively, but the net effect of their fluctuations would be zero). Should they, on the other hand, vary slowly (with respect to the interval $\Delta t$ ), they might assume persistently extreme values over some period, and conversely, $Q^{P}$ should be relatively large. Such an argument is purely speculative, but it is the only interpretation in agreement with Kremer's findings that the "faster" the variation in the parameter values, the smaller the variance of the prediction errors. And this, too, is a conclusion of possibly still greater significance, as we shall observe later.

7.4.4. Ranking the sources of uncertainty. Let us turn first, however, to the question of establishing the relative importance of the various sources of uncertainty to the propagation of prediction errors [Miller et al., 1976; Argentesi and Olivi, 1976; Kohberger et al., 1978; O'Neill et al., 1980; Gardner et al., 1980a, 1981a; Scavia et al., 1981a; Halfon, 1984; Gentil and Perrier, 1985]. In the context of Figure 1, the objective is to rank the contributions to the error of a prediction from four sources: the initial state of the system, the parameter estimates of the model, the measurements of the input (and output) variables, and the unknown input disturbances of the system, or the model structure, or whatever other factors are considered to be defined conceptually in the term $\xi$ in the basic model of (34).

Having satisfied this more strategic objective, it will probably then be desirable, as a secondary objective, to rank the contributions of the individual parameters or variables to each of the four primary sources of uncertainty.

From a first-order error analysis and for the special case where all the parameters, say, have identical error levels (coefficients of variation) and there is no correlation among these 
parameter errors, the relative significance of each individual error can be ranked straightforwardly as a function of the relative sensitivity coefficient defined as

$$
\bar{b}_{i j}{ }^{p}(t)=\left[\partial x_{i}(t) / \partial \alpha_{j}(t)\right] /\left[\left\langle x_{i}(t)\right\rangle /\left\langle\alpha_{j}(t)\right\rangle\right]
$$

at time $t$. In order to obtain an aggregate ranking for the entire prediction period $t_{f} \rightarrow t_{p}$ it would be necessary to integrate $\bar{b}_{i j}{ }^{p}(t)$ accordingly and to rank the resulting integrals. For the rather more general case where the parameter errors do not have identical levels, yet are not correlated, the percentage contributions $\left(c_{i j}\right)$ of the error on parameter $j\left(p_{j j}{ }^{p}\right)$ to the error variance of state $i\left(p_{i i}{ }^{s}\right)$ can be computed from [Argentesi and Olivi, 1976]

$$
c_{i j}=100\left\{\int_{t_{j}}^{t_{p}}\left[\partial x_{i}(t) / \partial \alpha_{j}(t)\right]^{2} p_{j j}^{p}(t) d t\right\} /\left\{\int_{t_{f}}^{t_{p}} p_{i i}{ }^{s}(t) d t\right\}
$$

Both (39) and (40) are based on (36) as the approach to the variance-covariance computation of a first-order error analysis.

The ranking of the relative significance of the individual sources of error can also be posed as a question of the degree of correlation between the predicted state errors and the parameter errors. It is in fact this interpretation, and the direct use of the state-parameter covariance matrix $P^{c}$ from $(37 b)$, when normalized in the form of correlation coefficients, that Scavia et al. [1981a] adopt for their analysis of a model for Saginaw Bay, Lake Huron. The same conceptual approach to the ranking problem is taken by those studies using Monte Carlo simulation for the analysis of prediction error propagation. For instance, O'Neill et al. [1980] and Gardner et al. [1980a] generate correlation coefficients between variations in the sample set of predictions $\mathbf{x}^{i}(t)$ and variations in the random realizations of the sample set of parameter values $\alpha^{i}(t)$ (see also Gardner et al. [1981a] and Jaffe and Ferrara [1984]). The approach of Kohberger et al. [1978] is less straightforward. They define an aggregate weighted measure of the variations about a nominal reference state trajectory that would result from variations in the model parameter values; a sample set of realizations of the variance measure can then be generated, and finally this sample (as the dependent variable set) can be regressed on a second-order function of the sample parameter values. Further analysis of the properties of the resulting regression relationship yields the required ranking of the contributions of the individual parameters to the variance measure.

It cannot, of course, be expected that any general pattern of conclusions on the significance of the different sources of uncertainty should have emerged. Case study results tend inevitably to be problem and model specific. For example, parameter uncertainty was found to be much more significant than initial state or input disturbance uncertainty in the study of Scavia et al. [1981a]. Yet in contrast, Somlyody's [1983] analysis of a one-dimensional model for the seiche behavior of Lake Balaton indicated that uncertainty in the wind direction (a measured input disturbance) would be considerably more important than uncertainty in the model parameters such as the bottom friction coefficient. Notable too is the observation from the analysis of Gardner et al. [1980a] of the predatorprey models of $O^{\prime}$ Neill et al. [1980] that the effects of parameter uncertainty dominate over the effects of "modeling error," assumed in this case to be due to an erroneous model structure. This observation does, however, require clarification, for the results refer to errors arising from alternative model structures relative to the predictions from a reference, and assumed "true," model structure. Within the scheme of Figure 1 the (a posteriori) residual errors and identified model structure would be inextricably reflected in the a posteriori parameter estimation error variance matrix $\left(P^{p}\right)$, and also possibly $P^{c}$. It is therefore perhaps a weakness of this scheme that such distinctions cannot be made, although the need for, and capacity to calculate, them is not immediately obvious.

Of much more immediate concern is the result that the two different methods of ranking the sources of uncertainty, according to relative sensitivity coefficients (first-order error analysis) or according to correlation coefficients (from Monte Carlo simulation), give significantly different conclusions [Gardner et al., 1980a; Halfon, 1984]. And the important implication of a wrong ranking of the significance of the various sources of uncertainty is that the wrong experiment will be designed to reduce the uncertainty in any subsequent predictions.

7.4.5. Experimental design and the reduction of uncertainty. From the (original) experimental data, identification has yielded knowledge of the (internal) description of the system's past behavior and knowledge of the uncertainty that circumscribes the interpretation of this behavior. We have examined the propagation of that uncertainty forward into the prediction of future behavior, distinguished the most important sources of uncertainty giving rise to poor predictions, and hence can now ask the question of what new experiments can be designed in order to reduce the most critical uncertainties. Thus the review has turned a full circle. This is, once again, and as already discussed in section 2, problem (P4). But given now the greater body of prior knowledge of the system's behavior and uncertainties, the problem of experimental design can be considered in less primitive terms than before. In particular, if the relationship between the state variables and the measured output response variables is assumed to be of the form of ( $32 b)$ (for the class II model structure), i.e.,

$$
\mathbf{y}\left(t_{j}\right)=\mathbf{h}\left\{\mathbf{x}, \boldsymbol{\alpha} ; t_{j}\right\}+\boldsymbol{\eta}\left(t_{j}\right)
$$

the problem of experimental design can be formulated within the conceptual framework of control theory (more precisely, as a filtering problem; Moore [1973], Moore et al. [1976], Lettenmaier and Burges [1977], and Canale et al. [1980]). A more sophisticated experimental design problem can therefore be postulated, i.e., one that is conditioned upon a knowledge of uncertainty:

Given a model, knowledge of the internal description of the system's behavior $\{\mathbf{x}, \boldsymbol{\alpha}\}$, and its associated uncertainty, how will information about the external description of the system $\{\mathbf{u}, \mathbf{y}\}$ and its associated uncertainty, alter the knowledge of $\mathbf{x}$ and $\boldsymbol{\alpha}$ ?

In simple terms, several authors have remarked that a higher frequency of sampling (of $\mathbf{u}$ and $\mathbf{y}$ ) should be allocated to (predicted) periods of rapid change in the state variables (or equivalently of maximum biological activity) because these are periods of maximum prediction error variances [Gardner et al., 1980a; Scavia et al., 1981a; Lettenmaier and Richey, 1979; Jorgensen et al., 1981]. This is clearly similar to the comments on sensitivity coefficients, identifiability, and experimental design in section 2, in particular, the discussion of Figure 2, 
but it differs in respect of the reference to prediction uncertainty.

In more advanced terms, the above problem of experimental design can be cast as a problem of constrained optimization, of minimizing the cost of the measurement strategy subject to constraints on the leading diagonal of the state estimation error covariance matrix, i.e., $P^{s}\left(t_{j}\right)$ in (37a) [Canale et al., 1980]. For example, Figure 14 shows the propagation of error variance about nominal (predicted) state $\langle x\rangle$ and parameter $\langle\alpha\rangle$ trajectories for phosphate concentration and a phytoplankton growth rate constant in one of the spatial segments of a model for Saginaw Bay, Lake Huron [Canale et al., $1980]$. In the event that no further observations are made of the system, the uncertainty in the state prediction propagates with increasing magnitude, while the parameter uncertainty remains unchanged. In other words, this is simply the solution of (37), i.e., use of the prediction step alone of the filtering algorithm presented as (12) in section 4 . However, were a new, "near-optimal" monitoring program to be implemented, both error variances would be progressively reduced. In this case, the assumption is that future observations of the output response (y) of the system would be available and that these observations would be uncertain, with an error variance of say, $R$, associated with $\eta$ in (41). Without necessarily specifying what the values of $\mathbf{y}$ would be, this enables use of both the prediction and correction steps of the filter of (12), the reduction in the error variance propagation being brought about specifically by $(12 d)$.

\subsection{Commentary}

Unlike the topic of identification, which suffers from a weakness in the availability of appropriate methods, the analysis of prediction error propagation needs perhaps not so much new methods and approaches as new questions about what the analyst wishes to achieve in making predictions.

(a)

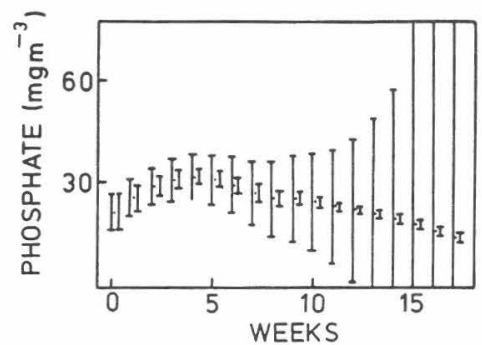

(b)

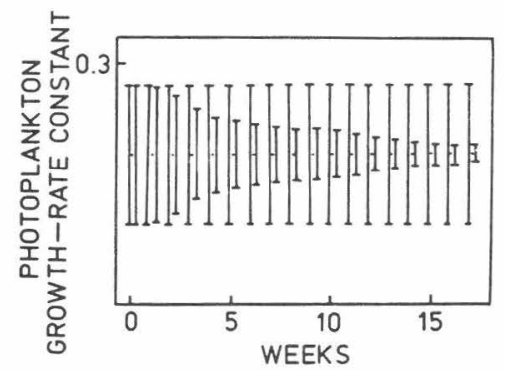

Fig. 14. Analysis of error propagation in a case study of monitoring program design for Saginaw Bay, Lake Huron [after Canale et al., 1980]. (a) Phosphate concentration. (b) A phytoplankton growth rate constant. Dots represent the predicted state $x$ or parameter $\alpha$, and the first bar symbol of each such pair represents the one standard deviation bounds of the errors for a "zero" measurement strategy, while the second bar refers to a "full" measurement strategy.
There are certainly good reasons for continuing to explore the limitations of first-order error analysis vis a vis the use of Monte Carlo simulation, for erroneous conclusions can be drawn, in particular, about how to set about reducing prediction uncertainty. But for most practical purposes these methods are well tested and do, by and large, deliver the answers sought to the questions asked. Such satisfactory preformance cannot, for comparison, be attributed to the problems discussed throughout sections 3-6. If anything, the straightforward analysis of prediction error propagation has received less attention in the past 2 or 3 years, suggesting possibly a shift of interest toward issues of management and decision making under uncertainty (as discussed in section 8).

Perhaps surprisingly the choice of types of probability density function characterizing the sources of uncertainty has not been extensively discussed. One exception is the analysis of a stream ecosystem model by $O^{\prime} N$ eill et al. [1982a], who have concluded that their model yielded expected values and coefficients of variation that were insensitive to the choice among uniform, triangular, and Gaussian distributions (by far the most commonly used statistical assumptions). This implies neither a lack of statistical rigor nor a lack of curiosity about extreme-value events. Rather it reflects a lack of sufficient field data, especially for variables of a biological or biochemical character, by which to justify a more refined choice of density function.

Most of the evidence suggests that the current models of water quality, in particular, the larger models, are easily capable of generating predictions to which little confidence would be attached. Yet there have been few case studies of the truly larger scale models, and the challenge that they represent (as O'Neill and Gardner [1979] have described it) has not been adequately met. In this respect, much remains to be done.

On balance, it has to be accepted that a first-order error analysis will always be suspect in its accuracy because of the inherent approximations of linearization. It is similarly not as complete in its analysis as Monte Carlo simulation, but it may well be less cumbersome in its mechanization of the effects of correlated errors and in being able to preserve the relationships between prediction error and the various sources of uncertainty (which are particularly evident from the structures of (36) and (37)). It must also be an advantage of first-order error analysis (as yet not fully exploited) that it can be interpreted in the context of filtering theory and hence viewed more naturally in the Bayesian terms of this review. This conceptual setting of first-order error analysis may indeed prove to be more fruitful in stimulating new questions for the analysis of prediction error propagation. It may then be that Monte Carlo simulation is the more appropriate method for addressing the numerical solution of these new problems.

However, before turning to a possible agenda of problems for the future, let us close this discussion of prediction error propagation - of predictability itself - with an apparent paradox. In a stochastic model, whose parameters vary randomly with time, the faster the rate of random parametric variation, the less is the variability of the predicted state of the system [Kremer, 1983] (see also Tiwari [1979] and related discussion by Finney et al. [1982]). Consider now a deterministic nonlinear model, with no stochastic elements, that predicts essentially chaotic behavior [May, 1976]. Could it be, if we believe our common experience that reality is orderly and somehow predictable, that out of randomness comes forth order (see also O'Neill et al. [1982b])? 


\section{Further Studies}

It is perhaps a foolhardy reviewer of the subject of uncertainty who would speculate about the directions of future research. But let us begin by reiterating the main trends of the discussion from both section 2 and sections 3-7.

The objective throughout the whole of this review has been to steer a middle course between opposing approaches to modeling that are either overly complicated or overly simplified, albeit with a distinct tendency to err on the side of simplicity. It would be naive to imagine that there is a best approach to the development of models for identifying and predicting the behavior of environmental systems, any more than there is a best model for a given problem. While it is always possible to construct a model that includes every detail of conceivable relevance, what we have loosely labeled a class I model, such a model is not an effective prescription for the exhaustive interpretation of field data. And while it is equally possible to interpret time-series field data via a simple input/ output model (a class III model), this is insufficient if it is not coupled with a procedure for the assessment and revision of prior theories about the phenomena governing process behavior. So there has been some movement toward a position in which these two polarized approaches can be seen as complementary: the one representing more the assembling of an archive of hypotheses (class I), the other representing a vehicle for the interpretation of field data (class III).

Any discussion of the (scientific) merits of a preferred approach to modeling by reference to observed behavior tends ultimately to be deflected into a sterile discussion of goodness of fit to the data. It is thus difficult to expose the limitations of the various approaches unless, that is, the debate is cast in terms of the question of prediction. It is prediction too that is of direct relevance to management and decision making. In fact, there is a dilemma [Beck, 1981b, 1983]. Intuition would have led us to suppose that the comprehensive model would be superior in its "predictive power," such superiority resting upon the assertion that the simpler models (identified from an inevitably restricted sample of past observations) would be unable to predict the future under conditions substantially different from those of the past. But is this superiority justified? For we have now seen that this assertion is crucially suspect in overlooking the problem of identifiability, i.e., the ambiguities of representing past behavior, and the propagation of (possibly gross) uncertainties in predicting future behavior. The dilemma is that the simpler model, reflecting only the observed behavior of the past, and being presumably well identified, will predict an "incorrect" future and, worse still, suggest possibly substantial confidence in that prediction. The more complex model, although it may be capable of predicting a "correct" future, will be reliant upon apparently redundant or ambiguous hypotheses for that prediction, which should not therefore be accorded much confidence. Moreover, such models may be capable of generating equally probable, but quite contradictory predictions.

So again, the middle course may have something to contribute, and it does seem to echo Botkin's [1977] conjecture that, "if small is beautiful, and big is ambiguous, then middle-sized is meaningful." It too, however, is by no means without limitations. Suppose we take the form of our intermediate class II model structure. It reflects a position on the identification of models that favors simplicity in the prior hypotheses, makes some relatively direct reference to the physical, chemical, and biological phenomena believed to govern observed behavior, and attempts to interpret the field data in like terms. Such a strategy will always pose the substantial difficulty (as discussed in section 4) of having to choose or to generate additional hypotheses to be included in a demonstrably inadequate model structure. This is arguably neither better nor worse than the problem of starting with a comprehensive model and then handling the difficulty of identifying those redundant hypotheses that are to be excluded from the model structure (assuming it were first possible to demonstrate the inadequacy of the structure, which is debatable).

Not surprisingly, then, there are many unresolved issues for the further development of the subjects of uncertainty, identification, and prediction in water quality modeling. The key questions to be addressed in this section are as follows.

1. Are the basic problems of model identification ones primarily of inadequate method or of inadequate forms of data?

2. What opportunities are there for the development of improved, novel methods of model structure identification, in particular, with regard to exposing the failure of inadequate, constituent model hypotheses?

3. How can an archive of prior hypotheses be appropriately engaged in inferring the form of an improved model structure from diagnosis of the failure of an inadequate structure? Moreover, in what form should the knowledge of the archive be most usefully represented?

4. What does a lack of identifiability imply for the distortion of a model structure, and what are the consequences of a distorted model structure in terms of generating predictions?

5. Given uncertainty, how can one speculate about the prediction of a "radically different" future?

6. What, in the end, does all this mean for decision making under uncertainty?

\subsection{The Nature of the Data}

When all the sophistication of the algorithms for identification is stripped away, the inescapable precondition for their successful application is that the dynamic changes in the input variables (u) are almost self-evidently related to corresponding changes in the output response variables (y). Consider, as an illustration of the ideal, the classic tracer experiment for determining the advective transport and dispersion of a pollutant along a river. There can be little doubt that the typical pattern of the downstream output response concentration (of Figure $15 a$ ) is unambiguously related (in some way) to the upstream input pulse of tracer. Now consider, by way of contrast, the fast (high frequency) output response of an algal bloom in a lake, to which no obviously impulselike change in input solar radiation or nutrient loading pattern corresponds (Figure $15 b)$. What we see in terms of the external description of the system $\{\mathbf{u}, \mathbf{y}\}$ in this case is "apparently insignificant input perturbation: significant output perturbation." It might conversely occur that the in situ field data exhibit "apparently significant input perturbation: insignificant output perturbation," a situation commonly found in the performance of biological wastewater treatment processes (Figure 15c; see also Beck [1986]).

Either of the latter two cases is precisely what one would wish to avoid for the purposes of model identification; they reflect strongly nonlinear input/output relationships. Most methods of identification, suitable for the form of external description of Figure 15a, assume a priori a simple, linear 


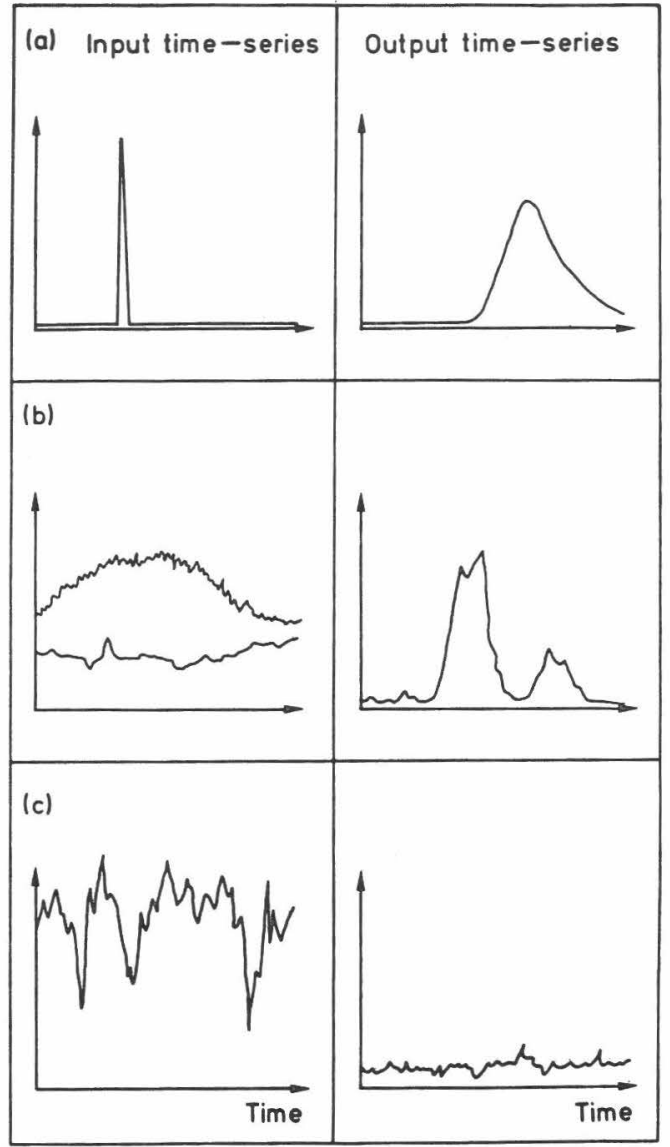

Fig. 15. Stylized examples of input and output field data patterns for $(a)$ a dye tracer experiment in a river, $(b)$ the occurrence of an algal bloom in a lake, and $(c)$ a secondary biological wastewater treatment process.

model structure, and from there can proceed to the identification of weak forms of nonlinearity (see section 4). Even to begin to interpret the external descriptions of Figures $15 b$ and $15 c$, it is necessary a priori to postulate virtually the correct form of (strong) nonlinearity, and this is notoriously difficult for, say, the population dynamics of algae (M. B. Beck and B. A. Finney, Operational water quality management: A case study of the Bedford Ouse River system, submitted to Water Resources Research, 1986).

The difficulties of identifying environmental systems are thus not merely that the data are uncertain, but that they are derived from poor approximations of the classical experiments of laboratory science.

\subsection{Failure}

In section 4 the idea of model structure was defined as a complex amalgam of constituent hypotheses. In order to understand what was meant by the selection and evaluation of that structure we introduced a conceptual analogy between a model structure and a physical engineering structure. The general problem of model structure identification, irrespective of the method used for its solution, is the need to expose unambiguously the failure not of the model as a whole, but of the constituent model hypotheses. The failure of a hypothesis can be likened to the failure of a component member of a physical structure subjected to various loads, and in other ways this same notion can be equated with Popper's view of the scientific method.

The use of recursive estimation algorithms is one specific means of solving the problem of model structure identification, indeed one that has had much to do with the definition of the problem. In section 4, temporal variation of the estimated values of the model parameters was taken to be indicative of the failure of a constituent hypothesis. Thus in the model structure or "graph" of Figure $5 b$, the nodes represent the model's state variables $(\mathbf{x})$ and the branches (structural members) the model parameters $(\alpha)$. The external loads placed on the (model/engineering) structure may be assumed to be equivalent to the errors of mismatch between observed and estimated behavior. The "distortion" necessary for the model structure to be matched with the structure of the dynamics underlying the observations is reflected in the deflections of the recursive parameter estimation trajectories (such as those of Figure $5 c$ ). The capacity of a structural member (hypothesis) to resist deformation, i.e., its mechanical properties, corresponds in some way to the confidence (uncertainty) attached to that constituent hypothesis (as parameterized through $\alpha$ ). And last, the more rigidly (confidently) the model structure is stated, the more easily demonstrable ought to be its failure.

The questions that one would like to have answered are the following: has a failure of a constituent hypothesis occurred, what are the relative weaknesses/strengths of the individual hypotheses, and what is the connection between the failed hypotheses and the mismatch between the model and the observations?

However, experience shows that clear answers to these questions are difficult to obtain because (1) the performance of the extended Kalman filter (EKF) is not as robust as would be desirable and, inter alia, is heavily compromised by the need to make more or less arbitrary assumptions about the sources of uncertainty affecting the identification problem, (2) the instrumental variable algorithm, while it overcomes these limitations of the EKF, does not (as usually implemented) make direct reference to the physical, chemical, and biological origins of the constituent model hypotheses, and (3) it is difficult to absorb and interpret the wealth of diagnostic evidence deriving from the test of the model structure.

This third point will be dealt with in a later section. More immediate, and more compelling, is the idea that the advantages of the IV and EKF algorithms should be combined, and their disadvantages eliminated.

It has to be significant then that with one and the same minor modification (use of an innovations process representation) both can be shown to solve the dual of the primal estimation problem for which each was originally intended, i.e., (1) accurate parameter estimation with the EKF [ Ljung, 1979], which as we have said in section 4, is an algorithm originally intended for the purposes of state estimation, and (2) state estimation with the IV [Young, 1979], an algorithm clearly developed initially for the purposes of parameter estimation.

It is impossible to resist the temptation to observe that such simultaneity of publication date, similarity of authors' names, and symmetry of algorithmic structure must be auspicious! Indeed they are. The ramifications of these developments are several and go well beyond the scope even of this review. They are also complex, convoluted, and although directed ultimately at simplified algorithms for model structure identif- 
cation, difficult to summarize in the straightforward, qualitative terms that follow (other preliminary comments are given elsewhere; Beck $[1985 c, d]$ ).

First, and of most immediate significance, Ljung's modified form of EKF conducts the estimation of the model parameters as though there were perfect knowledge (i.e., no uncertainty) of the state variables, which is notably what the IV does in any case. This obviates the need to make some of the notoriously arbitrary assumptions about the sources of uncertainty influencing the identification problem, which have so limited the ease of implementing the ordinary EKF. It ought also to lend robustness to the performance of EKF-like algorithms.

Second, the mechanism by which the above is achieved involves estimation of the filter gain matrix elements (for which purposes the assumption of an innovations process representation is necessary). Adaptation of the model's stateparameter estimates as the data are processed sequentially, which lies at the heart of our interpretation of model structure identification, becomes thus less dependent on arbitrary prior assumptions and more dependent on the posterior properties of the actual errors of mismatch between the model and the data.

Third, and rather more speculative, if the primal problem has been stated as estimation of the model parameters given perfect knowledge of the state, the dual problem would be that of estimation of the states given perfect knowledge of the parameters. This pair of dual problems corresponds with the quality of mesh and nodal descriptions of networks such as that of Figure $5 b$. We know that graphs, networks, and connectivity are intimately related to the analysis of structural identifiability [e.g., Cobelli et al., 1979]. We know too that structural mechanics is based upon graph theory and network representations [Spillers, 1972] and still further that dual mathematical programs play an important role in the plastic limit analysis of loaded engineering structures [Munro and Smith, 1972]. If therefore the problem of model structure identification has an analogy in the problem of plastic limit analysis, one must ask the potentially very fruitful question, Do the existing solutions to plastic limit analysis suggest future analog solutions to model structure identification?

Last, and in a more philosophical vein, let us note that assuming perfect knowledge of the parameters is the limiting case of making those bold, confident hypotheses that Popper has argued are essential to the application of the scientific method [Popper, 1968]. In this respect, the trend of what has been said in this section would appear to be in the right direction and, moreover, meets many of the objections raised in the commentary to section 4 on model structure identification. But making bold, perfectly confident hypotheses (i.e., in our terms, deterministic, constant parameters) and then seeking to detect the failure of these hypotheses via the temporal variability of the estimated model parameter values is an apparent paradox. So how might such a test be implemented? This is extremely difficult to answer, but one attractive possibility would be to represent the "bold, confident hypotheses" as a deterministic, nonlinear, continuous-time reference trajectory model (such as (7) of section 4) and to interpret the failure of these hypotheses via the estimated parameters of a corresponding discrete-time small-perturbations model (such as (10) of section 4). The resulting estimation problem would also have the desirable properties of referring to algebraic equations that are linear in the parameters and would therefore be an ideal response to the remarks made in the commentary of section 5 on estimation.

All of these points, however, are highly speculative, not substantiated, and like the bold, confident hypotheses, may themselves turn out to be demonstrably wrong. But they are still worth mentioning because they offer the possibility of escape routes from the narrow methodological confines into which (in this reviewer's opinion) the subject of identification and parameter estimation has fallen in recent years.

\subsection{Inference}

The essential purpose of modeling is the need to understand the behavior of a system either in order to give a satisfactory scientific explanation of that behavior or to give advice for the guidance of decision making. There are three broadly different types of knowledge that enter into the acquisition of this understanding.

1. Observed knowledge of the external description of the system, i.e., the experimental observations of the system's inputs and outputs $\{\mathbf{u}, \mathbf{y}\}$.

2. Theoretical knowledge of the internal description of the system, i.e., the constituent hypotheses cast in terms of the model's states and parameters $\{\mathbf{x}, \boldsymbol{\alpha}\}$ (and their relationships with the inputs and outputs).

3. Knowledge of a diagnostic, interpretative character about the mismatch between the system and the model, e.g., the failure of constituent hypotheses and the identification of anomalous events.

If we were content merely to order and classify the observed facts, without further interpretation, then we would need no more than the first type of knowledge, and almost all of this review would be irrelevant. However, this is not the case, and it has become the tradition (over many years) to seek to represent knowledge in the abstract form of mathematical relationships and in as complete (and elegant) a form as possible, i.e., the class I model as defined in the introduction to the review. With the advent of the digital computer this archiving of hypotheses, of knowledge of a theoretical type, has been practically removed from virtually all constraints other than the creativity of the mathematical analyst to express equations for those (observed) phenomena he believes he understands. This review has had little to do with that process. In fact where the review has discussed model development, in the context of identification, it has concerned itself with the development of theories strictly by reference to the in situ field data, and using the less complete (less elegant) class II and III models as vehicles for that process. As such, all of this review (of system identification) and now the comments of the preceding discussion of failure have been about increasing knowledge of the third type, that is, knowledge of a diagnostic character about why a model fails to describe observed behavior.

The problem of inference (as intended here) is therefore the problem of reasoning about the possible form of an improved posterior model structure given diagnostic knowledge of the failure of a prior model structure. This reasoning is one of synthesis because by a sifting and piecing together of the diagnostic evidence (like the forensic science called for in section 5 ), it must proceed to a "good hunch" as to why the model failed and how it might be improved. It is also inductive and, thus being akin to the creative process of scientific discovery, is not entirely capable of being reduced to some logical algorithmic form [Beck, 1985d] and should not be so reduced. 
So what can be said of tentative solutions to this problem of inference? Our response involves examination of the nature of the inferential reasoning process, which brings into question the nature of knowledge representation, which in turn introduces both a challenge to conventional wisdom on model building, and a quite different view of the problems of uncertainty, identifiability, and predictability.

\subsection{Knowledge Representation}

Consider the case study of wind-induced resuspension of sediment material in a shallow lake that was discussed in section 4. On identification it was found that the prior model structure failed to characterize observed behavior over the last 40 days or so of the experimental record, in that the model persistently overestimated the observed suspended solids concentration (Figure 7). This anomaly requires explanation. Several hypotheses (at least three) could be plausible candidates, one of which concerns the possible effects of wind fetch length. The actual reasoning that then took place-insofar as it can be recalled-was of the following form: "if" wind fetch length increases (decreases), "then" water surface shear stresses increase (decrease), "and" sediment shear stresses increase (decrease), "and" more (fewer) sediment particles are resuspended.

There is nothing novel about this form of reasoning, as any developer of a model will know. Indeed, it is so commonplace that it is difficult to notice what might be its importance in the present discussion.

First, the central problem of inference is the incompatibility of the various forms of knowledge used in its solution. For instance, taking another aspect of the Balaton example [Beck, 1985d]: how can diagnostic knowledge concerning correlated variations between observed temperature and an estimated parameter in an algebraic, input/output (class III) model be matched with theoretical knowledge of particle motion in a partial-differential equation representation (class I model) in order to change the structure of an ordinary-differential equation (class II) model? What the above example of inferential reasoning suggests is that it is the imprecise, linguistic knowledge at the basis of our mental models of how systems behave that may be the most useful common denominator of the various types of knowledge and knowledge representation.

Second, if this is the case, then it is not the precise partialdifferential equation representation of particle motion that is crucial to this kind of inferential process, but rather the more primitive chains of less precise, more macroscopic logical reasoning.

Third, there would be little point in concerning ourselves with this qualitative form of reasoning if no additional assistance could be given to what we already practice with great facility. And this is where the much talked-of recent developments in the expert systems of artificial intelligence may have a significant role to play [Duda and Shortliffe, 1983; Forsyth, 1984].

Fourth, since we can therefore represent and manipulate knowledge in the qualitative, imprecise, linguistic terms of, for example, fuzzy logic [Tong, 1978; Bosserman and Ragade, 1982; Jowitt, 1984; Camara et al., 1985], what are the implications of this for the "classical" approach to uncertainty, identification, and prediction upon which the whole of this review has been built?

The implications, if not profound, are undoubtedly provocative. Consider the following conjecture. If the systems whose behaviour we attempt to describe are inherently imprecise, and if the observations that can be made of such systems are also imprecise, it is illogical to entertain algebraic or differential forms of equations as candidate descriptions of the system. This conjecture has merit, and a very preliminary reaction to expert systems would be that they seem to work best when the system's dynamics are highly nonlinear, as observed for instance, in the earlier discussion of Figures $15 \mathrm{~b}$ and $15 c$; a theory is in its initial phases of development, e.g., as a verbal, conceptual model; crude order must be imposed on a confused and conflicting welter of experimental observations; and decision making must be conducted in a setting where a pragmatic, universal shortcut to interdisciplinary communication is a priority (a matter to which we shall return below).

But will our observations always remain imprecise, and will our theories never develop beyond their initial stages?

Consider further the following. Suppose the opinion of an expert biologist or geochemist is sought for the description of the behavior of a given lake system. In effect, what is being asked for (and what will be given) is an interpretation of existing theories and casual experimental observation. The logical rule that results - most naturally in the form of a cause-effect statement - is an amalgam of theoretical and observed knowledge. This process is now explicitly subjective but then so too, implicitly (upon careful reflection), is the classical approach to model building. The distinctive roles of theory and observation have become blurred. It is not clear what has happened to the role of identification and diagnosis. Nor is it clear whether any principles of scientific method do or should govern this process (perhaps it has become "anarchistic" in Feyerabend's [1975] terms).

The instinctive, long-term inclination of the scientist is to give his theories as much precision, rigidity, and independence as possible. Insofar as it is possible, we wish to set out theories apart from subjective interpretation. Or do we? Can we, ultimately, accept imprecision (as opposed to uncertainty) as fundamental to nature?

\subsection{Questions for Prediction}

It has been said that the variance (uncertainty) properties of the posterior parameter estimation errors can be thought of as a synopsis of the results of forcing a model structure to fit the data. In terms of the analogy of a physical engineering structure, such "forcing" may cause a distortion of the model structure. And in much the same way as we have already discussed the possibility of a model structure in speculating about the interpretation of past behavior, so this metaphor can be exploited in order to study the implications of distorted structures molded by the fitting process to possibly quite different shapes.

Suppose that the model under study has been fitted to a set of data, that the model structure suffers from problems of identifiability, and that many combinations of the parameter estimates give "acceptably good" fits to the data, two of which might correspond with the pair of distorted structures in Figure 16. Unwittingly either version of the fitted model structure might be used for the purposes of predicting the longterm future behavior of the system. For instance, Figure $17 a$ shows two sets of predictions that could have been generated by the two equally possible structures of Figure 16. In fact these results refer to the behavior of a phytoplankton population in a model of Lake Ontario [Halfon, 1979], the differ- 


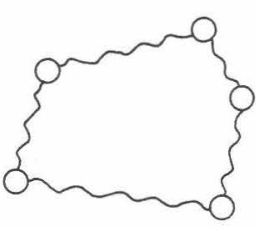

(a)

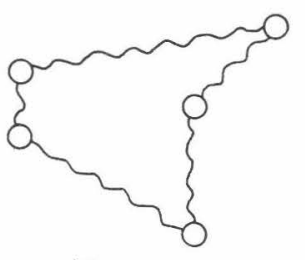

(b)
Fig. 16. Conceptual analogs of two equally possible versions of a model structure resulting from the problem of model identifiability.

ence in initial values deriving from the different "equilibrium" annual cycles established after a transient from the present state (M. B. Beck and E. Halfon, manuscript in preparation, 1986).

What would one conclude from such predictions? Whatever the temptation, no conclusions should be drawn except, of course, to doubt the value of purely deterministic predictions and to raise again a question mark over the predictive powers of comprehensive models. One might have expected the conclusion that there may be so much uncertainty attached to these predictions that literally anything could happen in the future. This too would be inappropriate. In Figure $17 b$ the bounds for the alternative predictions lying within one standard deviation of their respective mean (i.e., deterministic) trajectories are indicated. All errors have been assumed to be normally distributed, only errors arising from the model parameter estimates have been accounted for, and in fact the results shown have been computed from the algorithm of (37) in section 7. There are times when the predictions do not differ significantly, i.e., they are "indistinct," times when their means are seemingly different but there is sufficient uncertainty to render these predictions "ambiguous," and many times when the nominal trajectories are substantially different and with sufficiently little error that they are confidently "contradictory."

Such reasoning may be spurious, and certainly these results are based on the strong assumption, among others, that identical variance-covariance structures hold for different nominal sets of parameter estimates. Consider, however, that for the problems of surface water acidification there is every possibility of needing to make decisions in the face of competing (and conflicting) hydrological and hydrochemical theories. Given all the existing uncertainties, the questions that we would like to see opened up by this type of analysis are (1) is it possible to distinguish any significant difference between the consequences of competing theories that give equally plausible explanations of past behavior, (2) is it possible to design a crucial experiment that will distinguish among competing theories, (3) how confident do we have to be of our models in order to distinguish significant differences among the responses of the system to alternative input scenarios, (4) if the system's predicted behavior is not sensitive to the unidentified constituent hypotheses of a part of the model and is only sensitive to their collective effects, is it really necessary to concern ourselves with a lack of model identifiability [see also McLaughlin, 1985], and (5) how would it be apparent that the postulate of this question (question 4) is valid?

The problem of identifiability, which throughout this review has been treated as highly undesirable, may not therefore have material consequences for the problem of prediction (subject to the technicality of question 5). In fact a lack of identifiability, instead of being undesirable, may even be of benefit in trying to answer certain questions, as we now discuss.

\subsection{The Future Under Substantially Changed Conditions}

Hitherto, the questions of prediction examined here have all been based on a conventional problem statement.

Question 1 (Q1). Given the model structure, its parameter estimates, the future input disturbances (and, of course, specifications of the relevant sources of uncertainty), what future behavior is generated?

Suppose, however, that this question is turned on its head, so that (incorporating some of the ideas discussed in section 3) we have the following.

Question 2 (Q2). Given definitions of acceptable (B) and unacceptable $(\bar{B})$ future behaviors, to what constituents of the model structure and assumed future inputs are these behaviors most/least sensitive?

Or the question can be put in a much more interesting fashion.

Question 3 (Q3). Given definitions of essentially similar $(S)$ and radically different $(\bar{S})$ future behaviors, determine answers similar to those of (Q2).
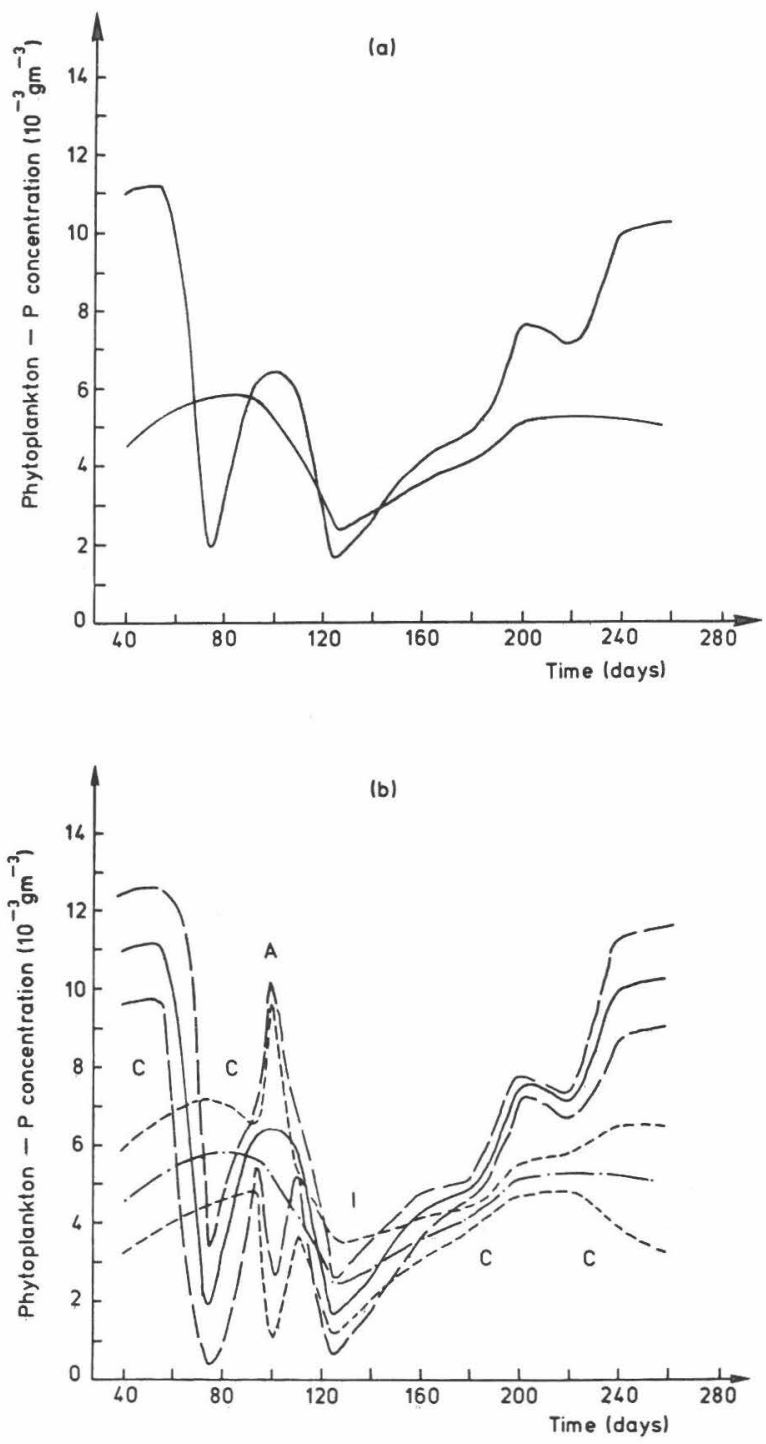

Fig. 17. Predictions derived from two equally possible combinations of model parameter values: $(a)$ deterministic predictions and (b) deterministic predictions and plus or minus one standard deviation bounds (dashed lines) on the errors associated with each stateprediction $x$. A, ambiguous; C, contradictory; and I, indistinct. 
Provided therefore that the analyst can define what he understands by the future "under substantially changed conditions" (and it is no bad thing that he should be forced to do this), he has thus a means of locating its causal mechanisms. And if there are constituent hypotheses in the model that support this radically different future behavior, then in principle they ought not to be identifiable against the record of past behavior. In other words, we have a reversed form of logic in which the implications of a statement about the future can be tested against the record of the past.

\subsection{Decision Making Under Uncertainty}

There has always been decision making under uncertainty, and there has been quantitative analysis ostensibly in support of it for at least 20 years [Loucks and Lynn, 1966]. Present trends suggest that in the area of water quality management there is a growing momentum of interest in a variety of methodological alternatives for incorporating uncertainty into the analysis of decision making. This interest can be separated broadly into five classes.

1. The use of Monte Carlo simulation, either simply to calculate the distributions (variability) of water quality characteristics that would result from alternative management scenarios [Whitehead and Young, 1979; O'Neill et al., 1982c, 1983] or to assess the risk of violating a prescribed standard [Fontaine, 1984; Chapra and Reckhow, 1983].

2. The solution of problems of constrained optimization under uncertainty [Lohani and Thanh, 1979; Lohani and Saleemi, 1982; Fisher, 1983; Herbay et al., 1983; Somlyody and Wets, 1985; Fontaine and Lesht, 1986].

3. Extension of problem 2 to the notion of fuzzy programming in order to derive "satisficing," as opposed to optimizing, solutions [Chuang and Munro, 1983].

4. The more general application of fuzzy logic (and expert systems), either as an operational means of gauging the performance of a pollution control authority [Jowitt and Lumbers, 1982] or as a means of system simulation and prediction in an interdisciplinary, decision-making environment [Camara et al., 1985; Fedra, 1985].

5. Use of the ideas of decision analysis along the lines suggested by Chapra and Reckhow [1983] for the control of polychlorinated biphenyls (PCB) discharges to the Great Lakes.

This looks promising, but the real question is whether any quantitative analysis of uncertainty has been, or is being, used to assist decision making in practice.

As a matter of actual policy, current practice in the United Kingdom with regard to the control of river pollution is to analyze the impact of polluting discharges in probabilistic terms in order to set probabilistic standards to be satisfied by those discharges [Warn and Brew, 1980]. Considerations of uncertainty are therefore integral to the monitoring of compliance with the given standards [Warn and Matthews, 1984]. As a matter of proposed policy, the U.S. Water Pollution Control Federation recommended in 1981 that the following would be desirable.

Analyzing the wasteloads for water quality-limited streams using verified mathematical models that are calibrated with local information.

Consider setting aside some of the stream's capacity as a reserve for future discharges and as a hedge against errors or inaccuracies in the predictions made with the model.

[Water Pollution Control Federation, 1981].
As a matter of independent comment on this review, many of its elements are referenced elsewhere in a document commissioned by the Dutch government on "Handling uncertainty in environmental impact assessment," where they appear (quite appropriately) as an appendix of brief details [Ministry of Public Housing, Physical Planning, and Environmental Protection, 1985].

And last, as a matter for the reader to consider for himself, Somlyody and van Straten [1986] have described recently a uniquely comprehensive study of managing eutrophication in Lake Balaton, Hungary, in which the issue of uncertainty permeated essentially every aspect of the supporting mathematical analysis. In their book, the political "background to a decision" is discussed at length [Lang, 1986]. Given this evidence, the questions are, Did the analysis influence the decision; if so, in what way; and more generally, is it really necessary to spend a lot of time to establish the relevance of scientific analysis to management?

\section{CONCLUSIONS}

The purpose of this review has been to assess the role of uncertainty in the development of mathematical models for the interpretation and explanation of past observed behavior and for the prediction of future behavior. It has not been the intention to review the relevance of these subjects to problems of decision making under uncertainty.

The contributions of over a decade of research into the analysis of uncertainty in water quality modeling have been many and varied. Perhaps the most innovative has been the HSY algorithm of section 3, a conceptually simple means of generating preliminary hypotheses about the behavior of a system under conditions of sparse field data and gross uncertainty in the prior theoretical knowledge of the system's behavior. As such, this problem has been long standing, and it is surprising that it was not properly recognized and addressed until relatively recently.

If adequate numbers of (time series) field data are available and the prior theoretical knowledge is less uncertain, there emerges a more refined problem of model structure identification (section 4). The primary advance of the past decade has been one of improved problem definition. In other words, it is now more clear what sort of questions should be asked of this type of analysis and on what basis they might be answered, i.e., by seeking to expose the failure of constituent model hypotheses and then attempting to infer the form of an improved model structure from diagnosis of the failed prior structure. This advance has been gained largely through the application of recursive methods of estimation, even though these methods may not eventually be the best means of solving the problems of model structure identification, and can certainly be shown to have several limitations.

Given the identified model structure, model calibration or parameter estimation would have seemed a straightforward problem to solve with no shortage of available methods of solution. On the whole, this has not proved to be the case. Most attempts at solving this problem have not been entirely successful because of a :ack of model identifiability. In addition, little attention has been paid to evaluation of the residual uncertainty associated with the estimated model parameter values (section 5). The conclusion of this review is that a lack of model identifiability is unlikely to be overcome in the near future by improvements in the associated methods of numeri- 
cal optimization. The profit to be derived from this failure is that model identification (of which parameter estimation is merely a part) should be more usefully viewed as a kind of forensic science, a painstaking piecing together and sifting of all the evidence obtained from a variety of lines of investigation, with the objective of providing a plausible and rigorous explanation of why the system behaved as observed.

The case of prediction under (explicit) uncertainty is rather different from that of identification under uncertainty. The initial set of questions has been well defined and adequately answered (section 7). We now know that the larger models of water quality may give highly uncertain predictions of future behavior, and that caution should be exercised in the use of a first-order error analysis (as could be expected), especially with regard to ranking the importance of the various contributing sources of uncertainty. For prediction, it is not so much new methods of analysis that are required in the future, but rather a more wide-ranging set of questions to be answered.

In the long term, however, whence has the subject of uncertainty in water quality modeling come, and whither is it destined? We might speculate that just prior to the seminal studies of Streeter and Phelps [1925] there would presumably have existed some simple, imprecise, verbal models of the behavior of water quality in river systems. There were no electronic computers. By the end of the 1970 's, under the liberating influence of powerful mainframe computers, there were immensely complex, seemingly arbitrarily precise, and predominantly deterministic models of water quality [Chen and Smith, 1979]. There was too a nascent awareness of uncertainty [O'Neill and Gardner, 1979]. This review speaks for the present importance, if not dominance, of indeterminism, and against this background there is the unmistakable possibility of a reversion in the future to forms of imprecise, linguistic models of behavior [Camara et al., 1985]. This is being done under the objective of improving communication and because now (this time around) the manipulation and exploitation of such models can be expected to be profoundly changed by recent developments in the methods and programming languages of artificial intelligence [Duda and Shortliffe, 1983].

If this is so, it will change many of the conventions either assumed or set up in this review. The original motivation for the review, it has to be said, was a concern with the correctness of the convention that the more detailed and "comprehensive" the model, the more "scientific" would be the explanation of past behavior and the more accurate the prediction of future behavior. In the end, the conventional assumptions made here about the application of the scientific method may themselves have to be overturned, either as a consequence of philosophical inquiry [Chalmers, 1982] or as a result of the challenge of modern computer technology and artificial intelligence. What is it, we will have to ask ourselves, that lies behind the impressive color graphics that tell us that water quality will be "disgusting" as a result of some contemplated action?

\section{NOTATION}

$a$ (subscript) augmented (state-parameter) system properties.

$B$ matrices of sensitivity coefficients $\left[\partial x_{i} / \partial x_{j}\left(t_{f}\right)\right]$, $\left[\partial x_{i} / \partial \alpha_{j}\right],\left[\partial x_{i} / d u_{j}\right],\left[\partial x_{i} / \partial \xi_{j}\right]$.

$C$ matrix of sensitivity coefficients $\left[\partial y_{i} / \partial \alpha_{j}\right]$.

e error of mismatch between model response and observed response.

$F \quad$ system matrix for a linear system.
$G$ input matrix for a linear system.

$H$ observations matrix for a linear system.

$J$ squared-error criterion for parameter estimation.

$K$ Kalman gain matrix.

$P_{a} \quad$ variance-covariance matrix of (state-parameter) estimation errors.

$P^{c} \quad$ covariance matrix of state and parameter estimation errors.

$P^{P} \quad$ variance-covariance matrix of parameter estimation errors.

$P^{s} \quad$ variance-covariance matrix of state estimation errors.

$Q_{a}$ variance-covariance matrix of augmented (stateparameter) system disturbances.

$Q^{P} \quad$ variance-covariance matrix of parameter disturbances.

$Q^{s} \quad$ variance-covariance matrix of state disturbances.

$r$ three-dimensional, orthogonal spatial directions.

$R$ variance-covariance matrix of output measurement errors.

$S$ variance-covariance matrix of input measurement errors.

$t$ time.

$t_{f}$ beginning of prediction period.

$t_{k} \quad k$ th discrete instant in time.

$t_{N}$ end of identification period.

$t_{p}$ end of prediction period.

$t_{0}$ beginning of identification period.

u measured input disturbances of the system.

$W$ weighting matrix.

$\mathbf{x}$ state variables.

$\mathbf{x}_{a}$ augmented state-parameter vector.

$\mathbf{x}^{*}$ instrumental variable vector.

y measured output response variables.

$\alpha$ vector of model parameters (class II model).

B vector of model parameters (class III model).

$\xi$ unmeasured input disturbances of the system.

$v$ innovations process errors, i.e., one-step-ahead prediction errors.

$\omega$ lumped errors of a class III model representation.

$\boldsymbol{\theta}$ vector of model parameters (class I model).

$\zeta$ parametric disturbances.

$\Psi$ gain matrix in batch estimation algorithm.

$\Phi$ state transition matrix.

Accents are used to denote the following: overbar, nominal, or reference, values of the states, parameters, inputs, or outputs; tilde, errors (or small perturbations) associated with the states, parameters, inputs, or outputs. Angle brackets denote estimated (or predicted) values of the states, parameters, inputs, or outputs. (In some figures, estimates are denoted by a circumflex.)

Acknowledgments. Much of the work leading to the preparation of this review was conducted while the author was with the International Institute for Applied Systems Analysis (IIASA) at Laxenburg in Austria. It is hard to quantify the influence of the uniquely stimulating intellectual environment that IIASA has provided; it is much easier to acknowledge one's deep debt to it. I am also indebted to Ken Reckhow and to the five anonymous persons whose "thankless" task it was to review the original version of the paper. It seems small reward to thank them here for the personal benefits I gained from their exhaustive criticisms. One further person, Peter Young, must be singled out for my special acknowledgment. Finally, thanks are due to Frances Donnelly for the preparation of the typescript.

\section{REFERENCES}

Alcamo, J., and J. Bartnicki, An approach to uncertainty of a long range air pollutant transport model, Working Pap. WP-85-88, Int. Inst. for Appl. Syst. Anal., Laxenburg, Austria, 1985. 
Al-Dahan, M. I., M. S. Leaning, E. R. Carson, D. W. Hill, and L. Finkelstein, The validation of complex, unidentifiable models of the cardiovascular system, Identification and System Parameter Estimation, edited by H. A. Barker and P. C. Young, pp. 1213-1218, Pergamon, New York, 1985.

Argentesi, F., and L. Olivi, Statistical sensitivity analysis of a simulation model for the biomass-nutrient dynamics in aquatic ecosystems, Proceedings, 4th Summer Computer Simulation Conference, pp. 389-393, Simulation Counc., La Jolla, Calif., 1976.

Åström, K. J., Maximum likelihood and prediction error methods, Automatica, 16, 551-574, 1980

Åström, K. J., and T. Bohlin, Numerical identification of linear dynamic systems from normal operating records, in Theory of SelfAdaptive Control Systems, edited by P. H. Hammond, pp. 96-111, Plenum, New York, 1966.

Åström, K. J., and P. Eykhoff, System identification-A survey, Automatica, 7, 123-162, 1971.

Bagchi, A., R. Strijbos, and G. Thé, Identification of a distributed parameter system with boundary noise, Int. J. Syst. Sci., 11, 49-56, 1980.

Barker, H. A., and P. C. Young (Eds.), Identification and System Parameter Estimation, Pergamon, New York, 1985.

Beck, M. B., The identification of algal population dynamics in a freshwater stream, in Computer Simulation of Water Resources Systems, edited by G. C. Vansteenkiste, pp. 483-494, North-Holland, Amsterdam, 1975.

Beck, M. B., Random signal analysis in an environmental sciences problem, Appl. Math. Modell., 2, 23-29, 1978.

Beck, M. B., Model structure identification from experimental data, in Theoretical Systems Ecology: Advances and Case Studies, edited by E. Halfon, pp. 259-289, Academic, Orlando, Fla., $1979 a$.

Beck, M. B., System identification, estimation, and forecasting of water quality, I, Theory, Working Pap. WP-79-31, Int. Inst. for Appl. Syst. Anal., Laxenburg, Austria, 1979b.

Beck, M. B., Operational estimation and prediction of nitrification dynamics in an activated sludge process, Water Res., 15, 13131330, 1981a.

Beck, M. B., Hard or soft environmental systems?, Ecol. Modell., 11, 237-251, $1981 b$.

Beck, M. B., Identifying models of environmental systems' behaviour, Math. Modell., 3, 467-480, 1982.

Beck, M. B., Uncertainty, system identification and the prediction of water quality, in Uncertainty and Forecasting of Water Quality, edited by M. B. Beck and G. van Straten, pp. 3-68, Springer Verlag, New York, 1983.

Beck, M. B., Water Quality Management: A Review of the Development and Application of Mathematical Models, Lect. Notes in Eng., vol. 11, Springer Verlag, New York, 1985 a.

Beck, M. B., Lake eutrophication: Identification of tributary nutrient loading and sediment resuspension dynamics, Appl. Math. Comput., $17,433-458,1985 b$.

Beck, M. B., The analysis of environmental time-series, Statistica e Risorse Naturali, annual conference, pp. 67-90, Soc. Italiana di Statist., Giardini Naxos, Sicily, $1985 c$.

Beck, M. B., Structures, failure, inference, and prediction, in Identification and System Parameter Estimation, edited by H. A. Barker and P. C. Young, pp. 1443-1448, Pergamon, New York, 1985d.

Beck, M. B., Identification, estimation, and control of biological wastewater treatment processes, IEE Proc., Ser. D, 133(5), 254-264, 1986.

Beck, M. B., and P. C. Young, Systematic identification of DO-BOD model structure, Proc. Am. Soc. Civ. Eng. J. Environ. Eng. Div., 102(EE5), 902-927, 1976

Beck, M. B., E. Halfon, and G. van Straten, The propagation of errors and uncertainty in forecasting water quality, I, Method, Working Pap. WP-79-100, Int. Inst. for Appl. Syst. Anal., Laxenburg, Austria, 1979.

Beer, T., and P. C. Young, Longitudinal dispersion in natural streams, J. Environ. Eng., 109(5), 1049-1067, 1983.

Bellman, R. E., and K. J. Åström, On structural identifiability, Math. Biosci., 7, 329-339, 1970.

Bellman, R. E., and R. E. Kalaba, Quasilinearisation and Nonlinear Boundary Value Problems, Elsevier, New York, 1965.

Bellman, R. E., H. Kagiwada, and R. Kalaba, Inverse problems in ecology, J. Theor. Biol., 11, 164-167, 1966.

Benjamin, J. R., and C. A. Cornell, Probability, Statistics, and Decision for Civil Engineers, McGraw-Hill, New York, 1970.
Benson, M. Parameter fitting in dynamic models, Ecol. Modell., 6, 97-115, 1979.

Bierman, V. J., D. M. Dolan, E. F. Stoermer, J. E. Gannon, and V. E. Smith, The development and calibration of a spatially simplified multiclass phytoplankton model for Saginaw Bay, Lake Huron, Contrib. 33, Great Lakes Environ. Plann. Study, Large Lakes Res. Sta., U.S. Environ. Prot. Agency, Grosse, Ile, Mich., 1980.

Bossermann, R. W., and R. K. Ragade, Ecosystem analysis using fuzzy set theory, Ecol. Modell., 16, 191-208, 1982.

Botkin, D. B., Bits, bytes, and IBP, Bioscience, 27, 385-389, 1977.

Bowles, D. S., and W. J. Grenney, Steady-state river quality modelling by sequential extended Kalman filters, Water Resour. Res., 14 $84-95,1978 a$.

Bowles, D. S., and W. J. Grenney, Estimation of diffusive loading of water quality pollutants by Kalman filtering, in Applications of Kalman Filter to Hydrology, Hydraulics, and Water Resources, edited by C.-L. Chiu, pp. 581-597, Stochastic Hydraul. Program, Univ. of Pittsburgh, $\mathrm{Pa} ., 1978 b$.

Box, G. E. P., and G. M. Jenkins, Time Series Analysis, Forecasting and Control, Holden-Day, San Francisco, Calif., 1970.

Brush, S. G., The chimerical cat: Philosophy of quantum mechanics in perspective, Soc. Stud. Sci., 10(4), 393-447, 1980.

Budgell, W. P., A dynamic-stochastic approach for modelling advection-dispersion processes in open channels, in Time Series Methods in Hydrosciences, edited by A. H. El-Sharaawi and S. R. Esterby, pp. 244-263, Elsevier, New York, 1982.

Burges, S. J., and D. P. Lettenmaier, Probabilistic methods in stream quality management, Water Resour. Bull., 11, 115-130, 1975.

Burns, J. R., Error analysis of nonlinear simulations: Applications to world dynamics, IEEE Trans. Syst. Man Cybern., 5(3), 331-340, 1975.

Cale, W. G., Jr., and P. L. Odell, Concerning aggregation in ecosystem modeling, in Theoretical Systems Ecology: Advances and Case Studies, edited by E. Halfon, pp. 283-298, Academic, Orlando, Fla., 1979

Cale, W. G., Jr., R. V. O'Neill, and H. H. Shugart, Development and application of desirable ecological models, Ecol. Modell., 18, 171 186, $1983 a$.

Cale, W. G., Jr., R. V. O'Neill, and R. H. Gardner, Aggregation error in nonlinear ecological models, J. Theor. Biol., 100, 539-550, 1983 b.

Camara, A. S., M. D. Pinheiro, M. P. Antunes, and M. J. Seixas, Linguistic simulation in planning theory and application, paper presented at the International Conference of the System Dynamics Society, Keystone, Colo., July 1985.

Canada Centre for Inland Waters, Assessment of water quality simulation capability for Lake Ontario, Sci. Ser. 111, Can. Cent. for Inland Waters, Burlington, Ont., 1979.

Canale, R. P., L. M. De Palma, and W. F. Powers, Sampling strategies for water quality in the Great Lakes, Rep. EPA-600/3-80-55, U.S. Environ. Prot. Agency, Environ. Res. Lab., Duluth, Minn. 1980.

Caswell, H., The validation problem, in Systems Analysis and Simulation in Ecology, edited by B. C. Patten, vol. IV, pp. 313-325, Academic, Orlando, Fla., 1976.

Chadderton, R. A., A. C. Miller, and A. J. McDonnell, Uncertainty analysis of dissolved oxygen model, Proc. Am. Soc. Civ. Eng. J. Environ. Eng. Div., 108(EE 5), 1003-1013, 1982.

Chalmers, A. F., What Is This Thing Called Science?, 2nd ed., The Open University Press, Milton Keynes, England, 1982.

Chan, S. K. C., Wind-induced resuspension of sediment in a shallow lake, M.Sc. thesis, Dep. of Civ. Eng., Imperial Coll., London, 1984.

Chapra, S. C., and K. H. Reckhow, Engineering Approaches for Lake Management, vol. 2, Mechanistic Modeling, Butterworths, Boston, Mass., 1983.

Chen, C. W., and D. J. Smith, Preliminary insights into a threedimensional ecological-hydrodynamical model, in Perspectives in Lake Ecosystem Modelling, edited by D. Scavia and A. Robertson, pp. 249-279, Ann Arbor Science, Ann Arbor, Mich., 1979.

Chuang, P. H., and J. Munro, Linear programming with imprecise data, Civ. Eng. Syst., 1, 37-41, 1983.

Cobelli, C., A. Lepschy, and G. Romanin-Jacur, Structural identifiability of linear compartmental models of ecosystems, in Theoretical Systems Ecology, edited by E. Halfon, pp. 237-258, Academic, Orlando, Fla., 1979.

Collins, C. D., Formulation and validation of a mathematical model of phytoplankton growth, Ecology, 61(3), 639-649, 1980.

Constable, T. W., and E. A. McBean, Kalman filter modelling of 
Speed River quality, Proc. Am. Soc. Civ. Eng. J. Environ. Eng. Div., 105, 961-978, 1979.

Cosby, B. J., Dissolved oxygen dynamics of a stream: Model discrimination and estimation of parameter variability using an extended Kalman filter, Water Sci. Technol, 16(5-7), 561-569, 1984.

Cosby, B. J., and G. M. Hornberger, Identification of photosynthesislight models for aquatic systems, I, Theory and simulations, Ecol. Modell., 23, 1-24, 1984.

Cosby, B. J., G. M. Hornberger, and M. G. Kelly, Identification of photosynthesis-light models for aquatic systems, II, Application to a macrophyte dominated stream, Ecol. Modell., 23, 25-51, 1984.

DeLucia, R., and E. McBain, Model of the Saint John River, United States, in Models for Water Quality Management, edited by A. K. Biswas, pp. 91-127, McGraw-Hill, New York, 1981.

Detchmendy, D. M., and R. Sridhar, On the experimental determination of the dynamical characteristics of physical systems, Proceedings, U.S. National Electronics Conference, pp. 575-580, 1965.

Dettinger, M. D., and J. L. Wilson, First order analysis of uncertainty in numerical models of groundwater flow, I, Mathematical development, Water Resour. Res., 17(1), 149-161, 1981.

Di Cola, G., L. Guerri, and H. Verheyden, Parametric estimation in a compartmental aquatic ecosystem, in Identification and System $\mathrm{Pa}$ rameter Estimation, part 2, pp. 157-165, Institute of Control Sciences, Moscow, 1976.

Di Toro, D. M., and G. van Straten, Uncertainty in the parameters and predictions of phytoplankton models, Working Pap. WP-79-27, Int. Inst. for Appl. Syst. Anal., Laxenburg, Austria, 1979.

Dorf, R. C., Time-Domain Analysis and Design of Control Systems, Addison-Wesley, Reading, Mass., 1965.

Duda, R. O., and E. H. Shortliffe, Expert systems research, Science, 220, 261-268, 1983.

Durbin, J., Time series analysis, J. R. Stat. Soc., Ser. A, 147(2), 161173, 1984.

Ellis, J. C., and R. F. Lacey, Sampling: Defining the task and implementing the scheme, in River Pollution Control, edited by M. J. Stiff, pp. 247-274, Ellis Horwood, Chichester, England, 1980.

Eykhoff, P., System Identification-Parameter and State Estimation, John Wiley, New York, 1974.

Fedra, K., Pelagic foodweb analysis: Hypothesis testing by simulation, Kiel. Meeresforsch., 5, 240-258, 1981.

Fedra, K., Environmental modeling under uncertainty: Monte Carlo simulation, Res. Rep. RR-83-28, Int. Inst. for Appl. Syst. Anal., Laxenburg, Austria, 1983.

Fedra, K., Advanced decision-oriented software for the management of hazardous substances, I, Structure and design, Collab. Pap. CP85-18, Int. Inst. for Appl. Syst. Anal., Laxenburg, Austria, 1985.

Fedra, K., and D. P. Loucks, Interactive computer technology for planning and policy modeling, Water Resour. Res., 21(2), 114-122, 1985.

Fedra, K., G. van Straten, and M. B. Beck, Uncertainty and arbitrariness in ecosystems modelling: A lake modelling example, Ecol. Modell., 13, 87-110, 1981.

Feyerabend, P. K., Against Method: Outline of an Anarchistic Theory of Knowledge, New Left Books, London, 1975.

Finney, B. A., D. S. Bowles, and M. P. Windham, Random differential equations in river water quality modelling, Water Resour. Res., 18(1), 122-134, 1982.

Fisher, I. H., Uncertainty and dynamic policies for the control of nutrient inputs to lakes, in Uncertainty and Forecasting of Water Quality, edited by M. B. Beck and G. van Straten, pp. 357-370, Springer Verlag, New York, 1983.

Flood, R. L., M. S. Leaning, D. G. Cramp, and E. R. Carson, Clinical time-series: Analysis, modelling and recursive estimation, in Identification and System Parameter Estimation, edited by H. A. Barker and P. C. Young, pp. 1613-1618, Pergamon, New York, 1985.

Fontaine, T. D., Application of risk and uncertainty analysis techniques to a heavy metal speciation model, Ecol. Modell., 22, 101108, 1984.

Fontaine, T. D., and B. M. Lesht, Contaminant management strategies for the Great Lakes: Optimal solutions under uncertain conditions, report, NOAA Great Lakes Environ. Res. Lab., Ann Arbor, Mich., 1986.

Forsyth, R. (Ed.), Expert Systems: Principles and Case Studies, Chapman and Hall, London, 1984.

Gardner, R. H., and R. V. O'Neill, Parameter uncertainty and model predictions: A review of Monte Carlo results, in Uncertainty and
Forecasting of Water Quality, edited by M. B. Beck and G. van Straten, pp. 245-257, Springer Verlag, New York, 1983.

Gardner, R. H., R. V. O'Neill, J. B. Mankin, and D. Kumar, Comparative error analysis of six predator-prey models, Ecology, 61(2), 323-332, 1980a.

Gardner, R. H., D. D. Huff, R. V. O'Neill, J. B. Mankin, J. H. Carney, and J. Jones, Application of error analysis to a marsh hydrology model, Water Resour. Res., 16, 659-664, $1980 b$.

Gardner, R. H., R. V. O'Neill, J. H. Carney, and J. B. Mankin, A comparison of sensitivity analysis and error analysis based on a stream ecosystem model, Ecol. Modell., 12, 173-190, 1981a.

Gardner, R. H., R. V. O'Neill, and J. H. Carney, Spatial patterning and error propagation in a stream ecosystem model, Proceedings, Summer Computer Simulation Conference, pp. 391-395, Simulation Counc., La Jolla, Calif., $1981 b$.

Gardner, R. H., W. G. Cale, Jr., and R. V. O'Neill, Robust analysis of aggregation error, Ecology, 63(6), 1771-1779, 1982.

Gelb, A. (Ed.), Applied Optimal Estimation, MIT Press, Cambridge, Mass., 1974.

Gentil, S., A discrete model for the study of a lake, Appl. Math. Modell., 3, 193-198, 1979.

Gentil, S., Identifiability study of an aquatic ecosystem model, Int. J. Syst. Sci., 13(8), 881-895, 1982.

Gentil, S., Predictive or explanatory models for aquatic ecosystems, Water Sci. Technol., 16(5-7), 571-578, 1984.

Gentil, S., and D. Perrier, Practical identifiability study of an environmental model by a statistical analysis method, in Identification and System Parameter Estimation, edited by H. A. Barker and P. C. Young, pp. 235-240, Pergamon, New York, 1985.

Godfrey, K. R., and J. J. Distefano, Identifiability of model parameters, in Identification and System Parameter Estimation, edited by H. A. Barker and P. C. Young, pp. 89-114, Pergamon, New York, 1985.

Godfrey, K. R., R. P. Jones, R. F. Brown, and J. P. Norton, Factors affecting the identifiability of compartmental models, Automatica, 18(3), 285-293, 1982.

Goodall, D. W., Building and testing ecosystem models, in Mathematical Models in Ecology, edited by J. N. R. Jeffers, pp. 173-194, Blackwell Scientific, Oxford, 1972.

Goodwin, G. C., and R. L. Payne, Dynamic System IdentificationExperiment Design and Data Analysis, Academic, Orlando, Fla., 1977.

Gupta, V. K., and S. Sorooshian, Uniqueness and observability of conceptual rainfall-runoff model parameters: The percolation process examined, Water Resour. Res., 19(1), 269-276, 1983.

Gustavsson, I., Survey of applications of identification in chemical and physical processes, Automatica, 11, 3-24, 1975.

Halfon, E., Identification and ecological modelling, in Identification and System Parameter Estimation, part 2, pp. 91-97, Institute of Control Sciences, Moscow, 1976.

Halfon, E., Analytical solution of the system sensitivity equation associated with a linear model, Ecol. Modell., 3, 301-307, 1977.

Halfon, E., On the parameter structure of a large-scale ecological model, in Contemporary Quantitative Ecology and Related Ecometrics, edited by G. P. Patil and M. L. Rosenzweig, pp. 279-293, International Co-operative Publishing House, Fairland, Md., 1979.

Halfon, E., Error analysis and simulation of Mirex behaviour in Lake Ontario, Ecol. Modell., 22, 213-252, 1984.

Halfon, E., and R. J. Maguire, Distribution and transformation of fenitrothion sprayed on a pond: Modeling under uncertainty, in Uncertainty and Forecasting of Water Quality, edited by M. B. Beck and G. van Straten, pp. 117-128, Springer Verlag, New York, 1983.

Halfon, E., H. Unbehauen, and C. Schmid, Model order estimation and system identification theory and application to the modelling of ${ }^{32} \mathrm{P}$ kinetics within the trophogenic zone of a small lake, Ecol. Modell., 6, 1-22, 1979.

Harris, C. J., Modeling, simulation and control of stochastic systems with applications in wastewater treatment, Int. J. Syst. Sci., 8(4), 393-411, 1977.

Harvey, A. C., A unified view of statistical forecasting procedures, $J$. Forecasting, 3, 245-275, 1984.

Hendry, D. F., Time-series econometrics, J. R. Stat. Soc., Ser. A, $137(2), 327-339,1984$

Herbay, J.-P., Y. Smeers, and D. Tyteca, Water quality management with time-varying river flow and discharger control, Water Resour. Res., 19(6), 1478-1487, 1983. 
Hipel, K. W., Geophysical model discrimination using the Akaike information criterion, IEEE Trans. Automat. Contr., AC-26, 358$378,1981$.

Holling, C. S. (Ed.), Adaptive Environmental Assessment and Management, John Wiley, New York, 1978.

Holmberg, A., A systems engineering approach to biotechnical processes-Experiences of modelling, estimation and control methods, Acta Polytech. Scand. Math. Comput. Sci. Ser., no. 33, 1981.

Holmberg, A., and J. Ranta, Procedures for parameter and state estimation of microbial growth process models, Automatica, 18(2), 181193, 1982.

Hornberger, G. M., Uncertainty in dissolved oxygen prediction due to variability in algal photosynthesis, Water Res., 14, 355-361, 1980.

Hornberger, G. M., and B. J. Cosby, Evaluation of a model of longterm response of catchments to atmospheric deposition of sulphate, in Identification and System Parameter Estimation, edited by $\mathrm{H}$. A Barker and P. C. Young, pp. 229-234, Pergamon, New York, $1985 a$

Hornberger, G. M., and B. J. Cosby, Selection of parameter values in environmental models using sparse data: A case study, Appl. Math. Comput., 17(4), 335-355, 1985 b.

Hornberger, G. M., and R. C. Spear, Eutrophication in Peel Inlet, I, Problem-defining behaviour and a mathematical model for the phosphorous scenario, Water Res., 14, 29-42, 1980.

Hornberger, G. M., and R. C. Spear, An approach to the preliminary analysis of environmental systems, J. Environ. Manage., 12, 7-18, 1981.

Hornberger, G. M., K. J. Beven, B. J. Cosby, and D. E. Sappington, Shenandoah Watershed study: Calibration of a topography-based variable contributing area hydrological model to a small forested catchment, Water Resour. Res., 21(12), 1841-1850, 1985.

Isermann, R., Practical aspects of process identification, Automatica, $16,575-587,1980$

Isermann, R. (Ed.), System Identification, Int. Fed. for Automat. Contr. Tutorials, Pergamon, New York, 1981.

Jaffe, P. R., and R. A. Ferrara, Modeling sediment and water column interactions for hydrophobic pollutants: Parameter discrimination and model response to input uncertainty, Water Res., 18(9), 1169 $1174,1984$.

Jaffe, P. R., and F. L. Parker, Uncertainty analysis of first order decay model, J. Environ. Eng., 110(1), 131-140, 1984.

Jakeman, A. J., and P. C. Young, Toward optimal modelling of translocation data from tracer studies, Proceedings, 4th Biennial Conference, pp. 248-253, Simulation Soc. of Australia, 1980.

Jazwinski, A. H., Stochastic Processes and Filtering Theory, Academic, Orlando, Fla., 1970

Johnston, P. R., and D. Pilgrim, Parameter optimization for watershed models, Water Resour. Res., 12(3), 477-486, 1976.

Jolankai, G., and A. Szöllösi-Nagy, A simple eutrophication model for the Bay of Keszethely, Lake Balaton, Modelling the Water Quality of the Hydrological Cycle, Proceedings of the Baden Symposium, AISH-IAHS Publ., 125, pp. 137-150, 1978.

Jørgensen, S. E., H. E. Mejer, and M. Friis, Examination of a lake model, Ecol. Modell., 4, 253-278, 1978.

Jørgensen, S. E., L. A. Jørgensen, L. Kamp-Nielsen, and H. F. Mejer, Parameter estimation in eutrophication modelling, Ecol. Modell. 13, 111-129, 1981

Jowitt, P. W., Risk analysis, fuzzy logic, and river basin management, Water Sci. Technol., 16(5-7), 579-585, 1984.

Jowitt, P. W., and J. P. Lumbers, Water quality objectives, discharge standards, and fuzzy logic, Optimal Allocation of Water Resources, I AHS-AISH Publ., 135, 241-250, 1982.

Kohberger, R. C., D. Scavia, and J. W. Wilkinson, A method for parameter sensitivity analysis in differential equation models, Water Resour. Res., 14(1), 25-29, 1978.

Koivo, H. N., and A. J. Koivo, Least-squares estimator for polluted stream variables in a distributed parameter model, Adv. Water Resour., 1, 191-194, 1978

Koivo, A. J., and G. R. Phillips, Identification of mathematical models for DO and BOD concentrations in polluted streams from noise corrupted measurements, Water Resour. Res., 7(4), 853-862, 1971

Koivo, A. J., and G. R. Phillips, Optimal estimation of DO, BOD, and stream parameters using a dynamic discrete time model, Water Resour. Res., 12(4), 705-711, 1976.

Kremer, J. N., Ecological implications of parameter uncertainty in stochastic simulation, Ecol. Modell., 18, 187-207, 1983.
Kuhn, T. S., The Structure of Scientific Revolutions, University of Chicago Press, Ill., 1970.

Lack, T. J., and J. W. G. Lund, Observations and experiments on the phytoplankton of Blelham Tarn, English Lake District, Freshwater Biol., 4, 399-415, 1974.

Lang, I., Impact on policy-making: Background to a government decision, in Modeling and Managing Shallow Lake Eutrophication, edited by L. Somlyody and G. van Straten, pp. 110-121, Springer Verlag, New York, 1986.

Lee, E. S., and I. Hwang, Stream quality modelling by quasilinearisation, J. Water Pollut. Contr. Fed., 43(2), 306-317, 1971

Lettenmaier, D. P., and S. J. Burges, Use of state estimation techniques in water resource modelling, Water Resour. Bull., 12(1), 83-99, 1976.

Lettenmaier, D. P., and S. J. Burges, Design of trend monitoring networks, Proc. Am. Soc. Civ. Eng. J. Environ. Eng. Div., 103(EE5) 785-802, 1977.

Lettenmaier, D. P., and J. E. Richey, Use of first-order analysis in estimating mass balance errors and planning sampling activities, in Theoretical Systems Ecology: Advances and Case Studies, edited by E. Halfon, pp. 80-106, Academic, Orlando, Fla., 1979.

Lewandowski, A., Issues in model validation, Angew. Systemanal., 3(1), 2-11, 1982

Lewis, S., and A. Nir, A study of parameter estimation procedures of a model for lake phosphorus dynamics, Ecol. Modell., 4, 99-117, 1978.

Ljung, L., Asymptotic behaviour of the extended Kalman filter as a parameter estimator, IEEE Trans. Automat. Contr., 24, 36-50, 1979.

Ljung, L., and T. Söderström, Theory and Practice of Recursive Identification, MIT Press, Cambridge, Mass., 1983.

Loehle, C., Evaluation of theories and calculation tools in ecology, Ecol. Modell., 19, 239-247, 1983

Lohani, B. N., and A. R. Saleemi, Recent developments on stochastic programming model for water quality management, Water Supply Manage., 6(6), 511-520, 1982.

Lohani, B. N., and N. C. Thanh, Probabilistic water quality control policies, Proc. Am. Soc. Civ. Eng. J. Environ. Eng. Div., 105(EE4), 713-725, 1979.

Loucks, D. P., and W. R. Lynn, Probabilistic models for predicting stream quality, Water Resour. Res., 2(3), 593-605, 1966.

Loucks, D. P., J. Kindler, and K. Fedra, Interactive water resources modelling and model use: An overview, Water Resour. Res., 21(2), 95-102, 1985.

Maciejowski, J. M., Maximum likelihood estimation of initial conditions for unknown dynamical systems, in Identification and System Parameter Estimation, edited by R. Isermann, Pergamon, New York, 1980.

Malone, R. F., H. Saidi, and K. Wegener, Predictive accuracy determination applied to a linear model of phosphorus loading resulting from urban runoff, Appl. Math. Modell., 8, 81-88, 1984.

Mankin, J. B., R. V. O'Neill, H. H. Shugart, and B. W. Rust, The importance of validation in ecosystem analysis, in New Directions in the Analysis of Ecological Systems, Proc. Ser., vol. 5(1), edited by G. S. Innis, 63-72, Simulation Council, La Jolla, Calif., 1977

May, R. M., Simple mathematical models with very complicated dynamics, Nature, 261, 459-467, 1976.

McCrae, G. J., Methods for the sensitivity and uncertainty analysis of long-range transport models, Sources and Evaluation of Uncertainty in Long Range Transport Models, proceedings of a meeting, Am. Meteorol. Soc., Boston, Mass., in press, 1987.

McLaughlin, D. B., Parameter estimation problems in water resources modelling, in Modelling, Identification and Control in Environmental Systems, edited by G. C. Vansteenkiste, pp. 137-151, North-Holland, Amsterdam, $1978 a$.

McLaughlin, D. B., Potential applications of Kalman filtering concepts to groundwater basin management, in Applications of Kalman Filter to Hydrology, Hydraulics, and Water Resources, edited by C.-L. Chiu, pp. 639-655, Stochastic Hydraulics Program, University of Pittsburgh, $\mathrm{Pa} ., 1978 \mathrm{~h}$.

McLaughlin, D. B., Hanford groundwater modeling-A numerical comparison of Bayesian and Fisher parameter estimation techniques, Rep. RMO-C-24, Resour. Manage. Assoc., Lafayette, Calif., 1979

McLaughlin, D. B., Statistical analysis of uncertainty propagation and model accuracy, in Uncertainty and Forecasting of Water Quality, edited by M. B. Beck and G. van Straten, pp. 305-319, Springer Verlag, New York, 1983. 
McLaughlin, D. B., A distributed parameter state space approach for evaluating the accuracy of groundwater predictions, Ph.D. dissertation, Princeton Univ., N. J., 1985.

Mejer, H. F., and L. A. Jørgensen, Identification methods applied to two Danish lakes, in Uncertainty and Forecasting of Water Quality, edited by M. B. Beck and G. van Straten, pp. 173-182, Springer Verlag, New York, 1983.

Miller, D. R., Sensitivity analysis and validation of simulation models, J. Theor. Biol., 48, 345-360, 1974.

Miller, D. R., G. Butler, and C. Bramall, Validation of ecological system models, J. Environ. Manage., 4, 383-401, 1976.

Ministry of Public Housing, Physical Planning, and Environmental Protection, Handling uncertainty in environmental impact assessment, Milieu-Effect Rapportage, vol. 18, The Netherlands, 1985.

Moore, R. J, and D. A. Jones, Coupled Bayesian-Kalman filter estimation of parameters and states of dynamic water quality model, in Applications of Kalman Filter to Hydrology, Hydraulics, and Water Resources, edited by C.-L. Chiu, pp. 599-635, Stochastic Hydraulics Program, University of Pittsburgh, Pa., 1978.

Moore, S. F., Estimation theory applications to design of water quality monitoring systems, Proc. Am. Soc. Civ. Eng. J. Hydraul. Div., 99(HY5), 815-831, 1973.

Moore, S. F., Applications of Kalman filter to water quality studies, in Applications of Kalman Filter to Hydrology, Hydraulics and Water Resources, edited by C.-L. Chiu, pp. 485-507, Stochastic Hydraulics Program, University of Pittsburgh, Pa., 1978.

Moore, S. F., G. C. Dandy, and R. J. deLucia, Describing variance with a simple water quality model and hypothetical sampling programs, Water Resour. Res., 12(4), 795-804, 1976.

Munro, J., and D. L. Smith, Linear programming duality in plastic analysis and synthesis, in Computer Aided Structural Design, Proceedings of a Symposium, vol. I, pp. A1.22-A1.54, Peter Peregrinus, Stevenage, England, 1972.

Najarian, T. O., and J. L. Taft, Nitrogen-cycle model for aquatic systems: Analysis, Proc. Am. Soc. Civ. Eng. J. Environ. Eng. Div. 107(EE6), 1141-1156, 1981.

Najarian, T. O., P. J. Kaneta, J. L. Taft, and M. L. Thatcher, Application of nitrogen-cycle model to Manasquan estuary, J. Environ. Eng., 110(1), 190-207, 1984.

Neuman, S. P., and J. Carrera, Maximum likelihood adjoint-state finite-element estimation of groundwater parameters under steadyand nonsteady-state conditions, Appl. Math. Comput., 17(4), 405432, 1985.

O'Neill, R. V., Error analysis of ecological models, Radionuclides in Ecosystems, Conf. 710501, pp. 898-908, Natl. Tech. Inf. Serv., Springfield, Va., 1973.

O'Neill, R. V., Natural variability as a source of error in model predictions, in Systems Analysis of Ecosystems, edited by G. S. Innis and R. V. O'Neill, pp. 23-32, International Co-operative Publishing House, Fairland, Md., 1979.

O'Neill, R. V., and R. H. Gardner, Sources of uncertainty in ecological models, in Methodology in Systems Modelling and Simulation, edited by B. P. Zeigler, M. S. Elzas, G. J. Klir, and T. I. Oren, pp. 447-463, North-Holland, Amsterdam, 1979.

O'Neill, R. V., and B. Rust, Aggregation errors in ecological models, Ecol. Modell., 7, 91-105, 1979.

O'Neill, R. V., J. W. Elwood, and J. G. Hildebrand, Theoretical implication of spatial heterogeneity in stream ecosystems, in Systems Analysis of Ecosystems, edited by G. S. Innis and R. V. O'Neill, pp. 79-101, International Co-operative Publishing House, Fairland, Md., 1979.

O'Neill, R. V., R. H. Gardner, and J. B. Mankin, Analysis of parameter error in a nonlinear model, Ecol. Modell., 8, 297-311, 1980.

O'Neill, R. V., R. H. Gardner, and J. H. Carney, Parameter constraints in a stream ecosystem model: Incorporation of a priori information in Monte Carlo error analysis, Ecol. Modell., 16, 51-65, $1982 a$.

O'Neill, R. V., R. H. Gardner, and D. E. Weller, Chaotic models as representations of ecological systems, Am. Natur., 120(2), 259-263, $1982 b$.

O'Neill, R. V., R. H. Gardner, L. W. Barnthouse, G. W. Suter, S. G. Hildebrand, and C. W. Gehrs, Ecosystem risk analysis: A new methodology, Environ. Toxicol. Chem., 1, 167-177, 1982c.

O'Neill, R. V., S. M. Bartell, and R. H. Gardner, Patterns of toxicological effects in ecosystems: A modelling study, Environ. Toxicol., Chem., 2, 451-461, 1983.

Paldor, N., A. Nir, and S. Lewis, Use of simulation as an aid in parameter identification of a lake ecosystem, in Modelling, Identifcation, and Control in Environmental Systems, edited by G. C. Vansteenkiste, pp. 183-193, North-Holland, Amsterdam, 1978.

Park, R. A., et al., A generalized model for simulating lake ecosystems, Simulation, 23, 33-50, 1974.

Parker, R. A., Estimation of aquatic ecosystem parameters, Verh. Int. Ver. Limnol., 18, 257-263, 1972.

Pickup, G., Testing the efficiencies of algorithms and strategies for automatic calibration of rainfall-runoff models, Hydrol. Sci. Bull., 22, 257-274, 1977.

Pinter, J., J. Szabo, and L. Somlyody, Multiextremal optimization for calibrating water resources models, Environ. Software, in press, 1987.

Popper, K. R., The Logic of Scientific Discovery, Hutchinson, London, 1968.

Reckhow, K. H., Sampling designs for lake phosphorus budgets, in Establishment of Water Quality Monitoring Programs, Am. Water Resour. Symp. Proc., edited by L. G. Everett and K. D. Schmidt, pp. 285-306, American Water Resources Association, Urbana, Ill., 1978.

Reckhow, K. H., The use of a simple model and uncertainty analysis in lake management, Water Resour. Bull., 15, 601-611, 1979a.

Reckhow, K. H., Uncertainty analysis applied to Vollenweider's phosphorus loading criterion, J. Water Pollut. Contr. Fed., 51(8), 2123$2128,1979 b$.

Reckhow, K. H., A method for the reduction of lake model prediction error, Water Res., 17(8), 911-916, 1983.

Reckhow, K. H., and S. C. Chapra, Confirmation of water quality models, Ecol. Modell., 20, 113-133, 1983a.

Reckhow, K. H., and S. C. Chapra, The need for simple approaches for the estimation of lake model prediction uncertainty, in Uncertainty and Forecasting of Water Quality, edited by M. B. Beck and G. van Straten, pp. 293-303, Springer Verlag, New York, $1983 b$.

Rinaldi, S., and R. Soncini-Sessa, Sensitivity analysis of generalised Streeter-Phelps models, Adv. Water Resour., 1, 141-146, 1978.

Rinaldi, S., R. Soncini-Sessa, H. Stehfest, and H. Tamura, Modelling and Control of River Quality, McGraw-Hill, New York, 1979a.

Rinaldi, S., P. Romano, and R. Soncini-Sessa, Parameter estimation of Streeter-Phelps models, Proc. Am. Soc. Civ. Eng. J. Environ. Eng. Div., 105(EE1), 75-88, $1979 b$.

Roberts, G. F., and F. DiCesare, A systems engineering methodology for structuring and calibrating lake ecosystem models, IEEE Trans. Syst. Man. Cybern., SMC12(1), 3-14, 1982.

Scavia, D., Uncertainty analysis of a lake eutrophication model, Ph.D. dissertation, Univ. of Mich., Ann Arbor, 1980.

Scavia, D., R. P. Canale, W. F. Powers, and J. F. Moody, Variance estimates for a dynamic eutrophication model of Saginaw Bay, Lake Huron, Water Resour. Res., 17(4), 1115-1124, 1981 a.

Scavia, D., W. F. Powers, R. P. Canale, and J. L. Moody, Comparison of first-order error analysis and Monte Carlo simulation in timedependent lake eutrophication models, Water Resour. Res., 17(4), 1051-1069, 1981b.

Schindler, D. W., and E. J. Fee, Experimental lakes area: Whole-lake experiments in eutrophication, J. Fish. Res. Board Can., 31, 937953,1974

Schindler, D. W., E. J. Fee, and T. Ruszczynski, Phosphorus input and its consequences for phytoplankton standing crop and production in the experimental lakes area and similar lakes, J. Fish. Res. Board Can., 35, 190-196, 1978.

Schrader, B. D., and S. F. Moore, Kalman filtering in water quality modeling: Theory vs, practice, Proceedings (1977) Winter Simulation Conference, edited by H. J. Highland, R. G. Sargent, and J. W. Schmidt, vol. 2, pp. 504-510, Simulation Counc., La Jolla, Calif., 1977.

Schweppe, F. C., Uncertain Dynamic Systems, Prentice-Hall, Englewood Cliffs, N. J., 1973.

Schweppe, F. C., Model identification problems, in Applications of Kalman Filter to Hydrology, Hydraulics and Water Resources, edited by C.-L. Chiu, pp. 115-134, Stochastic Hydraulics Program, University of Pittsburgh, Pa., 1978.

Sharefkin, M., Uncertainty and forecasting of water quality: Reflections of an ignorant Bayesian, in Uncertainty and Forecasting of Water Quality, edited by M. B. Beck and G. van Straten, pp. 373379, Springer Verlag, New York, 1983.

Shastry, J. S., L. T. Fan, and L. E. Erickson, Non-linear parameter estimation in water quality modelling, Proc. Am. Soc. Civ. Eng. J. Environ. Eng. Div., 99(EE3), 315-331, 1973. 
Söderström, T., On model structure testing in system identification, Int. J. Contr., 26, 1-18, 1977.

Söderström, T., and P. Stoica, Instrumental Variable Methods for System Identification, Springer Verlag, New York, 1983.

Söderström, T., L. Ljung, and I. Gustavsson, A theoretical analysis of recursive identification methods, Automatica, 14, 231-244, 1978.

Somlyody, L., Preliminary study on wind induced interaction between water and sediment for Lake Balaton, Proceedings of the Second Joint MTA/IIASA Task Force Meeting on Lake Balaton Modelling, vol. I, pp. 26-49, Int. Inst. for Appl. Syst. Anal., Laxenburg, Austria, 1980 .

Somlyody, L., Input data uncertainty and parameter sensitivity in a lake hydrodynamic model, in Uncertainty and Forecasting of Water Quality, edited by M. B. Beck and G. van Straten, pp. 129-155, Springer Verlag, New York, 1983.

Somlyody, L., Wind induced sediment resuspension in shallow lakes, in Water Quality Modelling in the Inland Natural Environment, pp. 287-298, British Hydraulics Research Association, Cranfield, England, 1986

Somlyody, L., and G. van Straten (Eds.), Modeling and Managing Shallow Lake Eutrophication, Springer Verlag, New York, 1986.

Somlyody, L., and R. J. B. Wets, Stochastic optimization models for lake eutrophication management, Collab. Pap. CP-85-16, Int. Inst. for Appl. Syst. Anal., Laxenburg, Austria, 1985.

Sorooshian, S., and V. K. Gupta, Automatic calibration of conceptua rainfall-runoff models: The question of parameter observability and uniqueness, Water Resour. Res., 19(1), 260-268, 1983.

Sorooshian, S., and V. K. Gupta, The analysis of structural identifiability: Theory and application to conceptual rainfall-runoff models, Water Resour. Res., 21(4), 487-496, 1985.

Sorooshian, S., V. K. Gupta, and J. L. Fulton, Evaluation of maximum likelihood parameter estimation techniques for conceptua rainfall-runoff models: Influence of calibration data variability and length on model credibility, Water Resour. Res., 19(1), 251-259, 1983.

Spear, R. C., The application of Kolmogorov-Renyi statistics to problems of parameter uncertainty in systems design, Int. J. Contr., 11, $771-778,1970$.

Spear, R. C., and G. M. Hornberger, Eutrophication in Peel Inlet, II, Identification of critical uncertainties via generalised sensitivity analysis, Water Res., 14, 43-49, 1980.

Spillers, W. P., Automated Structural Analysis: An Introduction, Pergamon, New York, 1972.

Stehfest, H., Mathematical modelling of self-purification of rivers, Prof. Pap. PP-77-11, Int. Inst. for Appl. Syst. Anal., Laxenburg, Austria, 1977.

Steinhorst, R. K., Parameter identifiability, validation, and sensitivity analysis of large system models, in Systems Analysis of Ecosystems, edited by G. S. Innis and R. V. O'Neill, pp. 33-58, International Co-operative Publishing House, Fairland, Md., 1979.

Streeter, H. W., and E. B. Phelps, A study of the pollution and natural purification of the Ohio River, Bull. 146, U.S. Public Health Serv. Washington, D. C., 1925.

Tamura, H., On some identification techniques for modelling river quality dynamics with distributed lags, in Handbook of Large-Scale Systems Engineering Applications, edited by M. G. Singh and A Titli, pp. 274-294, Amsterdam, 1979.

Thé, G., Parameter identification in a model for the conductivity of a river based on noisy measurements at two locations, in Modeling, Identification and Control in Environmental Systems, edited by G. C. Vansteenkiste, pp. 823-830, North-Holland, Amsterdam, 1978.

Thomann, R. V., Verification of water quality models, Proc. Am. Soc. Civ. Eng. J. Environ. Eng. Div., 108(EE5), 923-940, 1982.

Thomann, R. V., and R. P. Winfield, On the verification of a threedimensional phytoplankton model of Lake Ontario, Environmental Modeling and Simulation, Proceedings of a conference, Rep. EPA600/9-76-016, U.S. Environ. Prot. Agency, Washington, D. C., 1976.

Thomann, R. V., D. J. O'Connor, and D. M. Di Toro, Preliminary model of Potomac Estuary phytoplankton, Proc. Am. Soc. Civ. Eng. J. Environ. Eng. Div., 100(EE2), 699-715, 1974.

Thomann, R. V., R. P. Winfield, and J. J. Segna, Verification analysis of Lake Ontario and Rochester Embayment three-dimensional eutrophication models, Rep. EPA-600/3-79-094, U.S. Environ. Prot. Agency, Environ. Res. Lab., Duluth, Minn., 1979.

Tiwari, J. L., A modelling approach based on stochastic differential equations, the principle of maximum-entropy, and Bayesian infer- ence of parameters, in Compartmental Analysis of Ecosystem Models, edited by J. M. Matis, B. C. Patten, and G. C. White, pp. 167-194, International Co-operative Publishing House, Fairland, Md., 1979.

Tong, R. M., An analysis of fuzzy models and a discussion of their limitations, Rep. CUED/F-CAMS/TR 173, Univ. Eng. Dep., Cambridge, England, 1978.

Vajda, S., P. Valko, and A. Yermakova, A multistage parameter estimation procedure for continuous systems, in Identification and System Parameter Estimation, edited by H. A. Barker and P. C. Young, pp. 1579-1584, Pergamon, New York, 1985.

van Straten, G., Analysis of model and parameter uncertainty in simple phytoplankton models for Lake Balaton, in Progress in Ecological Engineering and Management by Mathematical Modelling, pp. 107-134, edited by D. M. Dubois, Editions CEBEDOC, Liege, Belgium, 1981.

van Straten, G., Maximum likelihood estimation of parameters and uncertainty in phytoplankton models, in Uncertainty and Forecasting of Water Quality, edited by M. B. Beck and G. van Straten, pp. 157-17i, Springer Verlag, New York, 1983.

van Straten, G., Analytical methods for parameter-space delimitation and application to shallow-lake phytoplankton-dynamics modeling, Appl. Math. Comput., 17, 459-482, 1985.

van Straten, G., and B. de Boer, Sensitivity to uncertainty in a phytoplankton-oxygen model for lowland streams, Working Pap. WP-79-28, Int. Inst. for Appl. Syst. Anal., Laxenburg, Austria, 1979.

van Straten, G., and S. Herodek, Estimation of algal growth parameters from vertical primary production profiles, Ecol. Modell., 15, $287-311,1982$

van Straten, G., R. H. B. Kersten, and G. P. J. Dijkema, Estimation of advection-dispersion characteristics of a stream from two-point time-series data, in Identification and System Parameter Estimation, edited by H. A. Barker and P. C. Young, pp. 249-254, Pergamon, New York, 1985

Vialas, C., A. Cheruy, and S. Gentil, An experimental approach to improve the Monod model identification, in Modelling and Control of Biotechnological Processes, edited by A. Johnson, pp. 155-159, Pergamon, New York, 1985.

Walker, W. W., A sensitivity analysis and error analysis framework for lake eutrophication modelling, Water Resour. Bull., 18, 53-60, 1982.

Ward, R. C., and J. C. Loftis, Incorporating the stochastic nature of water quality into management, J. Water Pollut. Contr. Fed., 55(4), 408-414, 1983.

Warn, A. E., and J. S. Brew, Mass balance, Water Res., 14, 1427-1434, 1980.

Warn, A. E., and P. J. Matthews, Calculation of the compliance of discharges with emission standards, Water Sci. Technol., 16(5-7), 183-196, 1984

Water Pollution Control Federation, No more grants, better water quality measures, no national non-point source programs now, WPCF Position Papers, J. Water Pollut. Contr. Fed., 53(4), 430$443,1981$.

Wheater, H. S., R. Abeliuk, and R. Perry, Parameter identification in models of unsaturated solute transport, in Water Quality Modelling in the Inland Natural Environment, pp. 171-185, British Hydraulics Research Association, Cranfield, 1986.

White, G. C., and G. M. Clark, Estimation of parameters for stochastic compartment models, in Compartmental Analysis of Ecosystem Models, edited by J. H. Matis, B. C. Patten, and G. C. White, pp. 134-144, International Co-operative Publishing House, Fairland, Md., 1979.

Whitehead, P. G., Applications of recursive estimation techniques to time variable hydrological systems, J. Hydrol., 40, 1-16, 1979.

Whitehead, P. G., An instrumental variable method of estimating differential-equation models of dispersion and water quality in nontidal rivers, Ecol. Modell., 9, 1-14, 1980.

Whitehead, P. G., Modeling and forecasting water quality in nontidal rivers: The Bedford Ouse Study, in Uncertainty and Forecasting of Water Quality, edited by M. B. Reck and G. van Straten, pp. 321-337, Springer Verlag, New York, 1983.

Whitehead, P. G., and G. M. Hornberger, Modelling algal behaviour in the River Thames, Water Res., 18(8), 945-953, 1984.

Whitehead, P. G., and P. C. Young, A dynamic stochastic model for water quality in part of the Bedford-Ouse River system, in Com- 
puter Simulation of Water Resources Systems, edited by G. C. Vansteenkiste, pp. 417-439, North-Holland, Amsterdam, 1975.

Whitehead, P. G., and P. C. Young, Water quality in river systems Monte Carlo analysis, Water Resour. Res., 15(6), 1305-1312, 1979.

Whitehead, P. G., M. B. Beck, and P. E. O'Connell, A systems model of streamflow and water quality in the Bedford Ouse River system, 2, Water quality modelling, Water Res., 15, 1157-1171, 1981.

Whitehead, P. G., C. Neal, S. Seden-Perriton, N. Christophersen, and S. Langan, A time-series approach to modelling stream acidity, $J$. Hydrol., 85, 281-303, 1986.

Yeh, W. W.-G., Review of parameter identification procedures in groundwater hydrology: The inverse problem, Water Resour. Res., 22(2), 95-108, 1986.

Yih, S.-M., and B. Davidson, Identification in nonlinear, distributed parameter water quality models, Water Resour. Res., 11(5), 693704, 1975.

Young, P. C., A recursive approach to time-series analysis, Bull. Inst. Math. Appl., 10, 209-224, 1974.

Young, P. C., General theory of modelling for badly defined systems, in Modeling, Identification and Control in Environmental Systems, edited by G. C. Vansteenkiste, pp. 103-135, North-Holland, Amsterdam, 1978.

Young, P. C., Self-adaptive Kalman filter, Electron. Lett., 15(2), 358 $360,1979$.

Young, P. C., Parameter estimation for continuous-time models-A survey, Automatica, 17(1), 23-39, 1981.

Young, P. C., The validity and credibility of models for badly defined systems, in Uncertainty and Foreçasting of Water Quality, edited by M. B. Beck and G. van Straten, pp. 69-98, Springer Verlag, New York, 1983.
Young, P. C., Recursive Estimation and Time-Series Analysis: An Introduction, Springer Verlag, New York, 1984.

Young, P. C., and A. J. Jakeman, Refined instrumental variable methods of recurşive time-series analysis, III, Extensions, Int. J. Contr., 3l(4), 741-764, 1980.

Young, P. C., and S. G. Wallis, The aggregated dead zone (ADZ) model for dispersion in rivers, in Water Quality Modelling in the Inland Natural Environment, pp. 421-433, British Hydraulics Research Association, Cranfield, England, 1986.

Young, P. C., and P. G. Whitehead, A recursive approach to timeseries analysis for multivariable systems, Int. J. Contr., 25, 457-482, 1977.

Young, P. C., G. M. Hornberger, and R. C. Spear, Modelling badly defined systems-Some further thoughts, Proceedings, SIMSIG Simulation Conference, pp. 24-32, Australian Natl. Univ., Canberra, 1978.

Young, P. C., A. J. Jakeman, and R. McMurtrie, An instrumental variable method for model order identification, Automatica, 16 , 281-294, 1980.

M. B. Beck, Department of Civil Engineering, Imperial College of Science and Technology, Imperial College Road, London, England SW7 2BU.
(Received August 30, 1983; revised November 27, 1986; accepted December 15, 1986.) 
